\title{
Analyzing New Neutral Gauge Bosons at the LHC Using Third Generation Fermions
}

by

\author{
Travis A.W. Martin
}

\author{
A thesis submitted to the \\ Faculty of Graduate Studies and Research \\ in partial fulfillment of the requirements \\ for the degree of \\ Master of Science \\ Department of Physics \\ Carleton University \\ Ottawa-Carleton Institute of Physics \\ Ottawa, Canada
}

September 19, 2007

Copyright (c) 2007 Travis A.W. Martin 


$\begin{array}{ll}\begin{array}{l}\text { Library and } \\ \text { Archives Canada }\end{array} & \begin{array}{l}\text { Bibliothèque et } \\ \text { Archives Canada }\end{array} \\ \begin{array}{l}\text { Published Heritage } \\ \text { Branch }\end{array} & \begin{array}{l}\text { Direction du } \\ \text { Patrimoine de l'édition }\end{array} \\ \begin{array}{l}\text { 395 Wellington Street } \\ \text { Ottawa ON K1A ON4 }\end{array} & \begin{array}{l}\text { 395, rue Wellington } \\ \text { Ottawa ON K1A ON4 } \\ \text { Canada }\end{array}\end{array}$

Your file Votre référence ISBN: 978-0-494-33705-9 Our file Notre référence ISBN: 978-0-494-33705-9

NOTICE:

The author has granted a nonexclusive license allowing Library and Archives Canada to reproduce, publish, archive, preserve, conserve, communicate to the public by telecommunication or on the Internet, loan, distribute and sell theses worldwide, for commercial or noncommercial purposes, in microform, paper, electronic and/or any other formats.

The author retains copyright ownership and moral rights in this thesis. Neither the thesis nor substantial extracts from it may be printed or otherwise reproduced without the author's permission.
AVIS:

L'auteur a accordé une licence non exclusive permettant à la Bibliothèque et Archives Canada de reproduire, publier, archiver, sauvegarder, conserver, transmettre au public par télécommunication ou par l'Internet, prêter, distribuer et vendre des thèses partout dans le monde, à des fins commerciales ou autres, sur support microforme, papier, électronique et/ou autres formats.

L'auteur conserve la propriété du droit d'auteur et des droits moraux qui protège cette thèse. $\mathrm{Ni}$ la thèse ni des extraits substantiels de celle-ci ne doivent être imprimés ou autrement reproduits sans son autorisation.
In compliance with the Canadian

Privacy Act some supporting forms may have been removed from this thesis.

While these forms may be included in the document page count, their removal does not represent any loss of content from the thesis.
Conformément à la loi canadienne sur la protection de la vie privée, quelques formulaires secondaires ont été enlevés de cette thèse.

Bien que ces formulaires aient inclus dans la pagination, il n'y aura aucun contenu manquant.

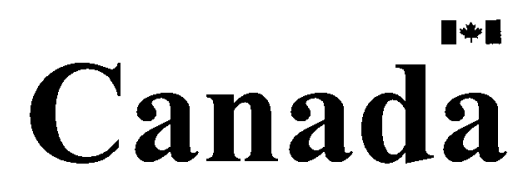




\section{Abstract}

This thesis reports on techniques and the corresponding phenomenology for distinguishing between models in the analysis of new neutral gauge boson production at the LHC. Specifically, third generation final states are reviewed and determined to be effective channels for analysis. Transverse momentum cuts of $p_{T_{f}}>0.3 M_{Z^{\prime}}$ to hadronic final states are employed to reduce QCD backgrounds and distinguish $t$ and $b$ signals. An analysis of the ratio of the $t$ and $b$ cross sections to the $\mu$ cross section, within an invariant mass window of $0.965 M_{Z^{\prime}} \leq M_{f \bar{f}} \leq 1.035 M_{Z^{\prime}}$, is performed. For final state fermion detection efficiencies of $\epsilon_{\mu}=0.96, \epsilon_{t}=0.10$, and $\epsilon_{b}=0.50$, most models considered can be distinguished by at least one standard deviation for $M_{Z^{\prime}}=1500 \mathrm{GeV}$, with $10 \mathrm{fb}^{-1}$ integrated luminosity. Furthermore, the ratio of the $\tau$ to $\mu$ cross sections is analyzed and used to understand models with preferential couplings to third generation particles in the lepton family. Assuming an efficiency of $\epsilon_{\tau}=0.2$, generation universality can be determined up to approximately a $5 \%$ uncertainty for a $1000 \mathrm{GeV} Z^{\prime}$ with $10 \mathrm{fb}^{-1}$ integrated luminosity. 
To my grandfather, Walter Benjamin Sorenson [1913-2004], whose curiosity and determination knew not the shackles that complete vision loss placed upon him, and inspired me to a life of learning. 


\section{Acknowledgements}

It is difficult to list the names of all the people I would like to thank for their support during the course of my M.Sc. program at Carleton University.

First and foremost, I would like to thank my family and friends, who have encouraged and supported me and helped me find the right balance of work and play. Specifically, I would like to thank Wes Burwash for the many late night chats, and for listening to me while I talked out many of the issues I encountered.

I owe much gratitude to my fellow graduate students, Malachi Schram and J.P. Archambault, for their time and assistance. Both of them provided key insights into the world of Experimental High Energy Physics, and assisted me on numerous occasions with technical difficulties encountered.

I would also like to thank Pat Kalyniak, Heather Logan and Bruce Campbell for their assistance on many aspects of theoretical physics and models of BSM, and Gandalf (Juergen Reuter) for introducing me to WHiZard and helping me understand its intricacies.

Last but not least, I owe much gratitude to Steve Godfrey for his supervision and advice, and for standing with me through the process of learning, and changing from an Engineer to a Physicist.

To those whose names do not appear, know that my gratitude is still there. 


\section{Statement of Originality}

Included in this thesis is a review of the author's work resulting from research as an M.Sc. student at Carleton University. All work contained within occurred only at Carleton University. The results within are not yet published, though several papers on the analysis performed are forthcoming.

The results contained within this thesis are entirely the work of the author, under the supervision and guidance of Stephen Godfrey. The review topics within the introductory chapters contain information gathered from other sources, and have been properly referenced and attributed as such. 


\section{Contents}

1 Introduction $\quad 1$

2 The Standard Model of Particle Physics 4

2.1 The Particles and their Interactions . . . . . . . . . . 4

2.1.1 Electroweak Gauge Bosons and their Interactions . . . . . . 5

2.1 .2 Strong Interactions . . . . . . . . . . . . . . . . . . . 9

2.1.3 Issues with the Standard Model . . . . . . . . . . . 10

2.1.4 Beyond the Standard Model . . . . . . . . . . . . . . 13

2.2 Motivation for Examining Neutral Gauge Bosons . . . . . . . . . 15

3 Models Containing Extra Neutral Gauge Bosons 17

3.1 BSM Model Preface . . . . . . . . . . . . . . . . 17

$3.2 Z^{\prime}$ Lagrangian . . . . . . . . . . . . . . . . . . . 18

3.3 Model Descriptions . . . . . . . . . . . . . . . . 19

3.3.1 String Inspired $E_{6}$ Models . . . . . . . . . . . . . 19

vii 
3.3.2 Left Right and Alternate Left Right Symmetric Models . . . . 21

3.3.3 HARV Model . . . . . . . . . . . . . . . . 22

3.3.4 Topcolor Models . . . . . . . . . . . . . . . . . 22

3.3.5 Littlest Higgs Model . . . . . . . . . . . . . . . . . . . . . 24

3.3.6 Simplest Little Higgs Model . . . . . . . . . . . . . . . . . . . 25

3.3.7 Anomaly Free Simple Little Higgs Model . . . . . . . . . . . . 26

3.3.8 3-3-1 Models . . . . . . . . . . . . . . . . . 27

3.3.9 SU(2) $)_{h} \times S U(2)_{l}$ Models $(\mathrm{HLSUM}) \ldots \ldots \ldots$

3.3.10 $U(1)_{h} \times U(1)_{l}$ Models (HLUM) . . . . . . . . . . 29

3.3.11 Benchmark: Sequential Standard Model . . . . . . . . . . . 30

$4 \quad Z^{\prime}$ Observables $\quad 32$

4.1 Existing Observables ..................... 33

4.1.1 Decay Width and Branching Ratios . . . . . . . . . . . 34

4.1.2 Differential Cross Section . . . . . . . . . . . 37

4.1.3 Forward-Backward Asymmetry . . . . . . . . . . . . . 40

4.1.4 Ratios - Rapidity Ratio, Rare Decays and Associated Produc-

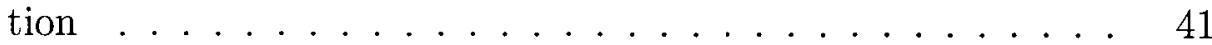

$4.2 Z^{\prime}$ Decays to Heavy Particles . . . . . . . . . . . . . . 45

4.3 Identifying Generation Universality . . . . . . . . . . . . 47

4.4 Parton Distributions, Backgrounds and Efficiencies _........ 49

viii 
4.4.1 Parton Distribution Functions . . . . . . . . . . . . 49

4.4.2 Detector Efficiencies . . . . . . . . . . . . . . 49

4.4.3 Backgrounds and Tagging .............. 53

5 Results $\quad 56$

5.1 Calculation Methods . . . . . . . . . . . . . . . 56

5.2 Discovery potential at the $\mathrm{LHC} \ldots \ldots \ldots \ldots 7$

5.3 Heavy Quark Final States . . . . . . . . . . . . . . . 65

5.3.1 Viability of quark final state measurements . . . . . . 65

5.3.2 Distinguishing between models using $R_{t / \mu}$ and $R_{b / \mu} \ldots \ldots 73$

5.4 Determining Generation Universality in $Z^{\prime} \ldots \ldots$. . . . . . 87

5.4.1 Lepton channel . . . . . . . . . . . . . . . 87

$\begin{array}{lll}6 & \text { Summary } & 100\end{array}$

$\begin{array}{ll}\text { References } & 102\end{array}$ 


\section{List of Tables}

2.1 Standard Model Particles and their Properties . . . . . . . . . . . 6

3.1 Couplings of SM Fermions in the $E_{6} \psi$ and $\chi$ Models . . . . . . 20

3.2 Couplings of SM Fermions under Topcolor symmetry . . . . . . . . . 24

3.3 Couplings of SM Fermions under Simplest Little Higgs symmetry . 26

3.4 Couplings of SM Fermions under Anomaly Free Simple Little Higgs symmetry . . . . . . . . . . . . . . . . . 27

3.5 Couplings of the SM Fermions under the 3-3-1 Model symmetry . . . 28

3.6 Couplings of the SM Fermions in the Sequential Standard Model . . . 31

4.1 Ratio of $\Gamma_{Z^{\prime}} / M_{Z^{\prime}}$ for each considered model at $M_{Z^{\prime}}=1$ and $3 \mathrm{TeV} . \quad 36$

4.2 Detection efficiencies for $e \& \mu$ leptons, and $c$ jets for ATLAS. . . . . 53

5.1 Ratio of $\mathrm{S} / \sqrt{\mathrm{B}}$ at detection luminosity for $M_{Z^{\prime}}=1,2,3 \mathrm{TeV} \ldots \ldots 1$

5.2 Ratio of $\mathrm{S} / \sqrt{\mathrm{S}+\mathrm{B}}$ for $b \bar{b}$ and $t \bar{t}$ pairs for $10 \mathrm{fb}^{-1}$ integrated luminosity and optimistic efficiencies. . . . . . . . . . . . . . 71 
5.3 Ratio of $\mathrm{S} / \sqrt{\mathrm{S}+\mathrm{B}}$ for $b \bar{b}$ and $t \bar{t}$ pairs for $10 \mathrm{fb}^{-1}$ integrated luminosity and pessimistic efficiencies. . . . . . . . . . . . 72

$5.4 R_{\tau / \mu}$ for a 1,2 and $3 \mathrm{TeV} Z^{\prime}$ with $\epsilon_{\tau}=0.20 \ldots \ldots \ldots$

$5.5 R_{\tau / \mu}$ for a 1,2 and $3 \mathrm{TeV} Z^{\prime}$ with $\epsilon_{\tau}=0.01 \ldots \ldots 90$ 


\section{List of Figures}

2.1 Electroweak vertices involving the interaction of fermions via a gauge boson ............................. 8

2.2 Electroweak vertices involving the interactions of only gauge bosons . 8

2.3 QCD vertices in the Standard Model . . . . . . . . . . . . 10

4.1 Drell-Yan diagram for $p p$ collisions at LHC, including a $Z^{\prime} \ldots \ldots$

4.2 $G_{q}^{ \pm}(y, M)$ vs rapidity at energy scale $M_{q \bar{q}}=1 \mathrm{TeV}$ for $u, d, s, c$, and $b$

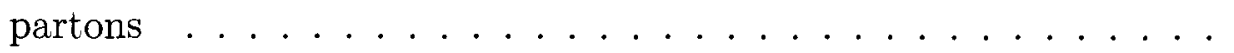

4.3 Heavy quark backgrounds, assuming distinguishability of final state

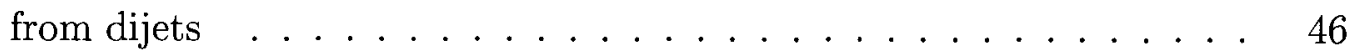

4.4 QCD diagrams involved in dijet backgrounds that do not result in heavy quark final states . . . . . . . . . . . . . . 46

5.1 Invariant Mass Distribution for $M_{Z^{\prime}}=1,2$ and $3 \mathrm{TeV}$ for a representative set of models, in the $\mu^{+} \mu^{-}$channel . . . . . . . . . . 58

5.2 Luminosity required for discovery of a $Z^{\prime}$ in the dimuon channel (Primarily Gen. Univ. models) . . . . . . . . . . . . . . . . 
5.3 Luminosity required for discovery of a $Z^{\prime}$ in dimuon channel (Primarily non-Gen. Univ. models) . . . . . . . . . . . . . . . . 60

5.4 Invariant Mass Distribution for $M_{Z^{\prime}}=1,2$ and $3 \mathrm{TeV}$ in the $\tau^{+} \tau^{-}$ channel, for a representative set of models. . . . . . . . . . . 62

5.5 Luminosity required for discovery of a $Z^{\prime}$ in the di-tau channel (Primarily Gen. Univ. models) $\ldots \ldots \ldots \ldots \ldots \ldots$

5.6 Luminosity required for discovery of a $Z^{\prime}$ in di-tau channel (Primarily non-Gen. Univ. models) . . . . . . . . . . . . . . . . . . . .

5.7 Invariant mass distribution for production of $t \bar{t}$ pairs with no cuts for a representative set of models. $\ldots \ldots \ldots \ldots \ldots \ldots$

5.8 Invariant mass distribution for production of $t \bar{t}$ pairs with $p_{T}>0.3 M_{Z^{\prime}}$ for a representative set of models. . . . . . . . . . . . . . .

5.9 Invariant mass distribution for production of $b \bar{b}$ pairs with no cuts for a representative set of models. . . . . . . . . . . . . . .

5.10 Invariant mass distribution for production of $b \bar{b}$ pairs with $p_{T}>0.3 M_{Z^{\prime}}$ for a representative set of models.

5.11 Plots of $1 \sigma$ uncertainty bounds for $R_{b / \mu}$ vs $R_{t / \mu}$ for $M_{Z^{\prime}}=2000 \mathrm{GeV}$ at pessimistic efficiencies, showing effect of increasing luminosity on uncertainty bounds $(1) \ldots \ldots \ldots \ldots \ldots \ldots$

5.12 Plots of $1 \sigma$ uncertainty bounds for $R_{b / \mu}$ vs $R_{t / \mu}$ for $M_{Z^{\prime}}=2000 \mathrm{GeV}$ at pessimistic efficiencies, showing effect of increasing luminosity on uncertainty bounds $(2) \ldots \ldots \ldots \ldots \ldots \ldots$ 
5.13 Plots of $1 \sigma$ uncertainty bounds for $R_{b / \mu}$ vs $R_{t / \mu}$ for $M_{Z^{\prime}}=2000 \mathrm{GeV}$ at pessimistic efficiencies, showing effect of increasing luminosity on uncertainty bounds $(3) \ldots \ldots \ldots \ldots \ldots$

5.14 Plots of $1 \sigma$ uncertainty bounds for $R_{b / \mu}$ vs $R_{t / \mu}$ for $M_{Z^{\prime}}=2000 \mathrm{GeV}$ at optimistic efficiencies, showing effect of increasing luminosity on uncertainty bounds $(1) \ldots \ldots \ldots \ldots \ldots$

5.15 Plots of $1 \sigma$ uncertainty bounds for $R_{b / \mu}$ vs $R_{t / \mu}$ for $M_{Z^{\prime}}=2000 \mathrm{GeV}$ at optimistic efficiencies, showing effect of increasing luminosity on uncertainty bounds $(2) \ldots \ldots \ldots \ldots \ldots$

5.16 Plots of $1 \sigma$ uncertainty bounds for $R_{b / \mu}$ vs $R_{t / \mu}$ for $M_{Z^{\prime}}=2000 \mathrm{GeV}$ at optimistic efficiencies, showing effect of increasing luminosity on uncertainty bounds $(3) \ldots \ldots \ldots \ldots \ldots \ldots \ldots$

5.17 Plots of $1 \sigma$ uncertainty bounds for $R_{b / \mu}$ vs $R_{t / \mu}$ for integrated luminosity $\mathrm{L}=100 \mathrm{fb}^{-1}$, at pessimistic efficiencies, showing effect of increasing mass on uncertainty bounds $(1) \ldots \ldots \ldots \ldots$

5.18 Plots of $1 \sigma$ uncertainty bounds for $R_{b / \mu}$ vs $R_{t / \mu}$ for integrated luminosity $\mathrm{L}=100 \mathrm{fb}^{-1}$, at pessimistic efficiencies, showing effect of increasing mass on uncertainty bounds $(2) \ldots \ldots \ldots \ldots$

5.19 Plots of $1 \sigma$ uncertainty bounds for $R_{b / \mu}$ vs $R_{t / \mu}$ for integrated luminosity $\mathrm{L}=100 \mathrm{fb}^{-1}$, at pessimistic efficiencies, showing effect of increasing mass on uncertainty bounds $(3) \ldots \ldots \ldots \ldots \ldots$

5.20 Plots of $1 \sigma$ uncertainty bounds for $R_{b / \mu}$ vs $R_{t / \mu}$ for integrated luminosity $\mathrm{L}=100 \mathrm{fb}^{-1}$, at optimistic efficiencies, showing effect of increasing mass on uncertainty bounds $(1) \ldots \ldots \ldots \ldots \ldots$ 
5.21 Plots of $1 \sigma$ uncertainty bounds for $R_{b / \mu}$ vs $R_{t / \mu}$ for integrated luminosity $\mathrm{L}=100 \mathrm{fb}^{-1}$, at optimistic efficiencies, showing effect of increasing mass on uncertainty bounds $(2) \ldots \ldots \ldots$

5.22 Plots of $1 \sigma$ uncertainty bounds for $R_{b / \mu}$ vs $R_{t / \mu}$ for integrated luminosity $\mathrm{L}=100 \mathrm{fb}^{-1}$, at optimistic efficiencies, showing effect of increasing mass on uncertainty bounds $(3) \ldots \ldots \ldots$

5.23 Plot of $R_{\tau / \mu}$ for each model at $10 \mathrm{fb}^{-1}$ integrated luminosity and pessimistic efficiencies. (Range selected to highlight generation universal

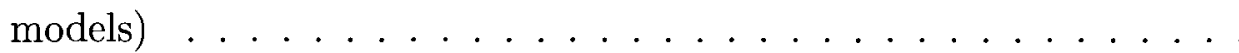

5.24 Plot of $R_{\tau / \mu}$ for each model at $10 \mathrm{fb}^{-1}$ integrated luminosity and pessimistic efficiencies. (Range selected to highlight non-generation universal models $\ldots \ldots \ldots \ldots \ldots \ldots \ldots$

5.25 Plot of $R_{\tau / \mu}$ for each model at $10 \mathrm{fb}^{-1}$ integrated luminosity and optimistic efficiencies. (Range selected to highlight generation universal

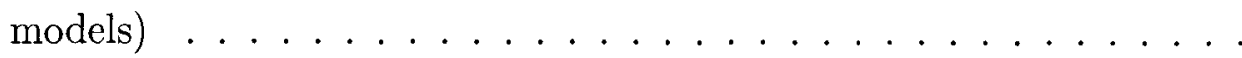

5.26 Plot of $R_{\tau / \mu}$ for each model at $10 \mathrm{fb}^{-1}$ integrated luminosity and optimistic efficiencies. (Range selected to highlight non-generation univer-

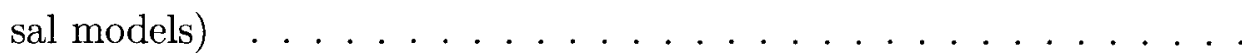

5.27 Plot of $R_{\tau / \mu}$ for each model at $300 \mathrm{fb}^{-1}$ integrated luminosity and pessimistic efficiencies. (Range selected to highlight generation universal

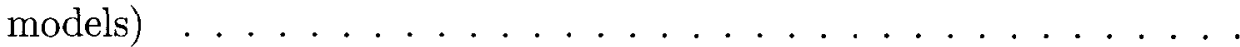

5.28 Plot of $R_{\tau / \mu}$ for each model at $300 \mathrm{fb}^{-1}$ integrated luminosity and pessimistic efficiencies. (Range selected to highlight non-generation universal models $\ldots \ldots \ldots \ldots \ldots \ldots \ldots$ 
5.29 Plot of $R_{\tau / \mu}$ for each model at $300 \mathrm{fb}^{-1}$ integrated luminosity and optimistic efficiencies. (Range selected to highlight generation universal

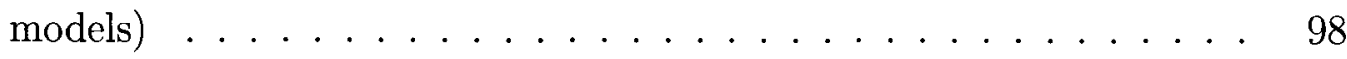

5.30 Plot of $R_{\tau / \mu}$ for each model at $300 \mathrm{fb}^{-1}$ integrated luminosity and optimistic efficiencies. (Range selected to highlight non-generation universal models $) \ldots \ldots \ldots \ldots \ldots \ldots \ldots$ 


\section{Chapter 1}

\section{Introduction}

The realm of high energy physics has reached a threshold defined by the electroweak scale of $\mathcal{O}(\mathrm{TeV})$. The recent advances in the field by the Tevatron facility have confirmed the existence of the final Standard Model fermion - the top quark ${ }^{1}$ $\left(m_{t}=174 \mathrm{GeV}\right)$ - by colliding protons and antiprotons at centre of mass energies of $1.96 \mathrm{TeV}$. But the top quark remains the highest mass fundamental particle discovered and the theorized Higgs boson still eludes experimentalists.

The current, combined theoretical knowledge that describes high energy experimental results can be summarized in what is known as the Standard Model of Particle Physics (SM). Experiments have confirmed many aspects of this model to very high precision and the SM stands as a coherent descriptor of how subatomic particles interact. However, the model is incomplete; the Higgs boson (the theoretical particle in the SM responsible for mass) has not been found, the nature of the neutrino is in question, and astrophysical measurements suggest the existence of some form of dark matter - see Section 2.1.3. 
Over the past three decades, new theories have been developed to explain and to fill the gaps in the SM, with each attempt incorporated into a new "model". While some of these models are compatible with each other, and can be simultaneously realized, others are mutually exclusive. In general, all of these models are collectively incorporated under the name "Beyond the Standard Model" (BSM). An overview of a number of models of BSM are included in Chapter 3.

The Tevatron is currently the highest energy particle collider, and has been examining fundamental interactions of particles since it was completed in 1983. It has gone through several upgrades to improve results throughout the years and is currently working on Run II. The two collider experiments at the Tevatron, D0 and CDF, work competitively to both search for new physics and to make more precise measurements of the properties of known particles. In most cases, they study the same phenomena and the data can be compared and combined for a better understanding of the processes involved, as was the case with the top quark measurements.

A new particle collider is currently under the final stages of construction at CERN, Geneva, Switzerland, called the Large Hadron Collider (LHC). This new facility, along with the primary detector experiments, ATLAS and CMS, will examine proton-proton collisions at a centre of mass energy of $14 \mathrm{TeV}$. These will be the highest energy artificially created collisions to date, and are intended to explore many of the issues with the SM that remain unresolved from the Tevatron. It is expected that initial data will be collected in Spring 2008 at low luminosities, with the luminosity increasing in the future. A high precision $e^{+} e^{-}$collider, the International Linear Collider (ILC), is being planned to follow and act as a complement ${ }^{2}$ to the LHC.

With the large increase in energy at the LHC over the Tevatron, many physicists expect that the LHC will produce a variety of new particles that have not yet 
been discovered. These possibilities include new bosons and fermions, as well as exotic new particles. In order to differentiate between the possible models that can generate these new particles, new analysis techniques specific to the LHC have been designed to understand the phenomena. ${ }^{3-5}$ These techniques are sensitive to the couplings of typically one or two other particles and highlight the differences between models to allow a clear distinction between them.

Common to most theories of BSM is the existence of another neutral gauge boson, commonly called a $Z^{\prime}$, and it is this particle that is the focus of this thesis. Chapter 4 reviews several techniques covered in the literature useful in distinguishing between different models (models discussed in Chapter 3 ) of new neutral gauge bosons, and further suggests new techniques. Specifically, the new techniques are sensitive to the couplings to third generation fermions and produce distinctive results for models that have generation dependent couplings, such as Topcolor models.

Identification of third generation final states in the ATLAS and CMS detectors remains a significant challenge to experimentalists and to accurate measurement with the techniques covered within this thesis. Several scenarios of feasible tagging efficiencies are considered in this thesis in Section 4.4, and an analysis of the corresponding luminosities required to distinguish between models is included in Chapter 5.

Results and calculations are presented in Chapter 5, including expected luminosities for the discovery of a $Z^{\prime}$ and the phenomenology for the proposed observables discussed in Chapter 4. A summary follows in Chapter 6. 


\section{Chapter 2}

\section{The Standard Model of Particle}

\section{Physics}

\subsection{The Particles and their Interactions}

There exist two types of fundamental particles within the Standard Model $(\mathrm{SM})^{6}$ fermions and bosons. Fermions, particles with half integer multiples of spin, are known to correspond to "matter", while bosons, particles with integer multiples of spin, are the "force" particles. What was classically understood as a "force," however, has led the way to the understanding that these particles simply mediate the interactions between all particles - both fermions and bosons.

The SM also contains some unusual features that arise from experimental evidence. For example, there does not appear to be a right handed neutrino, as witnessed in beta decay. Additionally, each of the fermion types occur in three generations, with increasing masses for each generation. A listing of properties and the hierarchy of the 
SM particles are listed in Table 2.1, below.

In terms of group symmetry, the Standard Model interactions are described by an $S U(3)_{c} \times S U(2)_{L} \times U(1)_{Y} \cdot{ }^{7}$ The $S U(3)_{c}$ describes the interactions of particles that contain color charge, while the $S U(2)_{L} \times U(1)_{Y}$ describes the unified interactions of particles with electric charge and weak hypercharge. The gauge bosons $\left(Z, W^{ \pm}, \gamma, g\right)$ then occur because we demand local gauge invariance under these symmetry transformations.

\subsubsection{Electroweak Gauge Bosons and their Interactions}

In the unified Electroweak (EW) theory, developed by Glashow, ${ }^{8}$ Weinberg ${ }^{9}$ and Salam $^{10}$ (GWS) between 1961 to 1968, Electroweak interactions are based on the $S U(2)_{L} \times U(1)_{Y}$ symmetry group. The SM fermions can be classified into chiral components, with left handed components that transform as doublets under the $S U(2)$ and right handed components that transform as singlets, as below:

$$
\begin{array}{ll}
q_{L}=\left(\begin{array}{c}
u_{L}^{i} \\
d_{L}^{i}
\end{array}\right) & \ell_{L}=\left(\begin{array}{c}
\nu_{L}^{i} \\
e_{L}^{i}
\end{array}\right) \\
q_{R}=u_{R}^{i}, d_{R}^{i} & \ell_{R}=e_{R}^{i}
\end{array}
$$

This gauge group includes four gauge bosons, $\bar{W}_{\mu}=\left(W_{\mu}^{1}, W_{\mu}^{2}, W_{\mu}^{3}\right)$ for the $S U(2)$ and $B_{\mu}$ for the $U(1)$, with corresponding coupling strengths $g$ and $g^{\prime}$, respectively. This symmetry state is then spontaneously broken to a vacuum state by the Higgs mechanism, and the gauge bosons take the form of the interaction eigenstates with specific masses. The resulting EW Lagrangian ${ }^{7}$ governing the interactions of 
Table 2.1: A listing of the fundamental particles included in the Standard Model, ${ }^{7}$ including their hierarchy and some of their properties.

$\dagger$ This is an assumed value. Experiment does not preclude a mass of less than a few $\mathrm{MeV}$.

\begin{tabular}{|c|c|c|c|c|}
\hline Type & Family & Particle & Mass $(\mathrm{MeV})$ & Charge \\
\hline \multirow{12}{*}{ fermion } & \multirow{3}{*}{$\begin{array}{l}\text { u-type } \\
\text { quark }\end{array}$} & up $(u)$ & $1.5-3.0$ & $+2 / 3$ \\
\hline & & charm $(c)$ & $1250 \pm 90$ & $+2 / 3$ \\
\hline & & top $(t)$ & $174200 \pm 2000$ & $+2 / 3$ \\
\hline & \multirow{3}{*}{$\begin{array}{l}\text { d-type } \\
\text { quark }\end{array}$} & down $(d)$ & $3.0-7.0$ & $-1 / 3$ \\
\hline & & strange $(s)$ & $95 \pm 25$ & $-1 / 3$ \\
\hline & & bottom $(b)$ & $4200 \pm 70$ & $-1 / 3$ \\
\hline & \multirow{3}{*}{$\begin{array}{l}\text { e-type } \\
\text { lepton }\end{array}$} & electron $(e)$ & 0.511 & -1 \\
\hline & & muon $(\mu)$ & 105.66 & -1 \\
\hline & & $\operatorname{tau}(\tau)$ & $1776.99_{-0.26}^{+0.29}$ & -1 \\
\hline & \multirow{3}{*}{$\begin{array}{l}\nu \text {-type } \\
\text { lepton }\end{array}$} & electron neutrino $\left(\nu_{e}\right)$ & $<2 \times 10^{-6}$ & 0 \\
\hline & & muon neutrino $\left(\nu_{\mu}\right)$ & $<0.19$ & 0 \\
\hline & & tau neutrino $\left(\nu_{\tau}\right)$ & $<18.2$ & 0 \\
\hline Type & Force & Particle & $\operatorname{Mass}(\mathrm{GeV})$ & Charge \\
\hline \multirow{5}{*}{ boson } & strong & gluon $(g)$ & $0^{\dagger}$ & 0 \\
\hline & electric & photon $(\gamma)$ & $<6 \times 10^{-26}$ & 0 \\
\hline & weak & Z-boson $(Z)$ & $91.1876 \pm 0.0021$ & 0 \\
\hline & weak & W-boson $\left(W^{ \pm}\right)$ & $80.403 \pm 0.029$ & \pm 1 \\
\hline & $\mathrm{n} / \mathrm{a}$ & Higgs boson $(H)$ & $>114.4$ & 0 \\
\hline
\end{tabular}


fermions via EW gauge bosons is given by: ${ }^{7}$

$$
\begin{aligned}
& \mathcal{L}_{E W}= \sum_{i} \bar{\psi}_{i}\left(i \gamma^{\mu} \partial_{\mu}-m_{i}\right) \psi_{i}+\mathcal{L}_{N C}+\mathcal{L}_{C C} \\
& \mathcal{L}_{N C}=-\sum_{j} \bar{\psi}_{j} \gamma^{\mu} A_{\mu}\left[g T_{j}^{3} \mathrm{~s}_{W}+g^{\prime} y_{j} \mathrm{c}_{W}\right] \psi_{j} \\
& \quad-\sum_{j} \bar{\psi}_{j} \gamma^{\mu} Z_{\mu}\left[g T_{j}^{3} \mathrm{c}_{W}-g^{\prime} y_{j} \mathrm{~s}_{W}\right] \psi_{j} \\
& \mathcal{L}_{C C}=-\sum_{j} \frac{g}{\sqrt{2}} \bar{\psi}_{j} \gamma^{\mu}\left(1-\gamma^{5}\right)\left[T^{+} W_{\mu}^{+}+T^{-} W_{\mu}^{-}\right] \psi_{i}
\end{aligned}
$$

Where $y_{j}=q_{j}-T_{j}^{3}$ is the weak hypercharge for fermion flavour $j$, in terms of electromagnetic charge $q_{j}$ and third component of isospin $T_{j}^{3} \cdot T^{ \pm}$are the isospin raising/lowering operators, and $g \sin \theta_{W}=g^{\prime} \cos \theta_{W}=e$ are the coupling strengths. The angle $\theta_{W}$ is the weak angle, given by $\sin ^{2} \theta_{W} \equiv 1-M_{W}^{2} / M_{Z}^{2}=0.23122(\overline{\mathrm{MS}}$ scheme $)$. The fields denoted by $\psi_{j}$ represent the fermions listed previously, and the Lagrangian subscripts $\mathrm{NC}$ and $\mathrm{CC}$ denote the neutral current and charged current respectively.

The interaction eigenstates denoted by the massless photon, $A_{\mu}$, and the massive $Z$ and $W$ bosons, $Z_{\mu}$ and $W_{\mu}^{ \pm}$, are related to the fundamental $\bar{W}_{\mu}$ and $B_{\mu}$ fields by the following relations:

$$
\begin{aligned}
A_{\mu} & \equiv B_{\mu} \cos \theta_{W}+W^{3} \sin \theta_{W} \\
Z_{\mu} & \equiv-B_{\mu} \sin \theta_{W}+W^{3} \cos \theta_{W} \\
W_{\mu}^{ \pm} & \equiv\left(W_{\mu}^{1} \mp i W^{2}\right)
\end{aligned}
$$

Figures 2.1 and 2.2 show the Feynman diagrams representing the ten threeand four-point interaction vertices involved in Electroweak Theory. ${ }^{6}$ These describe the interactions of fermions with the gauge bosons, and the self interactions of the gauge bosons. Any purely Electroweak interaction can be described schematically via combinations of these vertices. 

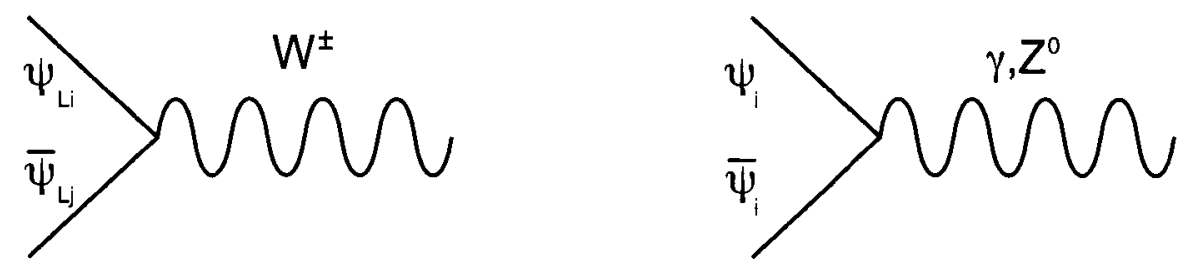

Figure 2.1: Feynman diagrams involving the interaction of fermions with Electroweak gauge bosons. $\psi_{i}$ represents any chiral fermion. $\psi_{L i, j}$ represent any complementary left handed chiral fields - either $e_{L} \nu_{L}$ or $u_{L} d_{L}$.
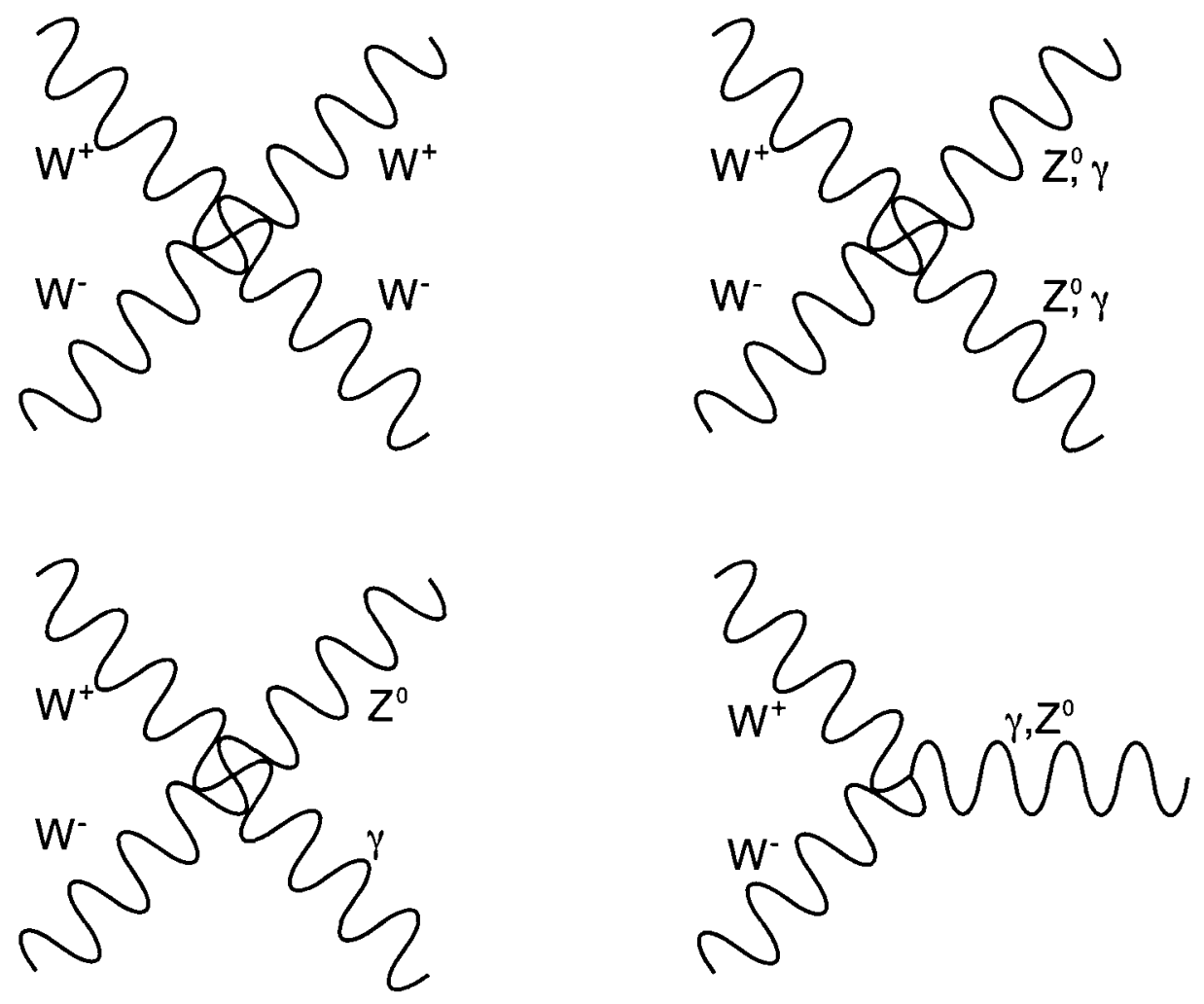

Figure 2.2: Three and four point vertices involving only the gauge bosons $-W^{ \pm}, \gamma$ and $Z^{0}$. 


\subsubsection{Strong Interactions}

The gauge field theory describing the strong interaction is known as Quantum Chromodynamics (QCD $)^{6,7}$ and describes interactions between particles with color charge. Color charge comes in three varieties for quarks (denoted r, g or b) and three anticolor varieties for anti-quarks $(\bar{r}, \bar{g}$, or $\bar{b})$. The mediator of the interaction, the gluon, carries both color and anti-color charges. Curiously, the strong force has a property known as "asymptotic freedom", where the strong interaction can be treated perturbatively only at high energies/short distances. At low energies/large distances, the strong coupling becomes large and perturbation theory breaks down. These low energy effects are not obvious in nature, as bound states of quarks and gluons form color neutral singlets.

The self interactions of the gluons represent a major component of QCD interactions at the LHC and are included in the QCD Lagrangian presented in Equation (2.7), below.

$$
\begin{aligned}
\mathcal{L}_{Q C D} & =-\frac{1}{4} F_{\mu \nu}^{(a)} F^{(a) \mu \nu}+i \sum_{q} \bar{\psi}_{q}^{i} \gamma^{\mu}\left(D_{\mu}\right)_{i j} \psi_{q}^{j}-\sum_{q} m_{q} \bar{\psi}_{q}^{i} \psi_{q i} \\
F_{\mu \nu}^{(a)} & =\partial_{\mu} A_{\nu}^{a}-\partial_{\nu} A_{\mu}^{a}-g_{s} f_{a b c} A_{\mu}^{b} A_{\nu}^{c} \\
\left(D_{\mu}\right)_{i j} & =\delta_{i j} \partial_{\mu}+i g_{s} \sum_{a} \frac{\lambda_{i j}^{a}}{2} A_{\mu}^{a}
\end{aligned}
$$

In this form, $\psi_{q}^{i}$ are the quark fields for color $i$ and flavour $q, A_{\mu}^{a}$ are the gluon fields, $g_{s}$ is the strong coupling strength, and $f_{a b c}$ are the $S U(3)$ structure constants. The $3 \times 3$ Gell-Mann matrices, $\lambda_{i j}^{a}$, are the generators of the $S U(3)$ transformations. The resulting QCD vertex diagrams are shown in Figure 2.3.

While there are six flavours of quarks - down, up, strange, charm, bottom, top - each comes in each of the three colors, totaling 18 quarks. Additionally, the 

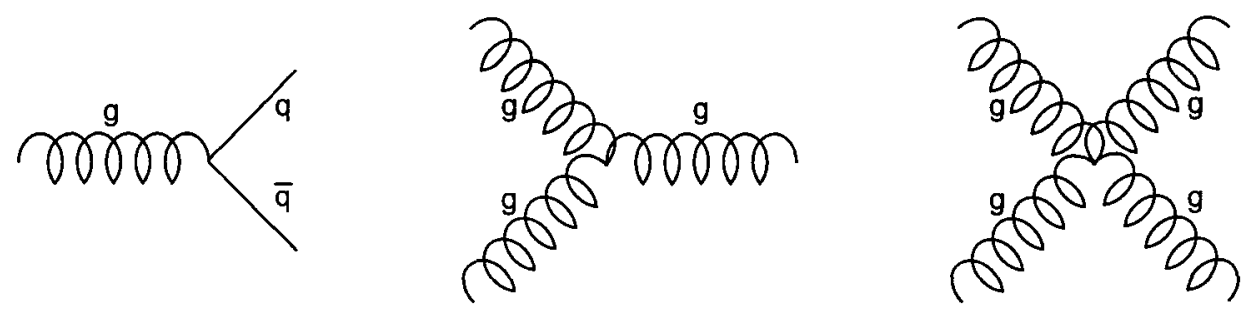

Figure 2.3: Particle interaction vertices arising from the strong interaction, including quark interactions and gluon three and four point vertices.

$S U(3)_{c}$ symmetry results in eight states for gluons, having combinations of color and anti-color.

In this thesis, the inclusion of QCD interactions is limited to the calculation of backgrounds to EW processes involving quark final states. This overview is provided as a general insight into the differences between the two processes (QCD and EW).

\subsubsection{Issues with the Standard Model}

The prediction and later experimental confirmation of the $W, Z$ and top quark existence and their masses was a strong confirmation of the Standard Model. For approximately the past 30 years, the SM has formed the basis of our understanding of particle physics and agrees with experimental data to incredible precision. However, the SM is not complete and does not explain some recent measurements and observations in nature. Many of these issues are listed below.

\section{Electroweak Symmetry Breaking}

What is the exact nature of the mechanism of Electroweak Symmetry Breaking, which is the mechanism that gives mass to particles?

Experiments to find the Higgs Boson have, as yet, been unable to find the 
particle. There are both lower and upper constraints on the mass of the SM Higgs from experiment and EW precision data. The lower bound from experiment is $114.4 \mathrm{GeV}^{7}$ from the LEP2 search in the $e^{+} e^{-} \rightarrow H^{0} Z$ process. The upper bound is placed by fits to $m_{t}, m_{W}$ and other measured data, and is generally within the range of $150-$ $200 \mathrm{GeV} .^{7}$ There is also an upper limit of $710 \mathrm{GeV}^{11}$ as the mass where perturbation theory breaks down.

\section{Hierarchy Problem}

Why does gravity apparently become strong at the Planck scale, which is 16 orders of magnitude larger than the Electroweak scale?

The Planck scale is the term given to the energy scale at which the gravitational interactions become significant, and is approximately $10^{19} \mathrm{GeV}$. However, the electroweak scale, taken as approximately $1 \mathrm{TeV}$, is many orders of magnitude smaller than the Planck scale. This difference in magnitudes does not have an explanation in the SM.

\section{Dark Matter}

What is the particulate nature behind the observation of the excess of mass/gravity in large cosmological structures?

Cosmologists have witnessed strong evidence for the existence of "dark matter" - a weakly interacting particle of unknown but non-zero mass - through observations of gravitational lensing, and of greater rotational speeds in galactic clusters than visible mass indicates. In fact, the amount of mass accounted for by particles that interact electromagnetically is far below that of observations of total mass. Measurements of 
the matter density of celestial bodies suggest that approximately $3-4 \%$ of the energy density in the Universe appears to be visible matter, while about $22 \%$ appears to be dark matter, and the rest appears to be what is known as Dark Energy. One possible SM explanation, neutrino masses, has already been shown not to sufficiently account for the excess mass. This discrepancy cannot be accounted for in the SM.

\section{Neutrino Oscillations}

Are neutrinos Majorana particles? Do neutrinos have mass? If so, what is their mass?

The SNO experiments in Sudbury, ${ }^{12}$ along with the Kamiokande ${ }^{13}$ experiments in Japan, have shown that neutrinos have mass, albeit small, and are observed to oscillate between flavours in the transition from the Sun to Earth. This property can be understood if neutrinos have mass, however the SM assumes this is not the case. The nature of neutrinos is still not determined: neutrinos can either be Dirac fermions, as the other particles of the SM are, or they can be Majorana particles, where the right handed component is the conjugate of the left.

\section{Baryon Asymmetry}

Why is the universe apparently filled with matter rather than an equal amount of matter and anti-matter?

Since particle/anti-particle annihilations are not commonly observed in nature, we know that the vast majority of particles in the universe are of a similar kind - "matter" as opposed to "anti-matter". However, in big-bang cosmology, the universe should have started out with equal amounts of particles as anti-particles. The resulting difference is termed the baryon asymmetry, and is currently not accounted 
for in the SM.

Gravity

What is the particle nature of gravity?

Gravity is theorized to be mediated by a spin 2 particle known as the graviton. However, due to the scale at which gravity becomes significant, the Planck scale, this cannot be probed with current technology. Gravity is not included in the SM, but it is considered to be one of the fundamental forces.

\subsubsection{Beyond the Standard Model}

For the reasons stated above, high energy physicists believe that the SM is incomplete; there must be some mechanism or theory other than the SM that will explain these, and other, unknowns. Following in the footsteps of Glashow, Weinberg and Salam, various models have been developed to try to explain one or more of the issues with the SM. The collection of all these theories are incorporated into the descriptive term, "Beyond the Standard Model" (BSM). The LHC at CERN was developed primarily

to understand the method of Electroweak Symmetry Breaking (EWSB), but also to search for indications of BSM and to examine high energy properties of the SM.

Some models attempt to combine all the three SM interactions into a single theory - unifying EW dynamics with QCD. These models are dubbed Grand Unified Theories (GUTs) and it is possible that the group structure of these GUTs may be examined at the LHC. Other models simply look at possible groups that break to the SM QCD and EW interactions below some scale. As a result, many of the models discussed are not mutually exclusive. 
In general, models that fit within these criteria include:

- Left Right Symmetric and Alternate Left Right Symmetric Model ${ }^{14-19}$

- $E_{6}$ Models (such as $\psi, \chi$, and $\left.\eta\right)^{20,21}$

- 3-3-1 Models 22,23

- Supersymmetry and variants

- Technicolor/Topcolor ${ }^{24-26}$

- Little Higgs Models and variants ${ }^{27-33}$

- Ununified Standard Model ${ }^{34,35}$

- Models with a $U(1)_{h} \times U(1)_{l}$ structure $\mathrm{s}^{26,36-39}$

- Models with an $S U(2)_{h} \times S U(2)_{l}$ structure $^{40-45}$

Other models are dubbed Theories of Everything (ToE), and go even further than GUTs by including gravitational interactions. While not the subject of the analysis of this thesis, these models include:

- String Theory

- M-Theory

- Supergravity

- Macroscopic Extra Dimension Models (ADD, UED, RS) 


\subsection{Motivation for Examining Neutral Gauge Bosons}

One item common to most models of BSM is the prediction of the existence of other neutral gauge bosons, commonly called $Z^{\prime}$ s. In these models, the SM Electroweak group results from the breaking of a more fundamental representation below some energy scale by a model-dependent mechanism. Should such a particle exist with a kinematically accessible mass, its discovery is expected to be one of the first at the LHC.

However, the subsequent determination of the nature of the particle will be more difficult. Primarily, the LHC will be able to narrow down the list of models that can describe the nature of the $Z^{\prime}$. Assuming a low mass $Z^{\prime}$, and high tagging efficiencies, this may uniquely distinguish the nature of the boson, but, for a high mass $Z^{\prime}$ and low tagging efficiencies, the situation may be more pessimistic. A further examination of the individual couplings themselves will require a high precision $e^{+} e^{-}$ collider. The benefit of such a collider is that it can single out initial and final states to uniquely determine the nature of the couplings of the $Z^{\prime}$ to the SM fermions.

For these reasons, this thesis examines the phenomenology of the extra neutral Electroweak sector in order to contribute to the literature on methods for distinguishing between models. Depending on the nature of BSM that is encountered, physicists may also encounter other new neutral particles, such as Kaluza-Klein photon and gluon excitations. For a recent review of distinguishing excitations, please see the paper by Rizzo ${ }^{46}$ and the references within. These are not within the scope of this thesis, however, but do present an extra challenge (i.e.: distinguishing a photon excitation from a $Z^{\prime}$ excitation) for experimentalists beyond simply distinguishing between 
models.

It should be noted that there is no guarantee of a discovery, as current experimental limits place a lower mass $\operatorname{limit}^{7,47}$ on an extra neutral gauge boson at $>600 \mathrm{GeV}$ for some models, to $>800 \mathrm{GeV}$ for other models. As the mass increases, the likelihood of discovery decreases and, more importantly, the ability to distinguish between models decreases, as will be shown.

Erler and Langacker ${ }^{48}$ suggest that there is evidence for an extra neutral gauge boson in precision data collected at LEP, due to data on missing invisible width in $Z$ decays, and effects on weak charge in atomic parity violation. They assumed the existence of a fairly arbitrary GUT type $Z^{\prime}$ and claim an excellent fit between the data and theory. They find evidence for a $Z^{\prime}$ mass of approximately $812_{-152}^{+339} \mathrm{GeV}$ for a $Z_{E_{6}}^{\prime}\left(\chi\right.$ model) or $782_{-241}^{+362} \mathrm{GeV}$ for a $Z_{L R S M}^{\prime}$ (models discussed in Chapter 3). This is not definitive, however, and the status of the $Z^{\prime}$ is still uncertain.

Since there are no significant current plans to build a more powerful collider than the LHC, the LHC should remain the highest energy collider for some time. Should a $Z^{\prime}$ be discovered within the near future, it will likely be discovered at the LHC. 


\section{Chapter 3}

\section{Models Containing Extra Neutral}

\section{Gauge Bosons}

\subsection{BSM Model Preface}

A number of theoretical models are described in the following sections, and are defined in more detail in the references listed. In general, this thesis examines the phenomenology of these models, rather than the models themselves. This is not a complete list, though it is extensive. In addition, the full range of possible values of free parameters within the models are not the focus of this thesis, for brevity and clarity. 


\section{2 $Z^{\prime}$ Lagrangian}

The models of BSM covered in this thesis will be limited to only those that contain extra neutral gauge bosons, while backgrounds covered will be limited to only the dominant SM processes. Other processes that could produce similar final states, including Higgs exchange, are neglected due to expectations that the cross section contribution will be small at the mass of the $Z^{\prime}$.

A general Lagrangian describing a single $Z^{\prime}$, including $Z$ and $Z^{\prime}$ mixing, is given in Equation (3.1). ${ }^{7}$ The left and right handed couplings for each fermion are listed in the following subsections for each model.

$$
\begin{aligned}
\mathcal{L}_{F}=- & \frac{1}{4} F_{\mu \nu}^{\prime} F^{\prime \mu \nu}-\frac{\sin \chi}{2} F_{\mu \nu}^{\prime} F^{\mu \nu}+M_{Z^{\prime}}^{2} Z_{\mu}^{\prime} Z^{\prime \mu}+\delta M^{2} Z_{\mu}^{\prime} Z^{\mu} \\
& -\frac{e}{c_{W} s_{W}} \frac{g_{Z^{\prime}}}{g_{Z}} \sum_{i} \bar{\psi}_{i L} \gamma^{\mu} C_{L}^{i} \psi_{i L} Z_{\mu}^{\prime}+\bar{\psi}_{i R} \gamma^{\mu} C_{R}^{i} \psi_{i R} Z_{\mu}^{\prime}
\end{aligned}
$$

In this form, $F$ and $F^{\prime}$ are field strength tensors for the $Z^{0}$ and the $Z^{\prime}$, respectively, and $C_{L}$ and $C_{R}$ are the left and right couplings of the $Z^{\prime}$ to the SM fermion fields, $\psi_{L, R}$. The constants $c_{W}$ and $s_{W}$ are the sine and cosine of the weak angle. The term with $\sin \chi$ represents the kinetic mixing that is possible in some models.

The term including $\delta M^{2}$ arises in some models as a mixing between the $Z^{\prime}$ and the SM $Z^{0}$, given by $\delta M^{2}=\epsilon\left(M_{Z^{\prime}}^{2}-M_{Z}^{2}\right)$, for mixing angle $\epsilon{ }^{49}$ This term is constrained by precision measurements of the SM $Z$ properties, as will be discussed in Section 4.1.1. 


\subsection{Model Descriptions}

\subsubsection{String Inspired $E_{6}$ Models}

Though String Theory may not be experimentally verifiable, it has inspired a set of models of BSM. In the $E_{6}$ models, ${ }^{20,21}$ inspired by the heterotic string group, the $E_{6}$ group breaks either directly to an $S O(10) \times U(1)_{\psi}$ or along the path $S O(10) \rightarrow$ $S U(5) \times U(1)_{\chi}$, resulting in the $\psi$ and $\chi$ models, respectively.

However, a more general case can be developed by allowing mixing between these two forms. In the general case, the charge associated with a particle is given by $Q_{E_{6}}\left(\theta_{E_{6}}\right)=\cos \theta_{E_{6}} Q_{\chi}+\sin \theta_{E_{6}} Q_{\psi}$, with the stipulation that $\pi / 2 \leq \theta_{E_{6}} \leq \pi / 2$. An interesting situation occurs when $\theta_{E_{6}}=\arctan (-\sqrt{5 / 3})$. At this value of $\theta_{E_{6}}{ }^{21}$ originating from string theory, the $Z_{\eta}^{\prime}$ decouples from up type quarks.

The charges for the $E_{6}$ models are listed in Table 3.1. In the notation given, the right handed SM fermions have the negative charge of the corresponding left handed conjugate $\left(Q_{u_{R}}=-Q_{\bar{u}_{L}}\right)$, and the couplings $C_{L}$ and $C_{R}$ are the corresponding charge for the left and right fermion.

The coupling strength for the $E_{6}$ models is a free parameter, with the stipulation that $g_{Z^{\prime}} / g_{Z} \leq \sqrt{\frac{5}{3} \mathrm{~s}_{W}^{2}} \cdot 15$ The maximum value, $g_{Z^{\prime}} / g_{Z}=\sqrt{\frac{5}{3} \mathrm{~s}_{W}^{2}}$, is used within this thesis as was done by Cvetič and Godfrey. ${ }^{15}$ 
Table 3.1: Couplings for the $E_{6} \psi$ and $\chi$ models. Couplings to right handed fermions are the negative of the left handed conjugates. These groups allow for several new fermions, including: $D$, a down type quark that transforms as a singlet under $S U(2)_{L},\left(E^{0}, E^{-}\right)$, forming a lepton doublet that transforms as a vector under $S U(2)_{L}$, and $N$ and $S$, massive Weyl neutrinos.

\begin{tabular}{|c|c||c|c|c|}
\hline$S O(10)$ Rep. & $S U(5)$ Rep. & Particle Designation & $2 \sqrt{6} Q_{\psi}$ & $2 \sqrt{10} Q_{\chi}$ \\
\hline \hline 16 & 10 & $\left(u, d, \bar{u}, e^{+}\right)_{L}$ & -1 & 1 \\
& $5^{*}$ & $\left(\bar{d}, \nu, e^{-}\right)_{L}$ & 3 & 1 \\
& 1 & $\bar{N}_{L}$ & -5 & 1 \\
\hline 10 & 5 & $\left(D, \bar{E}^{0}, E^{+}\right)_{L}$ & 2 & -2 \\
& $5^{*}$ & $\left(\bar{D}, E^{0}, E^{-}\right)_{L}$ & -2 & -2 \\
\hline 1 & 1 & $S_{L}^{0}$ & 0 & 4 \\
\hline
\end{tabular}




\subsubsection{Left Right and Alternate Left Right Symmetric Models}

In the Left Right Symmetric Models ${ }^{14,15}$ (LRSM and Alternate/ALRSM), the higher group $S O(10)$ (or even the $E_{6}$ group) breaks first to an intermediate gauge group that includes a right handed $S U(2)$. The resulting QCD and Electroweak groups above the $Z^{\prime}$ level then becomes $S O(10) \rightarrow S U(3)_{c} \times S U(2)_{L} \times S U(2)_{R} \times U(1)_{B-L}$ (where $B-L$ is the baryon minus lepton numbers). The ALRSM arises due to a different assignment of isospin of the fermions under the $S U(2)_{R} \cdot{ }^{16-19}$

The couplings of the fermions under the new gauge group are then given by the following relation, for both the LRSM and the ALRSM:

$$
\begin{aligned}
& C_{L}=\mathrm{s}_{W}^{2}\left(T_{3 L}-Q_{e m}\right) \\
& C_{R}=\kappa^{2}\left(1-\mathrm{s}_{W}^{2}\right) T_{3 R}-s_{W}^{2} Q_{e m}
\end{aligned}
$$

In this form, $0.55 \leq \kappa^{2} \equiv\left(\frac{g_{R}}{g_{L}}\right)^{2} \leq 1(2)$ (value of 1 or 2 depends on whether the symmetry is broken by a Higgs doublet or triplet), and $T_{3 L(R)}$ are the isospin assignments of the fermions under the $S U(2)_{L(R)}$. This thesis shall consider $\kappa=1$, as is common in the literature on the subject. This sets the coupling strength to the left handed $S U(2)$ the same as the right handed $S U(2)$.

In the LRSM, the assignments of $T_{3 L}$ are the same as those in the SM, and the right handed fermions form quark and lepton doublets of $\left(u_{R}, d_{R}\right)$ and $\left(\nu_{R}, e_{R}\right)$ with $T_{3 R}$ isospin assignments of $(1 / 2,-1 / 2)$ for both.

In the ALRSM, the assignments of $T_{3 R}$ differ from the LRSM. The assignment of $T_{3 L}$ is the same as in the SM, but $T_{3 R}$ is assigned as $T_{3 R}(u)=1 / 2, T_{3 R}(d)=0$, $T_{3 R}(e)=-1 / 2$, and $T_{3 R}(\nu)=-1 / 2$.

The coupling strength for both models are identical, and related to the coupling 
for the $Z$ via the relation:

$$
\frac{g_{Z^{\prime}}}{g_{Z}}=\frac{1}{\sqrt{\kappa^{2}-\left(1+\kappa^{2}\right) \mathrm{s}_{W}^{2}}}
$$

\subsubsection{HARV Model}

The HARV model, otherwise known as the Un-unified Standard Model, ${ }^{34,35}$ arises from a separation of the quarks and leptons into distinct groups. The gauge symmetry for the Electroweak sector in this model is $S U(2)_{l} \times S U(2)_{q} \times U(1)_{Y}$. The HARV $Z^{\prime}$ is entirely left handed, and the left handed quarks transform as a doublet under $S U(2)_{q}$, and left handed leptons transform as a doublet under $S U(2)_{l}$. The left handed particles transform as singlets under the opposite named $S U(2)$ symmetry.

The couplings of the fermions in the extra neutral gauge sector are given by:

$$
C_{L, H A R V}=\frac{T_{3 q}}{\tan \phi}-\tan \phi T_{3 l}
$$

The parameter $\phi$ is limited to the range $0.22 \leq \sin \phi \leq 0.99$. A value of $\sin \phi=0.5$ was chosen for analysis within this thesis as it is roughly the midpoint in the allowed range, and as was done in Cvetič and Godfrey. ${ }^{15}$ The coupling strength for the HARV model is given by $\frac{g_{Z^{\prime}}}{g_{Z}}=\mathrm{c}_{W}$.

\subsubsection{Topcolor Models}

Topcolor models ${ }^{24-26}$ arise as a potential explanation for the unusually large top quark mass, by suggesting that the third generation particles are treated differently in the process involved in EWSB. Below some scale, it is assumed that the gauge group is broken from $S U(3)_{1} \times S U(3)_{2} \times U(1)_{Y 1} \times U(1)_{Y 2} \times S U(2)_{L} \rightarrow S U(3)_{c} \times S U(2)_{L} \times$ 
$U(1)_{Y}$. In this schema, the breaking occurs via $S U(3)_{1} \times S U(3)_{2} \rightarrow S U(3)_{c}$ and $U(1)_{Y 1} \times U(1)_{Y 2} \rightarrow U(1)_{Y}$.

In general, any combination of generations can couple to the group structures labeled by subscripts 1 or 2 . The generations can be assigned to $U(1)_{1}$ and $U(1)_{2}$ as either $(1,2)_{1}+(3)_{2}$ or $(2)_{1}+(1,3)_{2}$ or even ()$_{1}+(1,2,3)_{2}$, and still produce a physical result. However, this thesis will concentrate on the $(1,2)_{1}+(3)_{2}$ form described by Harris et al. ${ }^{24}$

The large mass of the top quark, and subsequently smaller mass of the bottom quark, is then generated by a "seesaw" mechanism that results in the preferential formation of a $t \bar{t}$ condensate rather than a $b \bar{b}$ condensate. ${ }^{24,50,51}$ This is accomplished naturally via the inclusion of an extra neutral gauge boson that couples attractively between $t \bar{t}$ and repulsively between $b \bar{b}$. This is a more attractive physical principle, as it requires a minimum of fine tuning.

The couplings of the SM fermions to the new $Z^{\prime}$ are then given in Table 3.2. The coupling strengths of the Topcolor models are given by $\frac{g_{Z^{\prime}}}{g_{Z}}\left(\right.$ gen.1\&2) $=\frac{g_{1}}{g_{Z}}=$ $\frac{1}{2} \mathrm{~s}_{W} \tan \theta_{H}$ and $\frac{g_{Z^{\prime}}}{g_{Z}}\left(\right.$ gen.3) $=\frac{g_{2}}{g_{Z}}=\frac{1}{2} \mathrm{~S}_{W} \cot \theta_{H}$, corresponding to $U(1)_{1}$ and $U(1)_{2}$ respectively. The parameter $\theta_{H}$ is a free parameter that scales the coupling strengths of the two sectors as $g_{1} / g_{2}=\tan ^{2} \theta_{H}$ and producing a preferential coupling to the third generation for low values of $\tan \theta_{H}$. For the purpose of this thesis, a value of $\tan \theta_{H}=0.577$, such that $\cot \theta_{H}^{2}=3.00$. This is roughly the midpoint between the values chosen by Harris et $a l^{24}$ for $M_{Z^{\prime}}=800 \mathrm{GeV}$ and $M_{Z^{\prime}}=\infty \mathrm{GeV}$. This is fairly arbitrary and was chosen to approximately maximize the $Z^{\prime}$ peak height. A more complete analysis would consider a variety of possible values. 
Table 3.2: Couplings of the left and 'right handed SM fermions to the extra neutral gauge sector in the Topcolor model with a $(1,2)_{1}+(3)_{2}$ generation coupling gauge sector.

\begin{tabular}{|c|c|c|}
\hline Fermion & $C_{L, T C}$ & $C_{R, T C}$ \\
\hline \hline$u, c$ & $+\frac{1}{3}$ & $+\frac{4}{3}$ \\
\hline$d, s$ & $+\frac{1}{3}$ & $-\frac{2}{3}$ \\
\hline$t$ & $-\frac{1}{3}$ & $-\frac{4}{3}$ \\
\hline$b$ & $-\frac{1}{3}$ & $+\frac{2}{3}$ \\
\hline$e, \mu$ & -1 & -2 \\
\hline$\nu_{e}, \nu_{\mu}$ & -1 & 0 \\
\hline$\tau$ & +1 & +2 \\
\hline$\nu_{\tau}$ & +1 & 0 \\
\hline
\end{tabular}

\subsubsection{Littlest Higgs Model}

The Little Higgs Models ${ }^{27-33}$ are designed as possible solutions to the hierarchy problem by introducing the Higgs boson as a pseudo-Nambu-Goldstone boson that is broken at the TeV level to the SM Higgs doublet. A number of new particles are posited in these models at masses above the breaking scale, including new scalar particles, new gauge bosons, and another heavy quark similar to the top. The Littlest Higgs Model (LHM) is a variant of the Little Higgs Models in which the SM $S U(2)_{L} \times U(1)_{Y}$ is formed from the breaking of a gauged $(S U(2) \times U(1)) \times(S U(2) \times U(1))$ product group that is embedded within an $S U(5)$ global symmetry.

The $Z^{\prime}$ arising from this model is typically denoted as $Z_{H}$ in the literature, 
as will be done in this thesis when singling out this model in discussion. The $Z_{H}$ couples purely to left handed particles, with couplings given by $C_{L, L H M}=T_{3}$, the third component of isospin, and $C_{R, L H M}=0$.

The coupling strength for the LHM is given by the following relation:

$$
\frac{g_{Z_{H}}}{g_{Z}}=\mathrm{c}_{W} \cot \theta_{H}
$$

The parameter $\theta_{H}$ is defined as the mixing angle between the two $S U(2)$ groups of the model, and is determined by the relationship $\tan \theta_{H}=g_{2} / g_{1}$. In general, this is a free parameter; the value $\cot \theta_{H}=1$ will be used (yielding $\theta_{H}=45^{\circ}$ ) as this minimizes the branching fraction to $Z H$ and $W W$. Electroweak precision data suggests a relatively small value of $\cot \theta_{H} \cdot{ }^{27}$ In general, this smaller value would reduce the expected production at the LHC.

\subsubsection{Simplest Little Higgs Model}

The Simplest Little Higgs Model (SLHM) ${ }^{27,52-54}$ arises from a different gauge structure possible in Little Higgs Models than the one used in the LHM. This model is listed in Han et $a l^{27}$ as the Simple Group with generation universal couplings. Instead of the breaking of a product group to the SM Electroweak symmetry, the SLHM purports that the breaking scheme follows $S U(3)_{W} \times U(1)_{\chi} \rightarrow S U(2)_{L} \times U(1)_{Y} . \mathrm{M}$. Schmalt ${ }^{54}$ suggests that the resulting $S U(3)$ gauge bosons would then cancel out quadratic Higgs mass divergences that arise from loops involving $W$ and $Z$ bosons from the SM.

This model predicts the existence of five gauge bosons at large masses, including a neutral extra gauge boson. The couplings of the SM particles to the extra neutral sector are listed in Table 3.3. The coupling strength is then given by the 
Table 3.3: Couplings of the left and right handed chiral fermions in the $S U(3) \times U(1)$ of the Simplest Little Higgs model.

\begin{tabular}{|c|c|c|}
\hline Fermion & $C_{L, S L H M}$ & $C_{R, S L H M}$ \\
\hline \hline$u, c, t$ & $\frac{1}{2}-\frac{1}{3} \mathrm{~s}_{W}^{2}$ & $\frac{2}{3} \mathrm{~s}_{W}^{2}$ \\
\hline$d, s, b$ & $\frac{1}{2}-\frac{1}{3} \mathrm{~s}_{W}^{2}$ & $-\frac{1}{3} \mathrm{~s}_{W}^{2}$ \\
\hline$e, \mu, \tau$ & $\frac{1}{2}-\mathrm{s}_{W}^{2}$ & $-\mathrm{s}_{W}^{2}$ \\
\hline$\nu_{e}, \nu_{\mu}, \nu_{\tau}$ & $\frac{1}{2}-\mathrm{s}_{W}^{2}$ & 0 \\
\hline
\end{tabular}

relation:

$$
\frac{g_{Z^{\prime}}}{g_{Z}}=\frac{1}{\sqrt{3-4 \mathrm{~s}_{W}}}
$$

Unlike many other models, the coupling strength for this model is well defined, rather than a free parameter.

\subsubsection{Anomaly Free Simple Little Higgs Model}

The Anomaly Free Simple Little Higgs Model (ASLHM $)^{27,55}$ arises similarly to the SLHM, but allows for distinct couplings for the third generation quarks than for the first two generations. Lepton couplings remain identical throughout the generations. The coupling strength remains the same as in the SLHM, with the couplings listed in Table 3.4 . 
Table 3.4: Couplings of the left and right handed chiral fermions in the $S U(3) \times U(1)$ of the Simple Little Higgs model with non-generation universal couplings.

\begin{tabular}{|c|c|c|}
\hline Fermion & $C_{L, A S L H M}$ & $C_{R, A S L H M}$ \\
\hline \hline$t$ & $\frac{1}{2}-\frac{1}{3} \mathrm{~s}_{W}^{2}$ & $\frac{2}{3} \mathrm{~s}_{W}^{2}$ \\
\hline$b$ & $\frac{1}{2}-\frac{1}{3} \mathrm{~s}_{W}^{2}$ & $-\frac{1}{3} \mathrm{~s}_{W}^{2}$ \\
\hline$u, c$ & $-\frac{1}{2}+\frac{2}{3} \mathrm{~s}_{W}^{2}$ & $\frac{2}{3} \mathrm{~s}_{W}^{2}$ \\
\hline$d, s$ & $-\frac{1}{2}+\frac{2}{3} \mathrm{~s}_{W}^{2}$ & $-\frac{1}{3} \mathrm{~s}_{W}^{2}$ \\
\hline$e, \mu, \tau$ & $\frac{1}{2}-\mathrm{s}_{W}^{2}$ & $-\mathrm{s}_{W}^{2}$ \\
\hline$\nu_{e}, \nu_{\mu}, \nu_{\tau}$ & $\frac{1}{2}-\mathrm{s}_{W}^{2}$ & 0 \\
\hline
\end{tabular}

\subsubsection{3-3-1 Models}

The name " $3-3-1$ " 22,23 is given to the set of models that arise from the breaking of the larger structure of $S U(3)_{c} \times S U(3) \times U(1)_{X}$ to the SM QCD and EW groups. There are several ways that this structure can manifest itself, though this thesis will deal with two versions - one in which there are two extra up type quarks and a single extra down type quark, and one in which there are two extra down type quarks and a single extra up type quark.

The 3-3-1 models have some interesting consequences, including the prediction of new physics close to the electroweak symmetry breaking scale, a relationship between the number of families with the number of colors, and potentially a reason for a limit on $\sin \theta_{W}$.

The couplings of the SM fermions are listed in Table 3.5 for each of the two 
Table 3.5: The labels (1) and (2) refer to the two versions examined, (1) indicating the model with 2 extra up and 1 extra down type quark, and (2) indicated the model with 1 extra up and 2 extra down.

\begin{tabular}{|c|c|c|c|c|}
\hline Fermion & $C_{L, 331}(1)$ & $C_{R, 331}(1)$ & $C_{L, 331}(2)$ & $C_{R, 331}(2)$ \\
\hline \hline$c, t$ & $\frac{1}{2}-\frac{1}{3} \mathrm{~s}_{W}^{2}$ & $\frac{2}{3} \mathrm{~s}_{W}^{2}$ & $\frac{1}{2}-\frac{1}{3} \mathrm{~s}_{W}^{2}$ & $\frac{2}{3} \mathrm{~s}_{W}^{2}$ \\
\hline$s, b$ & $\frac{1}{2}-\frac{1}{3} \mathrm{~s}_{W}^{2}$ & $-\frac{1}{3} \mathrm{~s}_{W}^{2}$ & $\frac{1}{2}-\frac{1}{3} \mathrm{~s}_{W}^{2}$ & $-\frac{1}{3} \mathrm{~s}_{W}^{2}$ \\
\hline$u$ & $-\frac{1}{2}+\frac{2}{3} \mathrm{~s}_{W}^{2}$ & $\frac{2}{3} \mathrm{~s}_{W}^{2}$ & $\frac{1}{2}-\frac{1}{3} \mathrm{~s}_{W}^{2}$ & $\frac{2}{3} \mathrm{~s}_{W}^{2}$ \\
\hline$d$ & $-\frac{1}{2}+\frac{2}{3} \mathrm{~s}_{W}^{2}$ & $-\frac{1}{3} \mathrm{~s}_{W}^{2}$ & $\frac{1}{2}-\frac{1}{3} \mathrm{~s}_{W}^{2}$ & $-\frac{1}{3} \mathrm{~s}_{W}^{2}$ \\
\hline$e, \mu, \tau$ & $-\frac{1}{2}$ & $-\mathrm{s}_{W}^{2}$ & $\frac{1}{2}-\mathrm{s}_{W}^{2}$ & $-\mathrm{s}_{W}^{2}$ \\
\hline$\nu_{e}, \nu_{\mu}, \nu_{r}$ & $\frac{1}{2}$ & $-\mathrm{s}_{W}^{2}$ & $\frac{1}{2}-\mathrm{s}_{W}^{2}$ & $-\mathrm{s}_{W}^{2}$ \\
\hline
\end{tabular}

models listed, with coupling strength of the model given by:

$$
\frac{g_{Z^{\prime}}}{g_{Z}}=\frac{1}{\sqrt{3-4 \mathrm{~s}_{W}^{2}}}
$$

From this, we can see that we require the relation $s_{W}^{2}<3 / 4$. Some $3-3-1$ models include the stipulation that $\mathrm{s}_{W}^{2}<1 / 4$ - surprisingly, the experimentally determined value of $s_{W}^{2}$ is quite close to this upper bound.

\subsection{9 $S U(2)_{h} \times S U(2)_{l}$ Models (HLSUM)}

The extended weak group could take the general form of $S U(2)_{h} \times S U(2)_{l}$, which would correspond to such groups as non-commuting Technicolor ${ }^{40-42}$ and Topflavour models. ${ }^{43-45}$ Within these models, the first two generations transform as doublets under the $S U(2)_{l}$, denoted the "light" $S U(2)$, while the third generation transforms as a doublet under the $S U(2)_{h}$, denoted the "heavy" $S U(2)$, and each generation 
transforms as singlet under the opposite $S U(2)$. From this, the breaking scheme follows $S U(2)_{h} \times S U(2)_{l} \rightarrow S U(2)_{L} \times U(1)_{Y}$ below some scale $u$ to produce the SM EW interactions.

The electric charge operator for the $U(1)_{\text {em }}$ group is then given by $Q=T_{3 h}+$ $T_{3 l}+Y$. Defining the mixing angle between the $S U(2)$ groups as $\phi$, the $Z^{\prime}$ couplings are then given by:

$$
C_{L, H L S U M}=\left(-\tan \phi T_{3 h}+\cot \phi T_{3 l}\right)
$$

The coupling strength for this model is the SM coupling $g$. This results in preferential coupling to third generation fermions for large values of $\sin \phi$ ( $\sin \phi=0.9$ used for strong preferential third generation coupling*).

\subsubsection{0 $U(1)_{h} \times U(1)_{l}$ Models (HLUM)}

Models that include an extra $U(1)$ gauge group can also result in a $Z^{\prime}$ with nongeneration universal couplings, with a distinct third generation (h) and first/second generation (1) coupling as with the previous model. Specifically, models of Topcolor assisted Technicolor ${ }^{26,36-38}$ and flavour-universal Topcolor assisted Technicolor ${ }^{38,39}$ can be generated in this form. Symmetry breaking occurs via $S U(2)_{W} \times U(1)_{h} \times$ $U(1)_{l} \rightarrow S U(2)_{L} \times U(1)_{Y}$, with third generation particles coupling to the $U(1)_{h}$ and with the first two generations coupling to the $U(1)_{l}$.

Couplings for the SM fermions within the extra neutral gauge sector are defined

*This is to examine the situation of distinct preference for third generation final states. A more moderate situation may be more realistic, and could be examined in a more complete analysis of this specific group structure. 
as:

$$
\begin{aligned}
& C_{L, H L U M}=\left(-\cot \chi Y_{h}+\tan \chi Y_{l}\right) \\
& C_{R, H L U M}=0
\end{aligned}
$$

The angle, $\chi$, represents the mixing between the two hypercharge sectors such that $\cot \chi=\left(\frac{g_{h}}{g_{l}}\right)^{2}$. Preferential coupling to third generation fermions then occurs for large values of $\cos \chi\left(\cos \chi=0.9\right.$ used for strong third generation coupling $\left.{ }^{\dagger}\right)$. The hypercharge assignments for $Y_{h, l}$ are given by the standard hypercharge values as for the $Z$, and the coupling strength is then given as the $\mathrm{SM} g^{\prime}$ (i.e.: $\frac{g_{Z^{\prime}}}{g Z}=\frac{g^{\prime}}{g Z}$ ).

\subsubsection{Benchmark: Sequential Standard Model}

The Sequential Standard Model is not intended to be considered literally; this model assumes all $Z^{\prime}$ coupling strength and charges are identical to the SM $Z$, but with a larger gauge boson mass.

This results in the couplings listed in Table 3.6 and in the following relationship for the coupling strength:

$$
\frac{g_{Z^{\prime}}}{g_{Z}}=1
$$

\footnotetext{
${ }^{\dagger}$ As with the previous model, this is chosen specifically to examine the scenario of distinct distinct preference for third generation final states.
} 
Table 3.6: The $Z^{\prime}$ in the Sequential Standard Model is identical to that of the Standard Model $Z$ except with a larger mass.

\begin{tabular}{|c|c|c|}
\hline Fermion & $C_{L, S S M}$ & $C_{R, S S M}$ \\
\hline \hline$u, c, t$ & $\frac{1}{2}-\frac{2}{3} \mathrm{~s}_{W}^{2}$ & $-\frac{2}{3} \mathrm{~s}_{W}^{2}$ \\
\hline$d, s, b$ & $-\frac{1}{2}+\frac{1}{3} \mathrm{~s}_{W}^{2}$ & $\frac{1}{3} \mathrm{~s}_{W}^{2}$ \\
\hline$e, \mu, \tau$ & $-\frac{1}{2}+\mathrm{s}_{W}^{2}$ & $\mathrm{~s}_{W}^{2}$ \\
\hline$\nu_{e}, \nu_{\mu}, \nu_{\tau}$ & $\frac{1}{2}$ & 0 \\
\hline
\end{tabular}




\section{Chapter 4}

\section{$Z^{\prime}$ Observables}

This chapter describes the formulae for a number of observables and discusses kinematic cuts and analysis techniques for determining the nature of the $Z^{\prime}$. The first section will include a review of observables and techniques from the literature, while the sections that follow will be specific to my own research. All calculations of DrellYan processes involved were done at tree level and incorporate correction factors for higher order loop diagrams in the running of coupling strengths. Drell-Yan processes are the s-channel production of leptonic final states from hadronic collisions through either a $Z$ or $\gamma$, but can be extended to include a third neutral boson (i.e.: $Z^{\prime}$ ).

I would like to clarify the use of the following notation that will be used in the following sections for couplings. In general, I use the left and right fermion coupling strengths, normalized to the $Z$ coupling strength. These relate to the $Z^{\prime}$ via the following:

$$
g_{L(R)}^{f}=\frac{g_{Z^{\prime}}}{g_{Z^{0}}} C_{L(R)}^{f}
$$

where $C_{L(R)}^{f}$ is the coupling of the left (right) chiral fermion, of flavour $f$, to the $Z^{\prime}$ in a specific model. 
Additionally, I purport that observables in the form of ratios of processes provide the most distinct method for distinguishing between models since they cancel out many theoretical and experimental uncertainties that complicate the analysis. The calculations of observables in Chapter 5 do not take into account experimental concerns (such as the effect of detector resolution), and a corresponding full experimental simulation may result in a different estimation of uncertainties.

Several terms are used in the following sections that may require definition.

Rapidity, $y$ - a unitless measure of the boost of the interaction, as an unbalanced

interaction between interacting particles. $y \equiv \frac{1}{2} \ln \frac{E+p_{\|}}{E-p_{\|}}$

Transverse Energy/Momentum, $E_{T}, p_{T}$ - the component of energy/momentum of the final state particles that is perpendicular to the incoming particle beams.

Parton - A particle (fermion or boson) component of a hadron that carries some momentum of the hadron. In the case of a proton, this is primarily the $u$ and $d$ quarks that comprise the general structure, and the gluons that maintain the bound state, but also includes 'sea' quark contributions of $s, c$, and $b$ quarks (sea $t$ quark contributions are neglected in this thesis).

\subsection{Existing Observables}

Since the LHC is a hadron collider, a large QCD background is expected. The QCD interaction strength is approximately an order of magnitude larger than the Electroweak interaction strength, and so the production of quark jets at the LHC is expected to be primarily from strong interactions. Alternatively, dilepton production at the LHC will occur primarily via Drell-Yan processes - the annihilation of quarkantiquark pairs via an EW vertex and the production of lepton final states. Since 
this involves two EW vertices, as opposed to two QCD vertices, the EW backgrounds should be around two orders of magnitude smaller.

The cleanest channel for detection of an extra neutral gauge boson at the LHC is via an excess of lepton pair production. The expected interaction of a $Z^{\prime}$ would result in a resonance at the mass of the $Z^{\prime}$ and a very distinctive Breit-Wigner peak in the number of events occurring near that energy scale.

For most models, dilepton production is the best channel for discovery of a $Z^{\prime}$. However, there is a small subset of models that are leptophobic - the $Z^{\prime}$ does not couple, or couples very weakly to leptons. For these models, the leptonic channel will not be sufficient. Detection of such a gauge boson would then require an analysis of quark final states. This will be discussed in further detail in Section 4.2.

\subsubsection{Decay Width and Branching Ratios}

To a first approximation, the decay width of a general $Z^{\prime}$ can be calculated at tree level. In general, there may be more fermions than those included in the SM, and while it is possible that these fermions are kinematically accessible by a $Z^{\prime}\left(M_{Z^{\prime}}>2 m_{f^{\prime}}\right)$, decay widths for the $Z^{\prime}$ models discussed will be calculated from decays only to SM fermions. Additionally, decays to the SM EW gauge bosons are possible through mixing with the $Z$, and, in general, should contribute to the decay width. However, high precision measurements of the properties of the $Z,{ }^{56}$ at SLC and LEP I, have constrained the mixing angle to:

$$
\epsilon=\frac{\delta M_{Z Z^{\prime}}^{2}}{M_{Z^{\prime}}^{2}-M_{Z}^{2}} \leq 10^{-3}
$$

As a result, this can be neglected in the decay width for many models to the precision of the analysis of this thesis. Within the Little Higgs models considered, this effect is 
more complicated. The longitudinal final state gauge bosons (i.e.: $W_{L}^{+} W_{L}^{-}$and $Z_{L} H$ ) have an enhancement factor that partially cancels out the $\frac{\delta M_{Z Z^{\prime}}^{2}}{M_{Z^{\prime}}^{2}}$ suppression. In the LHM, this is $0 \%$ for $\theta_{H}=45^{\circ}$, but for the SLHM and ASLHM it is $0.87 \%$ and $1.1 \%$, respectively. ${ }^{27}$ This was not taken into account in the calculation of widths for this thesis.

The partial widths are defined in Equation (4.3), with the total width calculated as the sum of partial widths.

$$
\begin{aligned}
\Gamma_{f}\left(Z^{\prime} \rightarrow f \bar{f}\right) & =\frac{\beta_{f}}{8 \pi M_{Z^{\prime}}}|\mathcal{M}|^{2} \\
& =\frac{N_{c} \alpha_{e m} M_{Z^{\prime}} \beta_{f}}{6 c_{W}^{2} s_{W}^{2}}\left(\left(g_{R}^{f 2}+g_{L}^{f 2}\right)\left(1-\frac{m_{f}^{2}}{M_{Z^{\prime}}^{2}}\right)+6 g_{L}^{f} g_{R}^{f} \frac{m_{f}^{2}}{M_{Z^{\prime}}^{2}}\right) \\
\Gamma_{Z^{\prime}} & =\sum_{f} \Gamma_{f}
\end{aligned}
$$

In this form, $\beta_{f}=\sqrt{1-4 m_{f}^{2} / M_{Z^{\prime}}^{2}}$, and $N_{c}$ is the color factor for quarks.

In general, we expect that $m_{f}^{2} / M_{Z^{\prime}}^{2} \ll 1$ for most fermions, resulting in $\beta_{f} \approx 1$. However, for a low mass $Z^{\prime}$, the top quark mass may be within an order of magnitude of the mass of the extra gauge boson. Table 4.1 gives the ratio of $\Gamma_{Z^{\prime}} / M_{Z^{\prime}}$ for each model and for $M_{Z^{\prime}}=1000,3000 \mathrm{GeV}$ to illustrate the effect of the mass term. In general, this is a minor effect. Primarily, this factor reduces the partial width to top quarks at low $M_{Z^{\prime}}^{2}$, which only has a largely noticeable effect in models with preferential couplings to third generation fermions.

From this, we can define the branching ratio as the probability of decay into a specific final state, given by the ratio of the partial width divided by the total width:

$$
B R\left(Z^{\prime} \rightarrow f \bar{f}\right)=\frac{\Gamma_{f}}{\Gamma_{Z^{\prime}}}
$$

The production cross section of a $Z^{\prime}$ will have interference effects from $\gamma$ and $Z$. However, if we consider only those events with $M_{f \bar{f}} \approx M_{Z^{\prime}}$, the total cross section 
Table 4.1: Ratio of $\Gamma_{Z^{\prime}} / M_{Z^{\prime}}$ for each considered model at $M_{Z^{\prime}}=1$ and $3 \mathrm{TeV}$. The table illustrates the decreasing influence of kinematic terms in the width.

\begin{tabular}{|l|c|c|}
\cline { 2 - 3 } \multicolumn{1}{c|}{} & $\Gamma_{Z^{\prime}} / M_{Z^{\prime}} @ 1000 \mathrm{GeV}$ & $\Gamma_{Z^{\prime}} / M_{Z^{\prime}} @ 3000 \mathrm{GeV}$ \\
\hline \hline Model & $\left(\times 10^{-3}\right)$ & $\left(\times 10^{-3}\right)$ \\
\hline E6 Model $\chi$ & 11.8 & 11.8 \\
\hline E6 Model - $\psi$ & 6.30 & 6.48 \\
\hline LR Symmetric & 5.28 & 5.39 \\
\hline Alt. LR Symmetric & 20.7 & 20.9 \\
\hline HARV Model & 14.4 & 14.6 \\
\hline Sequential SM & 79.8 & 80.7 \\
\hline Topcolor $(1,2)+(3)$ & 30.0 & 30.4 \\
\hline Littlest Higgs Model & 60.2 & 60.3 \\
\hline Simplest Little Higgs & 34.1 & 34.4 \\
\hline Anomaly Free Simple LH & 14.7 & 14.7 \\
\hline 3-3-1 $(2 U$ 1D $)$ & 12.7 & 12.7 \\
\hline 3-3-1 $(1 \mathrm{U} 2 \mathrm{D})$ & 14.2 & 14.4 \\
\hline$S U(2)_{h} \times S U(2)_{l}$ Models & 14.7 & 14.7 \\
\hline$U(1)_{h} \times U(1)_{l}$ Models & 52.7 & \\
\hline
\end{tabular}


can be simplified to: $\sigma_{Z^{\prime}}\left(q \bar{q} \rightarrow Z^{\prime} \rightarrow f \bar{f}\right) \longrightarrow \sigma\left(q \bar{q} \rightarrow Z^{\prime}\right) \times B R\left(Z^{\prime} \rightarrow f \bar{f}\right)$. This can be added to a general SM background from $Z$ and $\gamma$.

I have included a QCD and electromagnetic correction factor for the production of fermions from an EW vertex. These correction factors take into account higher order diagrams up to order $\alpha_{s}^{3}$ and $\alpha_{e m}$. The correction factors are given by:

$$
\begin{aligned}
K_{Q C D}^{q} & =1+\frac{\alpha_{s}}{\pi}+1.40923 \frac{\alpha_{s}^{2}}{\pi^{2}}-12.767 \frac{\alpha_{s}^{3}}{\pi^{3}} \\
K_{E W}^{f} & =1+\frac{3 \alpha_{e m}}{4 \pi} Q_{e m}^{f 2}
\end{aligned}
$$

Where $\alpha_{s}$ is the strong coupling constant, calculated at the interaction energy via the $\overline{\mathrm{MS}}$ renormalization scheme in the Particle Data Book. ${ }^{7}$

\subsubsection{Differential Cross Section}

In this thesis, I will explore the production of extra neutral gauge bosons via an s-channel process involving the annihilation of quark-antiquark pairs to produce the $Z^{\prime}$ (Drell-Yan dilepton production, ${ }^{57}$ and EW hadronic final states). The primary process is shown in Figure 4.1.

The parton-level differential cross section for the process $\left(q \bar{q} \rightarrow \gamma, Z, Z^{\prime} \rightarrow f \bar{f}\right)$ is given by: ${ }^{58}$

$$
\frac{d \hat{\sigma}}{d \cos \hat{\theta}}=\frac{\pi \alpha_{e m}^{2} \beta_{f}}{8 c_{W} s_{W} \hat{s}}\left\{\left(1+\beta_{f}^{2} \cos ^{2} \hat{\theta}\right) S_{q}+2 \beta_{f} \cos \hat{\theta} A_{q}+S_{q}^{\prime}\right\}
$$




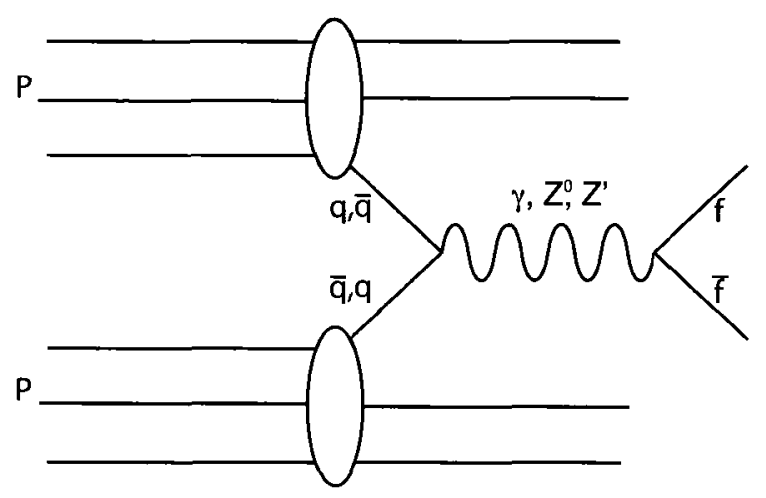

Figure 4.1: Feynman diagram representing the Drell-Yan interaction of $q \bar{q}$ pairs from $p p$ beams to produce final state fermion pairs, $f$, at the LHC.

Where,

$$
\begin{array}{r}
S_{q}, A_{q}=\sum_{i, j=\gamma, Z, Z^{\prime}}\left(\frac{\hat{s}}{\hat{s}-M_{i}^{2}-i \Gamma_{i} M_{i}}\right)\left(\frac{\hat{s}}{\hat{s}-M_{j}^{2}+i \Gamma_{j} M_{j}}\right) \times \\
\left(g_{R i}^{f} g_{R j}^{f} \pm g_{L i}^{f} g_{L j}^{f}\right)\left(g_{R i}^{q} g_{R j}^{q} \pm g_{L i}^{q} g_{L j}^{q}\right) \\
S_{q}^{\prime}=\sum_{i, j=\gamma, Z, Z^{\prime}}\left(\frac{4 m_{f}^{2}}{\hat{s}}\right)\left(\frac{\hat{s}}{\hat{s}-M_{i}^{2}-i \Gamma_{i} M_{i}}\right)\left(\frac{\hat{s}}{\hat{s}-M_{j}^{2}+i \Gamma_{j} M_{j}}\right) \times \\
\left(g_{R i}^{f} g_{L j}^{f}+g_{L i}^{f} g_{R j}^{f}\right)\left(g_{R i}^{q} g_{R j}^{q}+g_{L i}^{q} g_{L j}^{q}\right)
\end{array}
$$

In this form, $S_{q}$ (the summed couplings) and $S_{q}^{\prime}$ provide information on the symmetric production, where $S_{q}^{\prime}$ is a mass dependent term that is non-negligible when the final state fermion mass cannot be neglected (in general $S_{q}^{\prime} \approx 0$, unless the final state considered is a top quark and the mass of the $Z^{\prime}$ is relatively low), and $A_{q}$ (the difference of couplings) is the antisymmetric term.

In general, the energy scale of the interaction, $\hat{s}$, is determined by the interacting partons rather than by the protons, with $s=14 \mathrm{TeV}$. An individual parton, $q$, can contain any fraction, $0<x<1$, of the total momentum of the incoming proton, with varying probability of occurrence. The distribution for the probability of finding a parton with momentum fraction $x$ in a proton at energy scale $Q^{2}$ is 
given by $f_{q(\bar{q})}\left(x, Q^{2}\right)$ for parton $q(\bar{q})$. The annihilation of a quark-antiquark pair in a proton-proton collision at the LHC is then given by:

$$
\frac{d \sigma}{d x_{a} d x_{b} d \cos \hat{\theta}}=\sum_{q} \frac{d \hat{\sigma}_{q}}{d \cos \hat{\theta}} f_{q_{a}}\left(x_{a}, Q^{2}\right) f_{\bar{q}_{b}}\left(x_{b}, Q^{2}\right)+(a \leftrightarrow b, \hat{\theta} \rightarrow \pi-\hat{\theta})
$$

However, typically this parameterization of the differential cross section does not adequately represent measurable properties at the LHC. A more representative form describes the differential cross section in terms energy scale/invariant mass $M$ $\left(\hat{s}=Q^{2}=M^{2}\right)$, rapidity $y\left(y=\ln \left(\sqrt{\frac{x_{a}}{x_{b}}}\right)\right)$, and angular dependence $\theta$. In this form, the momentum fractions are given by the relationship $x_{a, b}=\frac{M}{\sqrt{s}} e^{ \pm y}$.

The differential cross section in terms of these variables is then given by:

$$
\begin{gathered}
\frac{d \sigma}{d M d y d \cos \hat{\theta}}=\left(\frac{\pi \alpha_{e m}^{2} \beta_{f}}{12 M^{3}}\right) \times \sum_{q}( \\
\left.\left(1+\beta_{f}^{2} \cos ^{2} \hat{\theta}\right) S_{q}+S_{q}^{\prime}\right) G_{q}^{+}\left(x_{a}, x_{b}, M\right) \\
+2 \beta_{f} \cos \hat{\theta} A_{q} G_{q}^{-}\left(x_{a}, x_{b}, M\right)
\end{gathered}
$$

Where,

$$
G_{q}^{ \pm}\left(x_{a}, x_{b}, M\right)=x_{a} x_{b}\left(f_{q_{a}}\left(x_{a}, M\right) f_{\bar{q}_{b}}\left(x_{b}, M\right) \pm f_{\bar{q}_{a}}\left(x_{a}, M\right) f_{q_{b}}\left(x_{b}, M\right)\right)
$$

The angular dependence can be integrated out of the differential cross section, leaving the positive and negative differential cross section:

$$
\frac{d \sigma^{ \pm}}{d \overline{M d y}}=\left[\int_{0}^{1} \pm \int_{-1}^{0}\right] d \cos \hat{\theta} \frac{d \sigma}{d M d y d \cos \hat{\theta}}
$$

This final form and notation will be used throughout the rest of this thesis, as the positive and negative differential segregates the symmetric $\left(\sigma^{+} \propto S_{q}, S_{q}^{\prime}\right)$ from antisymmetric $\left(\sigma^{-} \propto A_{q}\right)$ terms. Since these terms depend differently on the couplings between the $Z^{\prime}$ and the fermions, they are useful to consider separately. 
The invariant mass distribution, found by integrating over rapidity, shows the expected Breit-Wigner peak, while the rapidity distribution, found by integrating over invariant mass, shows the distribution of particles in the detector based on the measurable rapidity. If a $Z^{\prime}$ exists, the signal will be very distinct as a large increase in dilepton events with an invariant mass around the mass of the $Z^{\prime}$. By examining the distribution of these events, a good approximation of the mass and of the decay width of the $Z^{\prime}$ can be obtained.

However, at the LHC, the incoherent sum over interacting quark flavours in the protons means that our theoretical understanding of any observed signal will depend on the left and right couplings for the valence quarks in the proton $(u, d)$, the sea quarks ( $u, d, s, c, b$ primarily), and the final state fermions. This means that there are up to twelve unknown parameters to fit to the observed data. The unique nature of the $Z^{\prime}$ will remain ambiguous with a simple examination of the cross section. It remains the challenge of phenomenologists to determine other methods to distinguish between the various possible models.

\subsubsection{Forward-Backward Asymmetry}

The Forward Backward Asymmetry, $A_{F B}$, is a classic observable covered in most of the literature on the subject. The $A_{F B}$ looks at the difference between the number of events boosted in the direction of the interacting quark to those boosted by the interacting anti-quark.

The theoretical expectation for the $A_{F B}$ is given by:

$$
A_{F B} \equiv \frac{\sigma_{F}-\sigma_{B}}{\sigma_{F}+\sigma_{B}}=\frac{\left(\int_{0}^{y_{\max }} d y \frac{d \sigma^{-}}{d y}-\int_{-y_{\max }}^{0} d y \frac{d \sigma^{-}}{d y}\right)}{\left(\int_{0}^{y_{\max }} d y \frac{d \sigma^{+}}{d y}+\int_{-y_{\max }}^{0} d y \frac{d \sigma^{+}}{d y}\right)}
$$


The value of $A_{F B}$ still depends on the values of the twelve parameters mentioned, however it depends on the difference in the squares of the left and right handed couplings. This provides a second, complementary observable with which to try to uniquely identify the model of the $Z^{\prime}$.

\subsubsection{Ratios - Rapidity Ratio, Rare Decays and Associated Production}

For completeness, I will describe other observables discussed in the literature.

\section{Rapidity Ratio}

A rapidity ratio has been suggested by del Aguila, Cvetič and Langacker ${ }^{3}$ that examines the number of events that occur in two regions of rapidity - the central and outer regions - where the number of events are approximately equal. For a $1 \mathrm{TeV} Z^{\prime}$, they suggest looking at the ratio of events where $|y|<1$ and $|y|>1$, and find:

$$
R_{y} \equiv \frac{\int_{-y}^{y} d y \frac{d \sigma}{d y}}{\int_{y}^{y_{\max }}+\int_{-y_{\max }}^{-y} d y \frac{d \sigma}{d y}} \approx 1.55 \frac{0.64\left(g_{L}^{u 2}+g_{R}^{u 2}\right)+0.36\left(g_{L}^{d 2}+g_{R}^{d 2}\right)}{0.73\left(g_{L}^{u 2}+g_{R}^{u 2}\right)+0.27\left(g_{L}^{d 2}+g_{R}^{d 2}\right)}
$$

This analysis is sensitive to the weighting $\left(g_{L}^{2}+g_{R}^{2}\right)$ of the $\mathrm{u}$ - versus d- quark contributions, and finds its origins in the differences in the rapidity distributions for the interacting quarks. Figure 4.2 shows the distributions of $G_{q}^{ \pm}(y, M)$ vs rapidity at $M=1 \mathrm{TeV}$, for the contributing partons, to illustrate the differences in distributions. The up quark tends to be a wider distribution, while the down quark tends to be more narrow, allowing for this analysis to be effective.

\section{Rare Decays}

Rizzo $^{4}$ suggested considering the rare case of one of the final state particles emitting a SM gauge boson. From comparing these events to the dimuon events, he suggests 


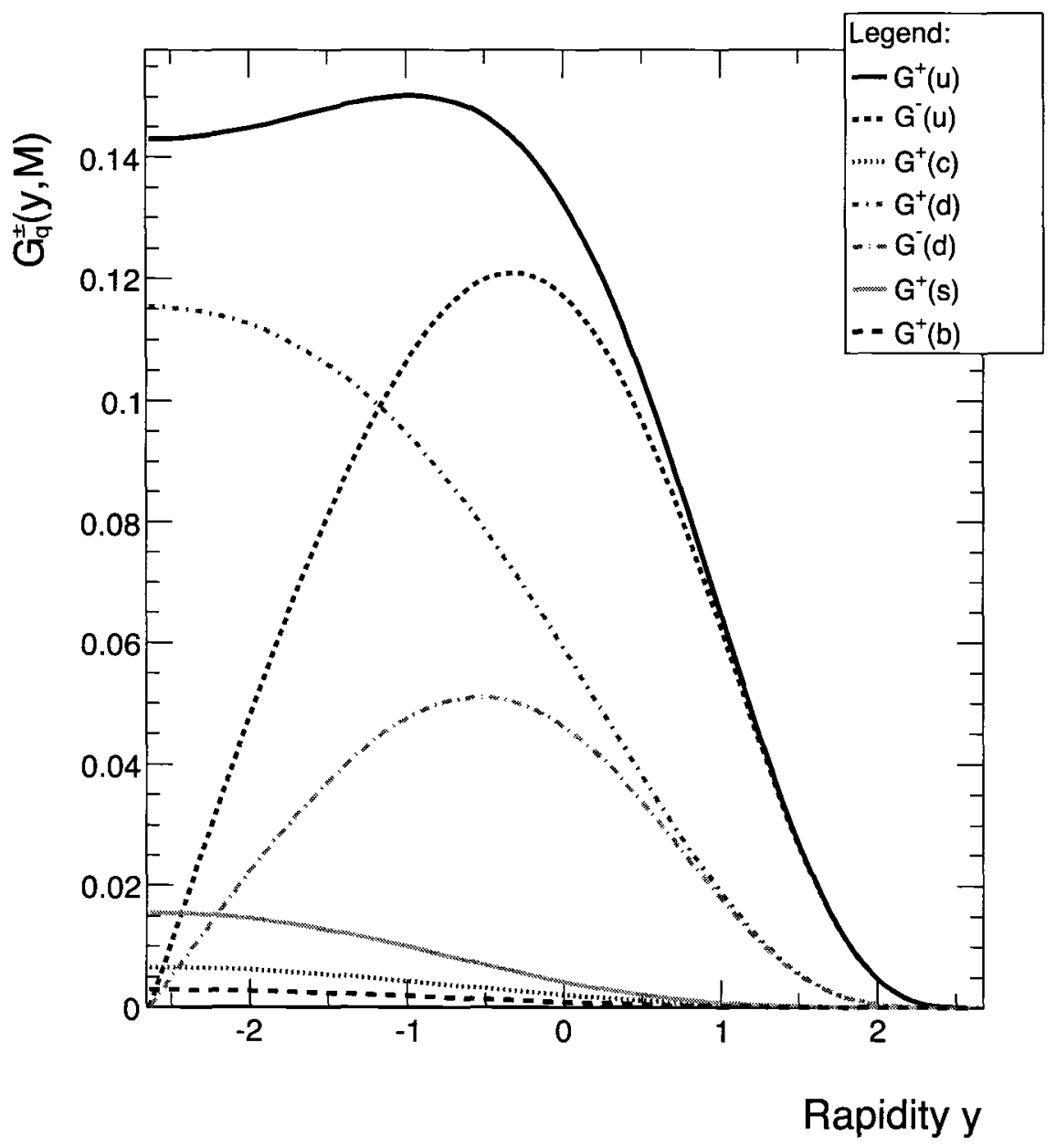

Figure 4.2: $G_{q}^{ \pm}(y, M)$ vs rapidity at energy scale $M_{q \bar{q}}=1 \mathrm{TeV}$ for $u, d, s, c$, and $b$ partons. The median of the down quark distribution occurs at a more central value of $y$ than the median for the up quark. The antisymmetric distributions occur only for up and down quarks, as an interaction between valence and sea quarks. 
there may be sensitivity to specific couplings. He considers the ratio:

$$
\begin{aligned}
R_{f f^{\prime} V} & =\frac{\sigma\left(p p \rightarrow Z^{\prime} \rightarrow f f^{\prime} V\right)}{\sigma\left(p p \rightarrow Z^{\prime} \rightarrow l^{+} l^{-}\right)}=\frac{\sigma\left(p p \rightarrow Z^{\prime}\right) B R\left(Z^{\prime} \rightarrow f f^{\prime} V\right)}{\sigma\left(p p \rightarrow Z^{\prime}\right) B R\left(Z^{\prime} \rightarrow l^{+} l^{-}\right)} \\
& =\frac{B R\left(Z^{\prime} \rightarrow f f^{\prime} V\right)}{B R\left(Z^{\prime} \rightarrow l^{+} l^{-}\right)}
\end{aligned}
$$

This cancellation is consistent when considering production within a small region about the $Z^{\prime}$ mass, where the $\mathrm{EW}$ backgrounds are negligible compared to the $Z^{\prime}$ signal. In general, $V=Z, W^{ \pm}$, resulting in the possibilities of $f f^{\prime}=l^{+} l^{-}, l^{ \pm} \nu, \nu \bar{\nu}$. However, in the analysis, Rizzo found that the bremsstrahlung of a $Z$ from a muon was insensitive to the couplings of the $Z^{\prime}$. Alternatively, a $Z$ bremsstrahlung off of a neutrino is sensitive to the neutrino couplings of the $Z^{\prime}$. In this way, neutrino final states can be distinguished, as the signal in the detector would appear to be collimated dileptons that reconstruct to a $Z^{0}$ invariant mass, plus massive missing $p_{T}$.

For a $W$ radiating off of one of the final states, Rizzo found that the resulting ratio was sensitive to the left handed lepton couplings, as the $W$ boson couples only to left handed fermions. If the $Z^{\prime}$ couples to neutrinos in a similar left handed fashion as the $\operatorname{SM} Z$, then the resulting ratio is again sensitive to the neutrino couplings. The resulting ratio for both signals is given by:

$$
\begin{aligned}
& R_{\nu \nu Z}=K_{Z} \frac{g_{L}^{\nu 2}}{\left(g_{L}^{l 2}+g_{R}^{l 2}\right)} \\
& R_{l \nu W}=K_{W} \frac{g_{L}^{\nu 2}}{\left(g_{L}^{l 2}+g_{R}^{l 2}\right)}
\end{aligned}
$$

where $K_{Z}$ and $K_{W}$ are constants, independent of the $Z^{\prime}$ couplings. In this form, it is implicitly assumed that there is no right handed neutrino.

Since these observables are dependent only on the final state couplings, they are more distinctly sensitive to the nature of the $Z^{\prime}$. However, the branching fractions 
for this signal are expected to be quite small. The result is that the loss of statistics at higher $Z^{\prime}$ masses makes them potentially ineffective at the LHC without an increase in luminosity.

\section{Associated Production}

Alternatively, Langacker, del Aguila and Cvetič ${ }^{5}$ considered Associated Production, where a $Z^{\prime}$ is produced in association with another vector boson. By considering a ratio of associated production with standard dilepton production, they found a sensitivity to the initial state up and down quarks. The ratio they consider is given by:

$$
R_{Z^{\prime} V}=\frac{\sigma\left(p p \rightarrow Z^{\prime} V\right) B R\left(Z^{\prime} \rightarrow l^{+} l^{-}\right)}{\sigma\left(p p \rightarrow Z^{\prime}\right) B R\left(Z^{\prime} \rightarrow l^{+} l^{-}\right)}
$$

Where $V=\gamma, Z, W$, in general.

It is clear from this that the final state dependence cancels out. The associated production re-weights the dependence of the interaction on the couplings to the initial state partons. In the case of an associated photon, the dependence on parton $q$ becomes $Q_{q}\left(g_{L}^{q 2}+g_{R}^{q 2}\right)$, for electric charge $Q_{q}$. Comparatively, the up quark contribution would then be weighted four times that of the down quark contribution. Since up quarks are the most likely parton to interact, this makes this ratio highly sensitive to the up quark contributions.

Additionally, the case of the production of an associated $W$ can be considered. The re-weighting results in a dependence only on the left handed coupling to the initial state partons. In some models, the $Z^{\prime}$ is entirely left handed, and should produce a much larger ratio than for other models where the $Z^{\prime}$ couples to both chiralities.

However, the statistics in the case of associated production diminish rapidly for higher $M_{Z^{\prime}}$. As a result, these three ratios are ineffective for a potentially large 
mass extra neutral gauge boson, at luminosities of $100 \mathrm{fb}^{-1}$. Other observables that are measurable for large masses will be important to further distinguish models.

\section{2 $Z^{\prime}$ Decays to Heavy Particles}

Quark jet final states are typically not considered for discovery for EW processes due to significantly larger backgrounds. Final states of gluons and light up- and downtype quarks appear as jets of hadrons in the ATLAS and CMS detectors. They can be distinguished from lepton final states but cannot be distinguished from each other. As a result, the dijet signal is swamped by the backgrounds shown in Figures 4.3 and 4.4 .

Heavy quarks, such as top and bottom quarks, are distinguishable from simple jets and result in the consideration of fewer background processes - those shown only in Figure 4.3. Bottom quarks can be distinguished in the detector as a displaced vertex, because they decay some distance from the initial interaction point. This secondary vertex can be distinguished by the high precision silicon detectors in the ATLAS $^{59}$ and CMS $^{60}$ experiments. Top quark detection comes as a consequence of bottom quark detection - top quarks are very unstable and primarily decay directly to a $W$ and a $b$-quark. Reconstruction of a $b$-quark and a $W$ boson is then required for top tagging. As a result, bottom and top quarks can be uniquely identified as final states and present an interesting method of analysis for understanding the $Z^{\prime}$.

The efficiencies involved in tagging and reconstruction will be discussed further in Section 4.4. A more detailed description of the backgrounds will be included in the final section of this chapter. 

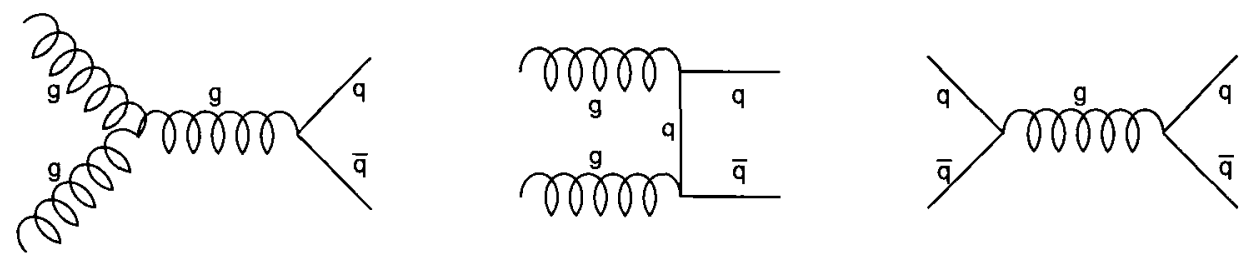

Figure 4.3: Primary SM QCD backgrounds for heavy quark production, assuming that the final state quarks can be distinguished from the general dijet background.
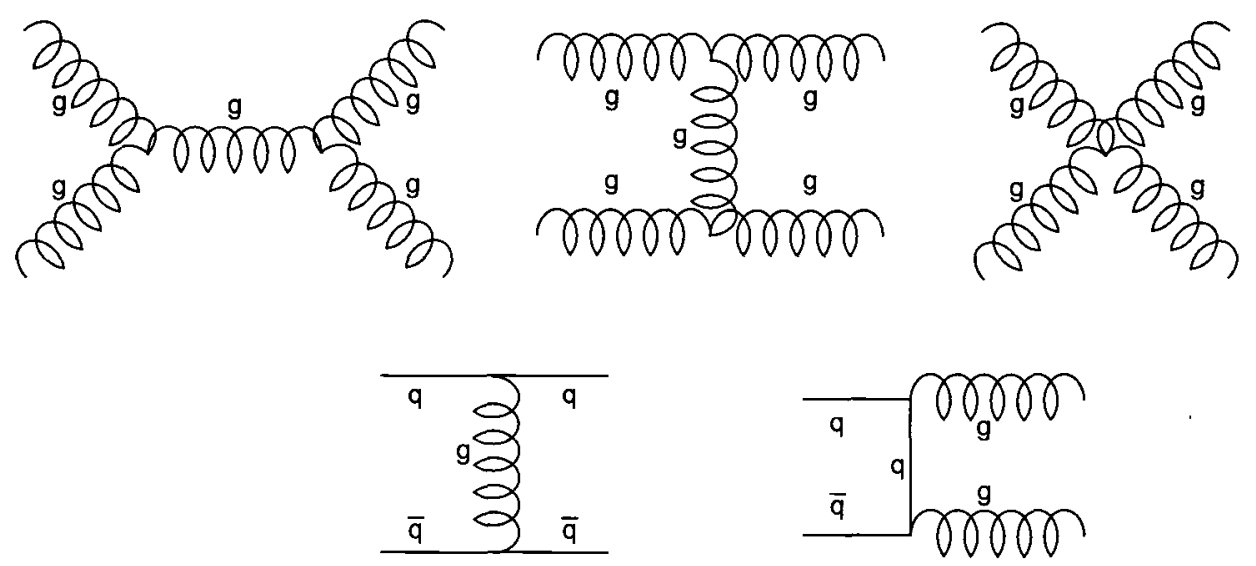

Figure 4.4: Other primary contributions to QCD backgrounds for dijet production, where quark/gluon final states cannot be distinguished from each other, not including crossing diagrams. 
An analysis of $t$ - and $b$-quark final states directly depends on the coupling strengths. Specifically, I prescribe an analysis of a ratio of the heavy quark cross sections to the muon cross section as an effective method for distinguishing between models.

For this analysis, the following ratios are defined:

$$
\begin{aligned}
R_{b / \mu} & \equiv \frac{\sigma\left(p p \rightarrow Z^{\prime} \rightarrow b \bar{b}\right)}{\sigma\left(p p \rightarrow Z^{\prime} \rightarrow \mu^{+} \mu^{-}\right)} \approx \frac{B R\left(Z^{\prime} \rightarrow b \bar{b}\right)}{B R\left(Z^{\prime} \rightarrow \mu^{+} \mu^{-}\right)}=\frac{3 K_{q}\left(g_{L}^{b 2}+g_{R}^{b 2}\right)}{\left(g_{L}^{\mu 2}+g_{R}^{\mu 2}\right)} \\
R_{t / \mu} & \equiv \frac{\sigma\left(p p \rightarrow Z^{\prime} \rightarrow t \bar{t}\right)}{\sigma\left(p p \rightarrow Z^{\prime} \rightarrow \mu^{+} \mu^{-}\right)} \approx \frac{B R\left(Z^{\prime} \rightarrow t \bar{t}\right)}{B R\left(Z^{\prime} \rightarrow \mu^{+} \mu^{-}\right)}=\frac{3 K_{q}\left(g_{L}^{t 2}+g_{R}^{t 2}\right)}{\left(g_{L}^{\mu 2}+g_{R}^{\mu 2}\right)}
\end{aligned}
$$

where $K_{q}$ is a constant depending on the QCD and EW correction factors, and the factor of 3 is due to summation over color final states. It is apparent that these ratios depend on only four couplings from each model.

An analysis based on the location of a measured $Z^{\prime}$ in the parameter space of $R_{b / \mu}$ vs $R_{t / \mu}$ presents a highly model dependent observation for distinguishing between models. The primary difficulty in this analysis will be distinguishing the signal from the large QCD background for the quark final states.

\subsection{Identifying Generation Universality}

There are a few models that allow for a $Z^{\prime}$ that does not couple universally to each generation, including various Topcolor models, anomaly free little Higgs models, models that take the form of $S U(2)_{h} \times S U(2)_{l}$, and models with the form $U(1)_{h} \times U(1)_{l}$. However, if a model independent study is to be performed for a $Z^{\prime}$, I believe that this property should be examined.

This is a trivial calculation that involves the comparison of cross sections for third generation particles to those of other generations within the same family. 
Experimental complications arise for this measurement in the quark families. The ATLAS TDR ${ }^{59}$ suggests that charm final states may be distinguishable, which would allow a ratio of third to second generation quark cross sections. Leptonic channels present less of a challenge since $\tau$ final states are distinguishable and $e$ and $\mu$ final states have high tagging efficiencies.

With these final states, it is possible to consider the following ratios to examine generation universality:

$$
\begin{aligned}
R_{t / c} & =\frac{\sigma\left(p p \rightarrow Z^{\prime} \rightarrow t \bar{t}\right)}{\sigma\left(p p \rightarrow Z^{\prime} \rightarrow c \bar{c}\right)} \\
R_{\tau / \mu} & =\frac{\sigma\left(p p \rightarrow Z^{\prime} \rightarrow \tau^{+} \tau^{-}\right)}{\sigma\left(p p \rightarrow Z^{\prime} \rightarrow \mu^{+} \mu^{-}\right)} \\
R_{\tau / e} & =\frac{\sigma\left(p p \rightarrow Z^{\prime} \rightarrow \tau^{+} \tau^{-}\right)}{\sigma\left(p p \rightarrow Z^{\prime} \rightarrow e^{+} e^{-}\right)}
\end{aligned}
$$

Each of these are important ratios to be considered for a general analysis of the nature of the $Z^{\prime}$. For most models, those that have universal couplings between the $Z^{\prime}$ and the SM fermions, these ratios should be equal to 1 . However, in the case of a low mass $Z^{\prime}$, the branching ratio for the top quark is reduced $\left(\beta_{t}<1\right)$, and the mass term becomes non-negligable $\left(\frac{4 m_{t}^{2}}{M_{Z^{\prime}}^{2}} \approx 0.12\right.$ at $\left.M_{Z^{\prime}}=1000 \mathrm{GeV}\right)$. This would result in a measurement of $R_{t / c}<1$ for a model with generation universality.

Additionally, the hadronic ratio suffers from low tagging efficiencies for both final state measurements. As a result, this measurement will suffer from large uncertainties and will only prove useful in the cases of leptophobic models and where there is generation universality within the lepton sector (such as Anomaly Free SLH). As such, this calculation is not included in Chapter 5. The $\tau$ to $e$ ratio does not provide any useful information that is not already provided by the ratio of third to second generation leptons - which has better statistics - and is also not considered in the following Chapter. 
The primary difficulties in this measurement will be the tagging efficiencies for $t$-quark and $\tau$-lepton final states. Luminosity becomes a significant factor for models without generation universality, as the first two generations are suppressed. The production cross section is then reduced due to the interacting first and second generation partons and results in a need for an increased luminosity for adequate statistics.

\subsection{Parton Distributions, Backgrounds and Effi- ciencies}

\subsubsection{Parton Distribution Functions}

There are a variety of packages available for numerical results for the parton distribution functions. These include, but are not limited to, the CTEQ ${ }^{61}$ collaboration's and MRST ${ }^{62}$ collaboration's packages. The CTEQ (Coordinated Theoretical Experimental Project on QCD) package is used for all my calculations; specifically, the CTEQ6M table is used, which uses the standard $\overline{M S}$ renormalization scheme, with $\alpha_{s}=0.118 .^{61}$

\subsubsection{Detector Efficiencies}

While the ATLAS experiment will employ recent advances in detector technology, it will not be able to distinguish final states with $100 \%$ efficiency. Specifically, the SM fermions that are expected final states from $Z^{\prime}$ production will have efficiencies that vary based on flavour and kinematics. The minimum requirement for detection of any 
particle is $p_{T}>0.5 \mathrm{GeV}$, and particle tracking only occurs within the pseudo-rapidity range of $|\eta|<2.5$. Outside of this range, the end caps are unable to track the path of the particles, and act simply as energy calorimeters.

The ability to detect and distinguish the final states is critical for the analysis presented. Currently, both $\mathrm{CMS}^{60}$ and ATLAS ${ }^{59}$ have presented estimates of the efficiency for distinguishing these particles, but these are based on simulation and will be updated with calibration and initial testing. Since these are simply estimates, I include two possibilities - one "optimistic" and one "pessimistic" - for tagging efficiencies based on factors considered below.

\section{$t$-Quark Events}

Top quarks decay rapidly, long before they are able to reach the detectors. Instead, they decay primarily to a bottom quark and a $W$ boson, as they are above the $W b$ threshold, which each then decay further. The $W$ decays approximately $65 \%$ to $j j$, and another $35 \%$ to $l \nu$. The detection of a top quark then depends on the reconstruction from these final states. For this reason, there are several primary modes that can be considered: fully hadronic $(t \bar{t} \rightarrow b \bar{b} j j j j)$, semileptonic $(t \bar{t} \rightarrow$ $\left.b \bar{b} j j l^{-} \bar{\nu}\left(l^{+} \nu\right)\right)$ and fully leptonic $\left(t \bar{t} \rightarrow b \bar{b} l^{+} \nu l^{-} \bar{\nu}\right)$. The most distinct signal is the semileptonic mode with $e$ and $\mu$ leptons ( $\tau$ neglected due to reconstruction required), which presents the best signal to background ratio, and accounts for $29.6 \%$ of all $t \bar{t}$ decays.

Considering $p_{T}$ cuts and analysis of the jets and lepton and missing $E_{T}$, approximately $14 \%$ of these events can be distinguished (as stemming from top quark decays) from interactions that produce similar final states, at the ATLAS experiment. 
Combining with the branching ratio, this results in an expected efficiency of $4 \%$ for the semileptonic mode. Considering the other modes, the total efficiency in detecting and reconstructing $t \bar{t}$ events could be as high as $10 \%$. Alternatively, these estimates could be high, so I further consider the "pessimistic" scenario of $1 \%$ efficiency, as a Devil's Advocate situation.

As a final note: limits on the $Z^{\prime}$ for certain models place expectations for the mass in the $\mathrm{TeV}$ range. Such high energy decays will result in highly boosted final states and highly collimated jets. At such high energies, there is some concern ${ }^{63-65}$ over the ability to distinguish the decay products from top quark final states. Primarily, top final states have been considered in the literature for $M_{t \bar{t}}<1 \mathrm{TeV},{ }^{64}$ which may not be sufficient for the analysis of a high mass $Z^{\prime}$. Though estimates on actual detection efficiencies are not yet determined, these new studies do conclude that detection/analysis is possible with different tagging methods. Since these cannot yet be quantified, I employ the tagging efficiencies valid at lower energies for higher mass resonances.

\section{b-Quark Events}

Bottom quarks are distinguished by the observation of a secondary vertex some distance from the initial interaction. The ATLAS TDR suggests that an efficiency of $50 \%$ is a good estimate for distinguishing a single $b$-quark from light jet backgrounds at high luminosities* The fake rate for this scenario is estimated to be $1 \%$. This

"If it is possible to consider the scenario where a single $b$-jet implies that the "other" jet is also from a $b$-quark, then it may be possible to consider an effective efficiency $>50 \%$ for $b \bar{b}$ events. If we assume that $\sim 50 \%$ of the events are tagged by the $b$, it may be that approximately $50 \%$ of the remaining events can be tagged by the $\bar{b}$. However, there may be kinematic and systematic reasons 
efficiency is assumed in the scenario that the distinction of a single $b$ jet implies that the second jet is also a $b$ jet. Additionally, I consider the scenario of requiring the simultaneous tagging of both jets as $b$-jets, which results in a reduced efficiency of $25 \%$ and a negligible fake rate.

The tagging efficiency in the case of highly boosted final states is the same as for light jets, as the jet kinematics hides the secondary vertex. In this case, kinematic or soft lepton tagging may be effective alternate methods. However, this is beyond the scope of consideration and is left to be determined if the situation arises.

\section{$\tau$-Lepton Events}

Similar to the top quark, the $\tau$ decays via a virtual $W$ inside the detector. As a result, the same modes can be considered: fully hadronic $\left(\tau^{+} \tau^{-} \rightarrow \nu_{\tau} \bar{\nu}_{\tau} j j j j\right)$, semileptonic $\left(\tau^{+} \tau^{-} \rightarrow \nu_{\tau} \bar{\nu}_{\tau} j j l \nu\right)$ and fully leptonic $\left(\tau^{+} \tau^{-} \rightarrow \nu_{\tau} \bar{\nu}_{\tau} l \nu l \nu\right)$. The semileptonic mode is again the most distinct mode and occurs approximately $45 \%$ of the time, with $75 \%$ of these events containing $\geq 1 \pi^{0}$ and exactly 1 charged pion (one-prong decays).

A hadronic mode, considered by Anderson, et $a l,{ }^{66}$ with one $\tau$ decaying to a $\pi$ and the other to jets, results in their estimation of a $6 \%$ effective usable efficiency based on kinematics, branching ratios, acceptance and efficiencies. However, ATLAS and $\mathrm{CMS}^{67}$ consider the fully hadronic mode by considering jet clusters as candidates for $\tau$ jets. ATLAS estimates a $40 \%$ efficiency for distinguishing $\tau$ jets from QCD backgrounds, which results in approximately $6.8 \%$ of di-tau events from the decay of a $Z^{\prime}$. Alternatively CMS suggests a $\sim 70 \%$ efficiency, resulting in $\sim 21 \%$ of events, but has a lower rejection rate for QCD backgrounds.

why the efficiency of distinguishing events from the remainder is less than $50 \%$. The use of the $50 \%$ is a representative number intended to describe an optimistic scenario. 
A more pessimistic scenario arises from considering the results from the Tevatron. $\mathrm{CDF}^{68}$ and $\mathrm{D} 0^{69}$ results for the number of $\tau^{+} \tau^{-}$events observed in $Z^{\prime}$ decays can be considered against the expectation from $\sigma \times B R\left(Z \rightarrow \tau^{+} \tau^{-}\right) \times L$. From this, the effective efficiency from D0 appears to be $\sim 1.5 \%$, while that from CDF is $\sim 0.4 \%$.

Considering these scenarios, I employ efficiencies of $20 \%$ and $1 \%$ in my analysis for $\tau^{+} \tau^{-}$pair production as optimistic and pessimistic cases, respectively.

\section{Other Considerations}

The ability to observe electrons, muons and charm quarks will also be useful in the analysis. The ATLAS TDR lists efficiencies for these as shown in Table 4.2.

Table 4.2: Detection efficiencies for $e, \mu$ leptons, and $c$ jets for ATLAS. Lepton efficiencies based on $p_{T_{\ell}}=1 \mathrm{GeV}$ and $200 \mathrm{GeV}$ events, respectively.

\begin{tabular}{|c|c|c|}
\hline Particle & Pessimistic & Optimistic \\
\hline \hline -lepton & $>75 \%$ & $>90 \%$ \\
$\mu$-lepton & $>96 \%$ & $>98 \%$ \\
\hline$c$-jet & $10 \%$ & $10 \%$ \\
\hline
\end{tabular}

\subsubsection{Backgrounds and Tagging}

The primary backgrounds involving leptonic final states are Electroweak. The DrellYan production of $\gamma$ and $Z$ are specifically considered. These backgrounds are also involved in the production of quark final states, however they are orders of magnitude smaller than the QCD backgrounds. Electroweak backgrounds are accounted for during the calculation of the $Z^{\prime}$ interactions, including interference. QCD backgrounds are calculated via the WHiZard (W, Higgs, Z and Related Decays) program, ${ }^{70}$ 
which is a generic Monte-Carlo event generator that analyzes arbitrary multi-particle processes. WHiZard can employ the O'Mega, CompHep and/or MadGraph matrix element generators, though O'Mega was used for the backgrounds calculated within this thesis. Only SM processes are taken into account for backgrounds.

QCD backgrounds provide a significant challenge for measurements of electroweak decays to quark final states, with the most prominent backgrounds shown in Figure 4.3 for heavy quark production, and shown in Figure 4.4 for general dijet production. However, these backgrounds can be reduced using cuts and non-standard tagging methods. ${ }^{63-65}$

In all cases, $q \bar{q}$ annihilation through a $Z^{\prime}$ produces final states that have, on average, a high $p_{T}$, while QCD backgrounds have a much lower $p_{T_{q}}$, on average. For this reason, I propose high $p_{T}$ cuts on final states to improve the signal to background ratio. To reduce the background, I apply a cut of $p_{T_{t}} \geq 0.3 M_{Z^{\prime}}$ to each final state top/bottom. For top quark final states, this is not always a directly applicable cut, but is meant to test the feasibility of the measurement. It remains to be determined what cuts on the decay products of the top adequately represent this $p_{T}$ cut.

Additionally, a tight invariant mass window of $0.965 M_{Z^{\prime}} \leq M_{f \bar{f}} \leq 1.035 M_{Z^{\prime}}$ is applied for the analysis of quark final states in order to reduce the number of background events included. While reducing backgrounds significantly, this also limits the statistics. The window was chosen as a balance between total signal and the signal to background ratio. When leptonic final states are considered, this window is relaxed to $0.93 M_{Z^{\prime}} \leq M_{f \bar{f}} \leq 1.07 M_{Z^{\prime}}$.

In general, lepton and jet energy resolution in the detectors results in a smearing effect - the events within the peak region appear to be spread out, decreasing 
the magnitude of the peak and increasing the apparent width. This could potentially affect signal statistics in the cut window. A smearing effect would result in reduced signal statistics while having a minor effect on backgrounds. Ultimately, this would result in an increased uncertainty in measurements. However, this was not taken into account in this thesis. 


\section{Chapter 5}

\section{Results}

\subsection{Calculation Methods}

The following results were calculated using a combination of techniques. Primarily, the $Z^{\prime}$ signal was calculated using a Monte-Carlo integration technique with weighted events, applied to Equation (4.11). Kinematic cuts were implemented within the integration. EW backgrounds were included in the calculation of the $Z^{\prime}$ signal to account for interference, while QCD backgrounds were calculated using weighted event generation for SM processes using WHiZard package ${ }^{70}$ with O'Mega matrix element generation. Uncertainties considered are purely statistical, arising from the number of binned events. 


\subsection{Discovery potential at the LHC}

The ability of the LHC to discover a $Z^{\prime}$ is not in question - one of the easiest measurements to make at the LHC will be that of a non-leptophobic $Z^{\prime}$ within a kinematically accessible mass range. The invariant mass distributions for ideal detection scenarios are described in Figure 5.1, where several recent and popular models are explored. The total event rate is then found via the relation $N_{\mu^{+} \mu^{-}}=L \times \frac{d \sigma}{d M}$, for luminosity L.

Figure 5.1 clearly shows the trend of increase in width of the $Z^{\prime}$ and decrease in both signal and background for larger mass ranges. Smearing effects in detectors would amplify the trend of increasing width, further compounding the issue. Of note is the noticeable effect of interference with the $Z$ and $\gamma$ for most models, which suggests that the discovery of a high mass $Z^{\prime}$ could occur via observation of missing dilepton cross section at very high luminosities. Hypothetically, if the resonance peak is just outside of a kinematically accessible range, the effect of the interference between the $Z^{\prime}$ and the SM neutral gauge bosons may be measurable with high integrated luminosities.

Considering the production of muon pairs, Figures 5.2 and 5.3 show the integrated luminosity required to detect a resonance of a $Z^{\prime}$ for each model considered with the application of an invariant mass window of $0.93 M_{Z^{\prime}} \leq M_{\ell^{+} \ell^{-}} \leq 1.07 M_{Z^{\prime}}$ and a requirement of 10 events for detection. A $Z^{\prime}$ should be discoverable within the first few months of the LHC running at full energy for $M_{Z^{\prime}} \leq 2 \mathrm{TeV}$, depending on the model and assuming that the initial year of running will produce an integrated luminosity of approximately $10 \mathrm{fb}^{-1}$.

Since the dimuon channel includes vertices of only first and second generation 


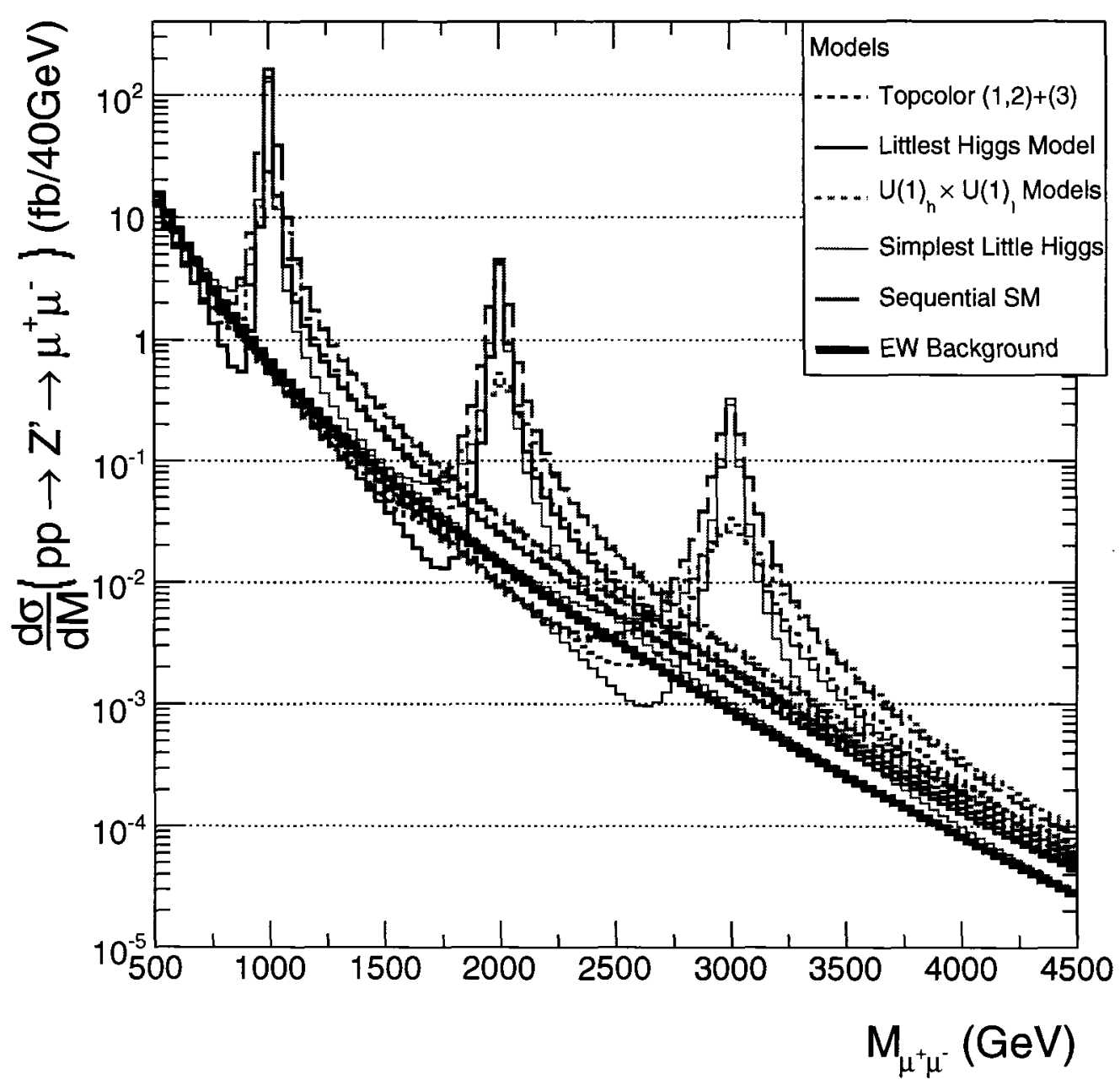

Figure 5.1: Invariant mass distribution for $M_{Z^{\prime}}=1,2$ and $3 \mathrm{TeV}$, for a representative set of models, illustrating the decrease in signal for larger mass ranges. The background is displayed in prominent solid black. The effects of interference with the $Z^{0}$ and $\gamma$ are visibly noticeable for most models.

fermions, the production of a $Z^{\prime}$ for models with preferential couplings to third generation fermions will take significantly longer to observe. The event rate is dropped by a factor of the preferential coupling to the fourth power (i.e.: $\tan \phi^{-4}$ in the $S U(2)_{h} \times S U(2)_{l}$ models) over a similar interaction with a third generation fermion. Figure 5.4 shows the $\tau^{+} \tau^{-}$invariant mass distribution for the same models, highlighting the significantly increased cross section for both the Topcolor and $U(1)_{h} \times U(1)_{l}$ models. A similar result can be expected for the $S U(2)_{h} \times S U(2)_{l}$ models. 


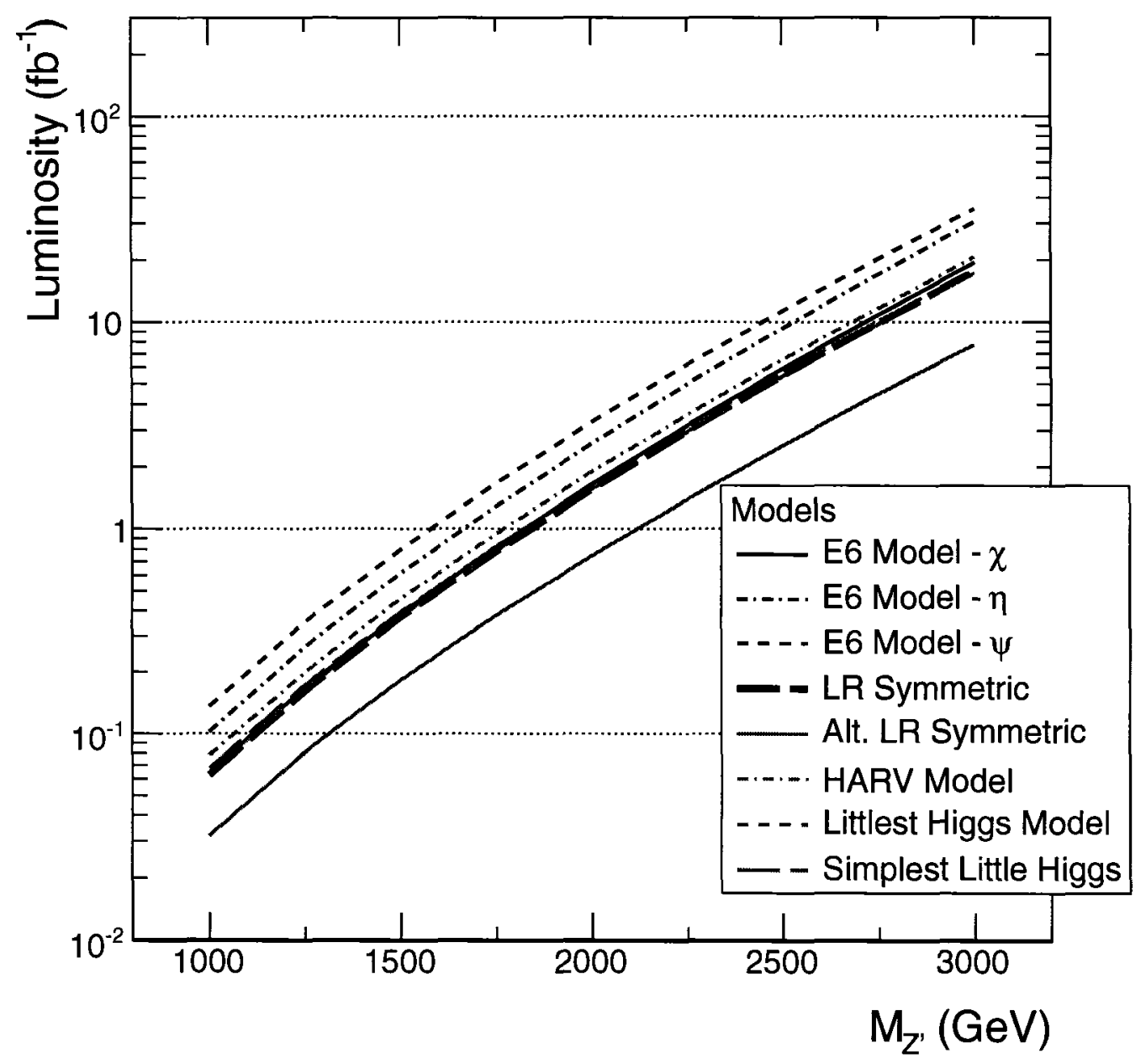

Figure 5.2: Luminosity required for the discovery of a $Z^{\prime}$ in the dimuon channel, assuming a requirement of 10 events, for a mass range of $1 \mathrm{TeV} \leq M_{Z^{\prime}} \leq 3 \mathrm{TeV}$. In each case, $S / \sqrt{B}>5$. Models include the majority of those with generation universality.

Should one of these models be realized in nature, the tau channel may then be the discovery mode. Figures 5.5 and 5.6 show the same luminosity plots as before, except for tau final states. From this, it is apparent that 10 events will be observable for models with preferential third generation couplings at lower luminosities in the tau channel than in the muon channel if we assume the optimistic tau tagging efficiency of $\epsilon_{\tau}=0.2$. In fact, an efficiency as low as approximately 0.06 will still result in a tau discovery for the last two models listed in Figure 5.6. For the Topcolor model considered, the optimistic tau efficiency or better will be required for discovery to 


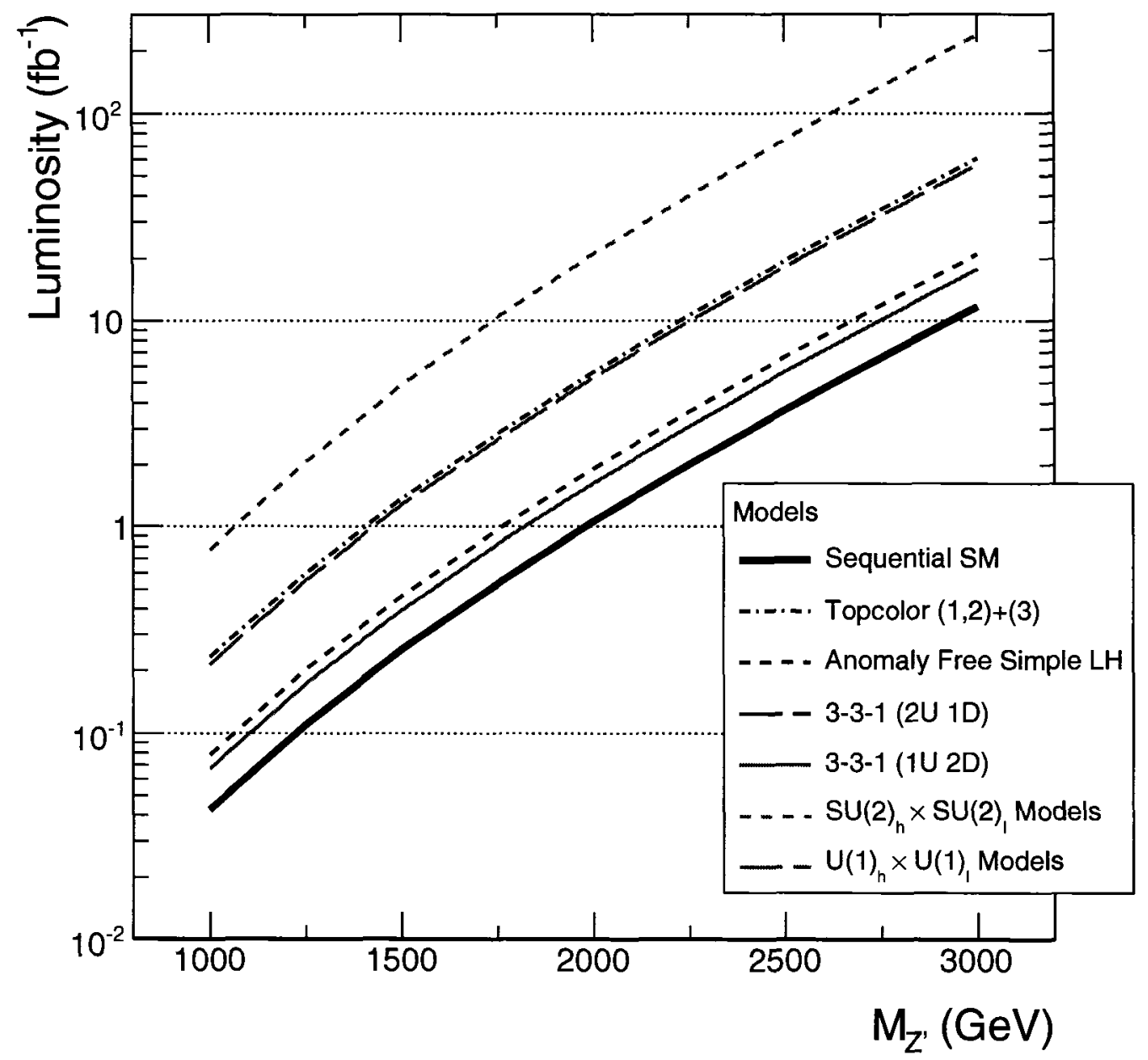

Figure 5.3: Luminosity required for the discovery of a $Z^{\prime}$ in the dimuon channel, assuming a requirement of 10 events, for a mass range of $1 \mathrm{TeV} \leq M_{Z^{\prime}} \leq 3 \mathrm{TeV}$. In each case, $S / \sqrt{B}>5$. Models include primarily those without generation universality. occur in the tau channel.

Table 5.1 presents the $S / \sqrt{B}$ (signal over square root of background) ratio for the detection of 10 events. From this, it is obvious that there is $<1$ background event expected at these low luminosities, so the detection of a number of dilepton events in these high energy ranges at these low luminosities will be an explicit discovery of something beyond the SM, regardless of the nature of BSM.

Though discovery should be an early success of the LHC, the determination of the nature of the $Z^{\prime}$ will require higher luminosities. Additionally, the luminosity needed for discovery for some models, specifically those with preferential third gen- 
Table 5.1: Ratio of $\mathrm{S} / \sqrt{\mathrm{B}}$ at integrated luminosity required to produce 10 events, for 1,2 and $3 \mathrm{TeV} Z^{\prime}$ resonances, assuming invariant mass windowing as defined at the beginning of this section.

\begin{tabular}{|l|c|c|c|}
\hline Model & $M_{Z^{\prime}}=1 \mathrm{TeV}$ & $M_{Z^{\prime}}=2 \mathrm{TeV}$ & $M_{Z^{\prime}}=3 \mathrm{TeV}$ \\
\hline \hline E6 Model $-\chi$ & 26.0 & 25.0 & 23.6 \\
\hline E6 Model $-\eta$ & 20.7 & 19.9 & 18.8 \\
\hline E6 Model - $\psi$ & 17.9 & 17.6 & 17.5 \\
\hline LR Symmetric & 26.4 & 25.6 & 24.8 \\
\hline Alt. LR Symmetric & 37.0 & 37.1 & 37.3 \\
\hline HARV Model & 23.6 & 23.2 & 22.9 \\
\hline Sequential SM & 31.9 & 31.1 & 30.3 \\
\hline Topcolor $(1,2)+(3)$ & 13.7 & 13.5 & 13.3 \\
\hline Littlest Higgs Model & 26.1 & 25.6 & 25.1 \\
\hline KK Model & 41.8 & 40.8 & 39.7 \\
\hline Simplest Little Higgs & 25.4 & 25.1 & 24.7 \\
\hline Anomaly Free Simple LH & 23.5 & 23.1 & 22.7 \\
\hline 3-3-1 Model (2U 1D) & 31.6 & 30.8 & 30.0 \\
\hline 3-3-1 Model $(1 U$ 2D) & 25.4 & 25.1 & 24.7 \\
\hline$S U(2)_{h} \times S U(2)_{l}$ Models & 7.5 & 7.0 & 6.7 \\
\hline$U(1)_{h} \times U(1)_{l}$ Models & 14.2 & 13.9 & 13.8 \\
\hline
\end{tabular}




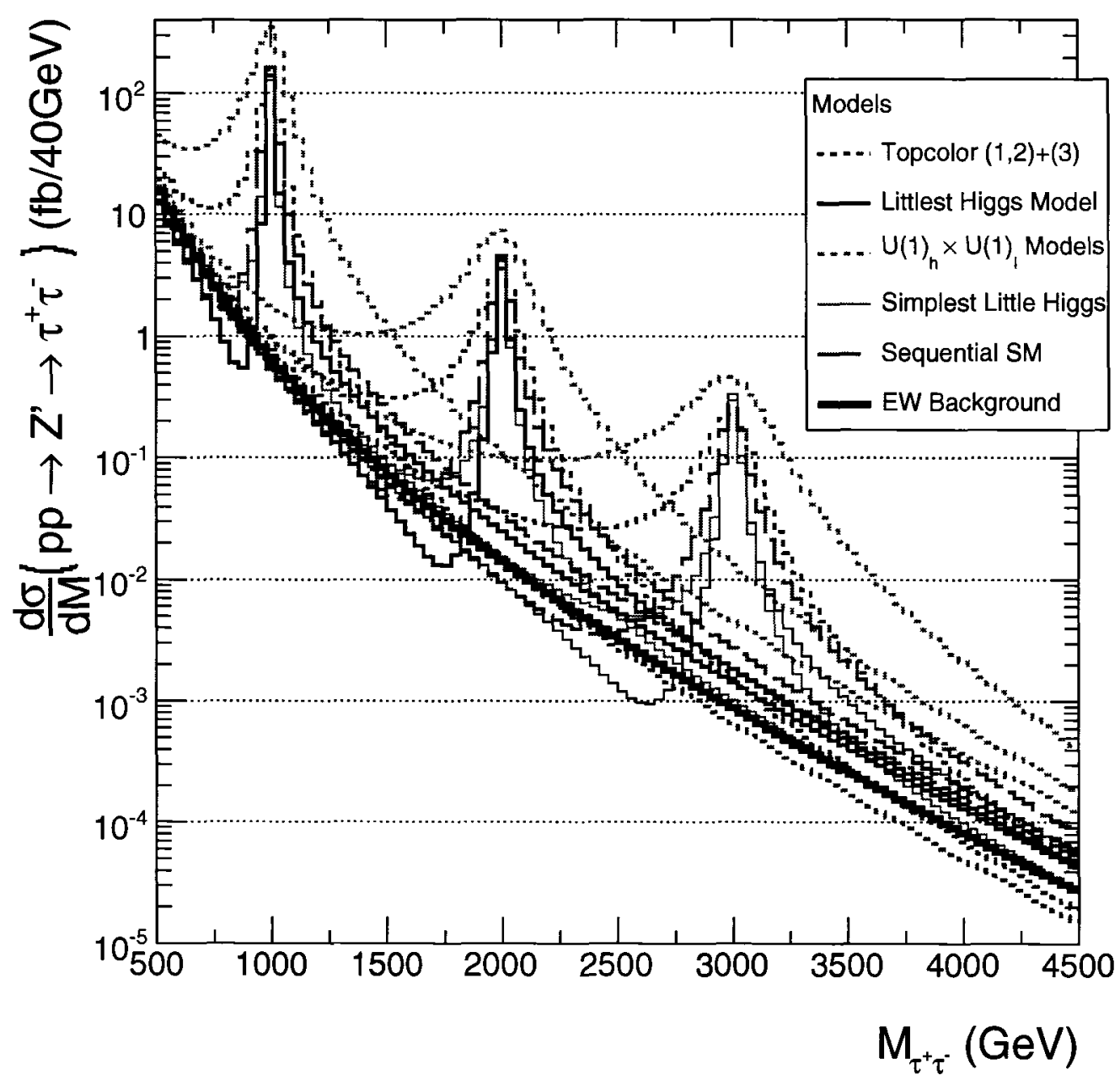

Figure 5.4: $\tau$ channel invariant mass distribution for $M_{Z^{\prime}}=1,2$ and $3 \mathrm{TeV}$, for a representative set of models, illustrating the increased cross section for models with preferential couplings to third generation fermions.

eration couplings, remains well above $10 \mathrm{fb}^{-1}$ or even $20 \mathrm{fb}^{-1}$ for masses greater than $2.25 \mathrm{TeV}$, resulting in the discovery of such a boson remaining out of reach for several years. Thus, the lack of an excess of dilepton events early on should not diminish the need for continued attention to this channel. 


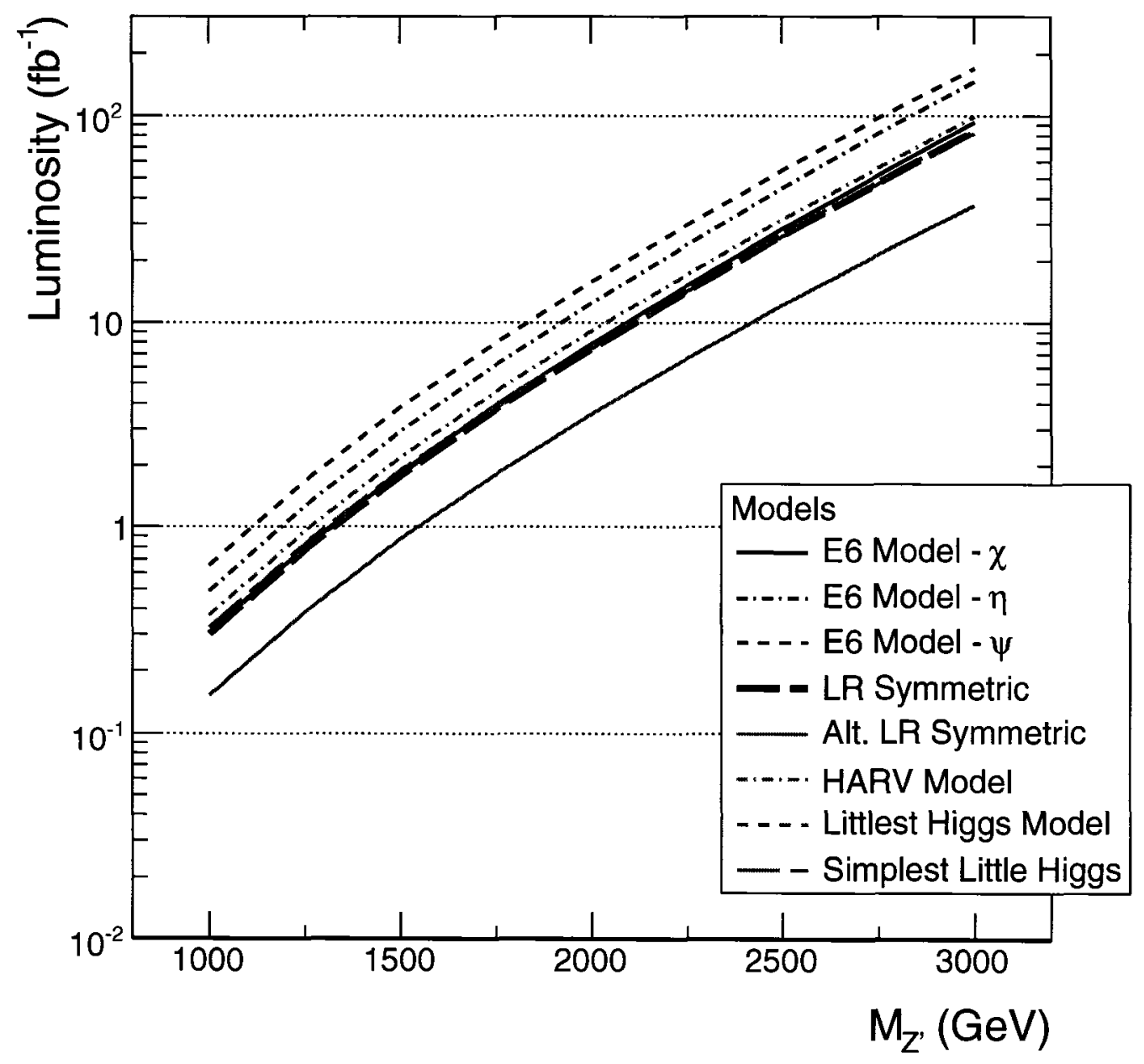

Figure 5.5: Luminosity required for the discovery of a $Z^{\prime}$ in the di-tau channel, assuming a requirement of 10 events and the optimistic efficiency $\left(\epsilon_{\tau}=0.2\right)$, for a mass range of $1 \mathrm{TeV} \leq M_{Z^{\prime}} \leq 3 \mathrm{TeV}$. In each case, $S / \sqrt{B}>5$. Models include the majority of those with generation universality. 


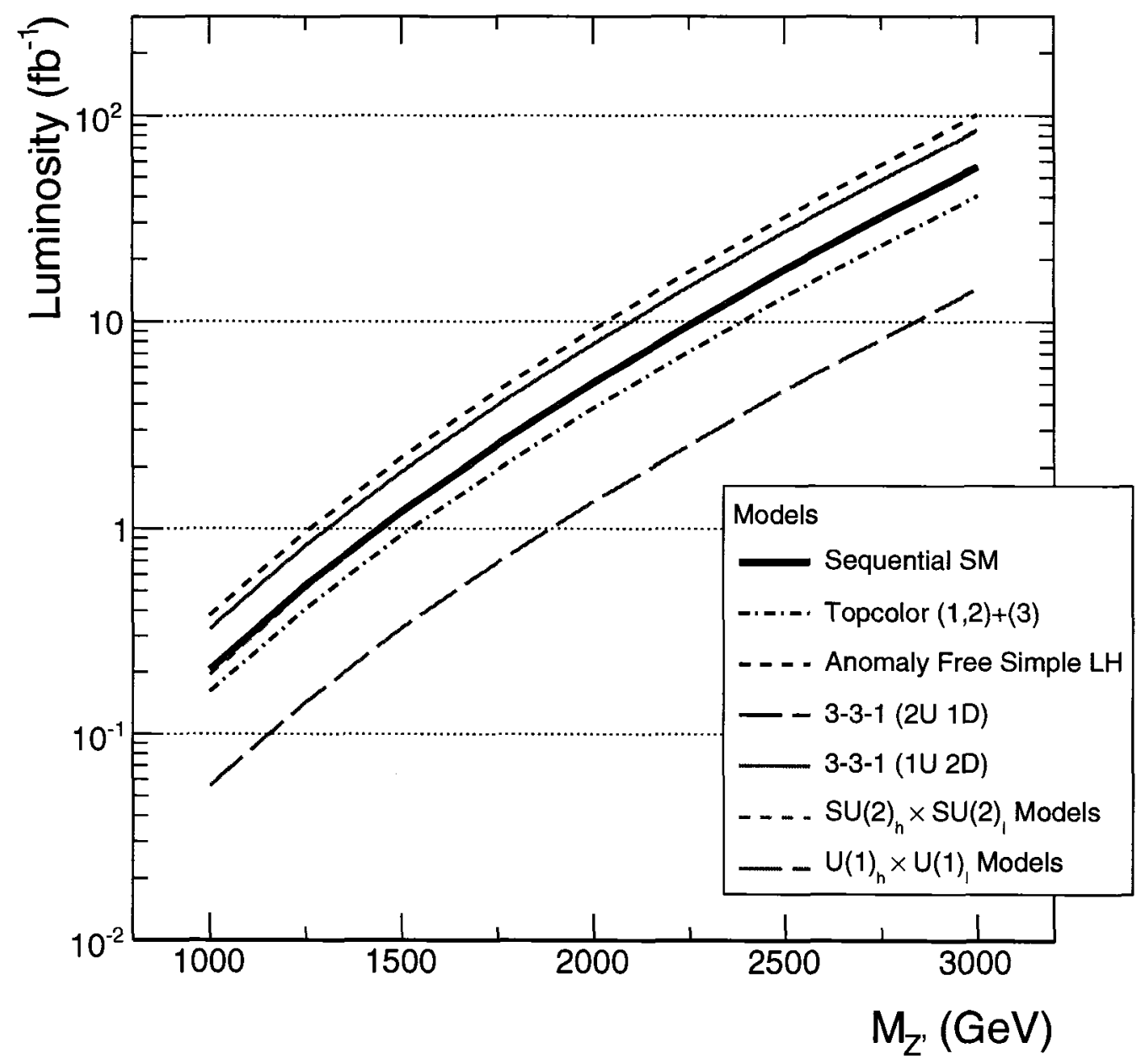

Figure 5.6: Luminosity required for the discovery of a $Z^{\prime}$ in the di-tau channel, assuming requirement of 10 events and the optimistic efficiency $\left(\epsilon_{\tau}=0.2\right)$, for a mass range of $1 \mathrm{TeV} \leq M_{Z^{\prime}} \leq 3 \mathrm{TeV}$. In each case, $S / \sqrt{B}>5$. Models include primarily those without generation universality. 


\subsection{Heavy Quark Final States}

\subsubsection{Viability of quark final state measurements}

Several issues are involved in the detection of high energy quark final states, discussed in Section 4.4.3. In my analysis, I assume that these contribute to the efficiency, and I apply kinematic cuts to the signal assuming reconstruction of the invariant mass of the events. In the ATLAS experiment, the reconstruction of the invariant mass and transverse momentum will be significantly affected by missing transverse energy (from neutrinos in $W \rightarrow l \nu$ ), $E_{T}$, which is not taken into account in this thesis. A more rigorous detector level simulation would affect results, and presents a future further step in this analysis.

Figures 5.7 and 5.8 show the invariant mass distribution before and after the application of the $p_{T_{q}}>0.3 M_{Z^{\prime}}$ cut for a $1500 \mathrm{GeV}$ and a $3000 \mathrm{GeV} Z^{\prime}$. Prior to the cut, the signal in the peak region is approximately equal in magnitude to the background with a slight excess of signal for the higher mass instance. The QCD backgrounds fall primarily as $1 / M^{4}$, while the $Z^{\prime}$ signal falls as $1 / M^{3}$. As a result, the higher mass signal is more distinguishable from the background - though both suffer from an overall decrease in number of events.

The application of the transverse momentum cut improves the signal over background at the expense of some of the signal. In addition, the primary QCD background, gluon fusion, tends to have a lower average $p_{T}$ for the same interaction energy. The optimum cut proved to be approximately $0.3 M_{Z^{\prime}}$; a stronger cut improves the signal to background ratio, but decreases the total signal and thus increases the statistical uncertainty. 


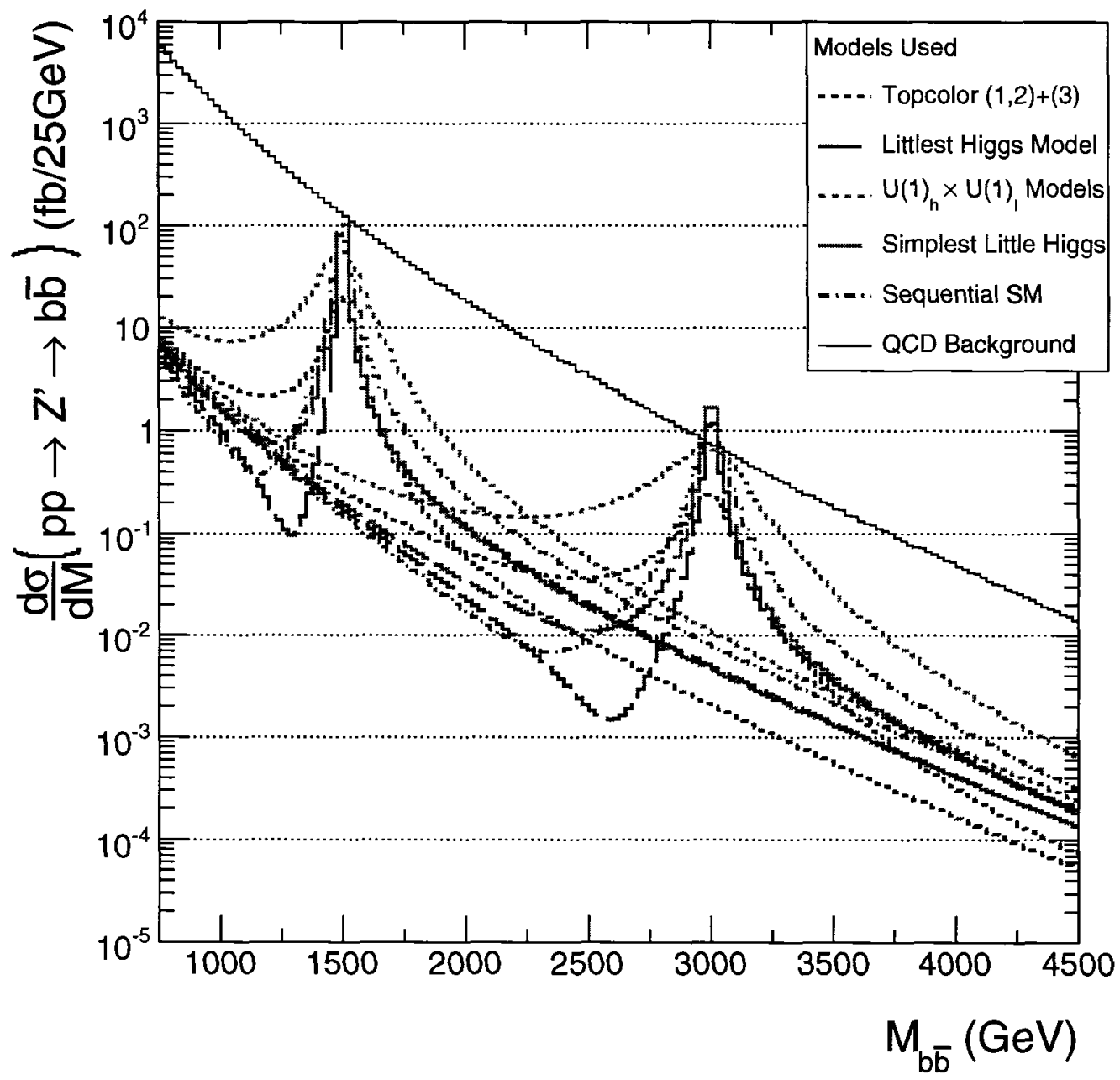

Figure 5.7: Invariant mass distribution for production of $t \bar{t}$ pairs with no kinematic cuts. QCD backgrounds are separated from BSM EW processes $\left(M_{Z^{\prime}}=\right.$ $1500,3000 \mathrm{GeV}$ ) to illustrate the relative strength of the signals. 


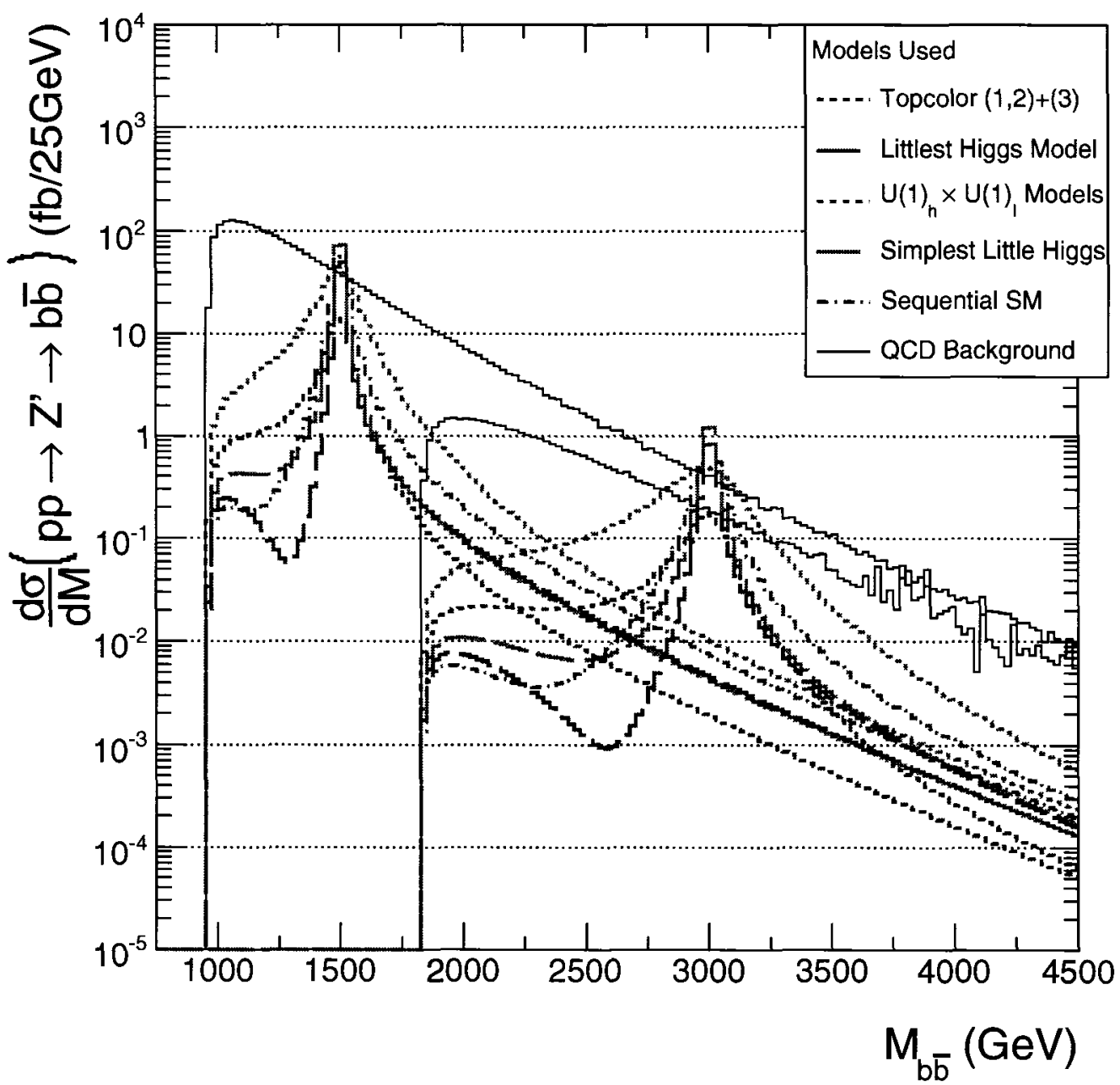

Figure 5.8: Invariant mass distribution for production of $t \bar{t}$ pairs with $p_{T_{t}}>$ $0.3 M_{Z^{\prime}}$ cuts. QCD backgrounds are separated from BSM EW processes $\left(M_{Z^{\prime}}=\right.$ $1500,3000 \mathrm{GeV}$ ) to illustrate the relative strength of the signals. 
Similarly, Figures 5.9 and 5.10 show the invariant mass distribution for the production of $b \bar{b}$ pairs from QCD and BSM EW processes. The backgrounds for $b \bar{b}$ pairs are significantly larger, especially at the low energy ranges, because the production is more kinematically favoured. Some models shown are dwarfed by the backgrounds, such as the Topcolor model, which suffers from the "seesaw" effect that prefers $t \bar{t}$ final states. However, the application of cuts affects the dominant gluon fusion backgrounds more significantly than $q \bar{q}$ annihilation, and so the signal to background is improved.

Tables 5.2 and 5.3 shows the signal to total event ratio for $b \bar{b}$ and $t \bar{t}$ pairs based on $L=10 \mathrm{fb}^{-1}$. In some cases the signal is very distinctive for both optimistic and pessimistic efficiencies, such as in the HARV model. However, other models, such as the ALRSM and Topcolor, are difficult to distinguish from general fluctuations in the signal in one of the $t \bar{t}$ or $b \bar{b}$ channels, or both. In the case of the Topcolor model, the large preferential coupling to the third generation results in a very weak interaction cross section.

While a 5-sigma signal over background is required for discovery, making accurate measurements requires as high precision as possible. The percent uncertainty in the measurements goes as the inverse of $S / \sqrt{S+B}$. In many cases, the uncertainty in the measurement will result in an inability to distinguish between models, as will be shown in the following section. However, $\mathrm{S} / \sqrt{\mathrm{S}+\mathrm{B}}$ increases from the displayed values as $\sqrt{L / 10}$, improving the uncertainty by a factor of 3.2 for $100 \mathrm{fb}^{-1}$ and 5.5 for $300 \mathrm{fb}^{-1}$. 


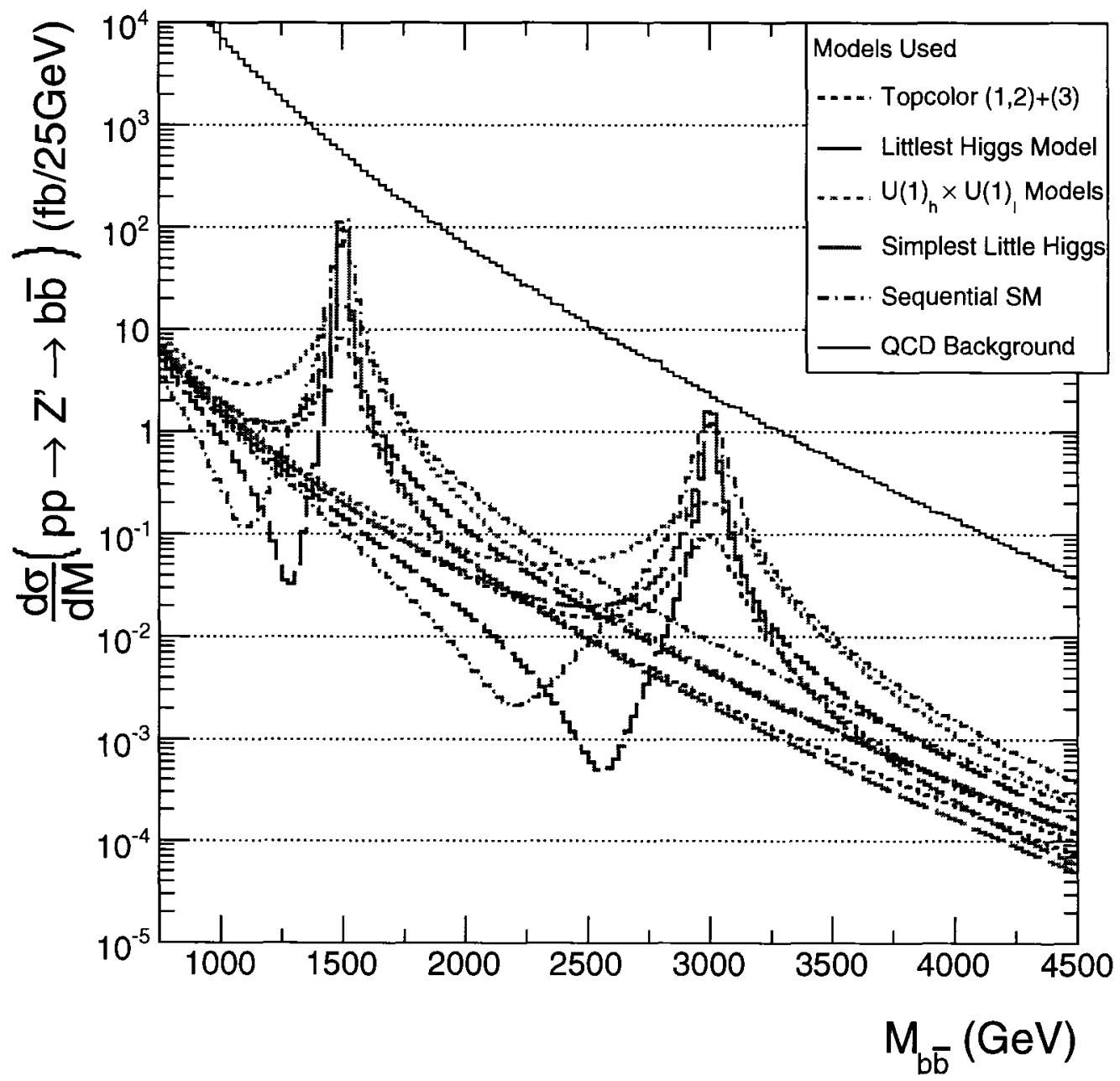

Figure 5.9: Invariant mass distribution for production of $b \bar{b}$ pairs with no cuts. QCD backgrounds are separated from the BSM EW processes $\left(M_{Z^{\prime}}=1500,3000 \mathrm{GeV}\right)$ to illustrate the relative strength of the signals. 


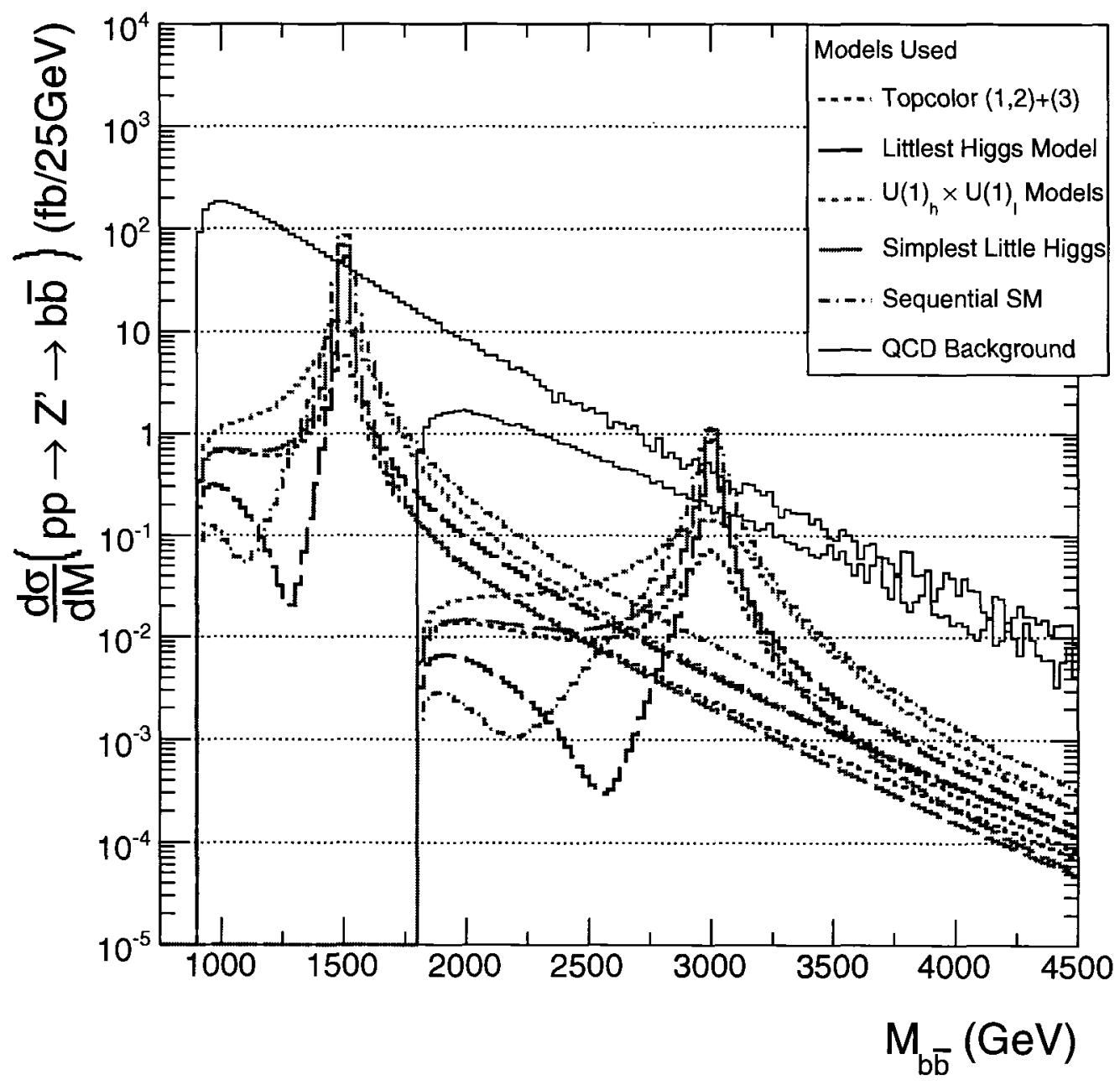

Figure 5.10: Invariant mass distribution for $b \bar{b}$ pair production with transverse momentum cut of $p_{T_{b}}>0.3 M_{Z^{\prime}}$. QCD backgrounds are separated from the BSM EW processes $\left(M_{Z^{\prime}}=1500,3000 \mathrm{GeV}\right)$ to illustrate the relative strength of the signals. The sharp vertical line arises due to the requirement of a minimum energy of $M_{t \bar{t}} \geq$ $2 p_{T}$ to pass the cut requirements. 
Table 5.2: Ratio of $\mathrm{S} / \sqrt{\mathrm{S}+\mathrm{B}}$ for $b \bar{b}$ and $t \bar{t}$ pairs for $10 \mathrm{fb}^{-1}$ integrated luminosity and optimistic efficiencies ( 0.5 and 0.1 respectively). Higher luminosity improves the ratio by a factor of $\sqrt{L / 10}$.

\begin{tabular}{|l|c|c|c|c|c|c|}
\hline & \multicolumn{2}{|c|}{$1000 \mathrm{GeV}$} & \multicolumn{2}{c|}{$2000 \mathrm{GeV}$} & \multicolumn{2}{|c|}{$3000 \mathrm{GeV}$} \\
\hline Model & $b \bar{b}$ & $t \bar{t}$ & $b \bar{b}$ & $t \bar{t}$ & $b \bar{b}$ & $t \bar{t}$ \\
\hline \hline E6 Model $-\chi$ & 25.90 & 1.96 & 6.73 & 0.66 & 2.18 & 0.23 \\
\hline E6 Model $-\eta$ & 17.38 & 1.36 & 4.65 & 0.47 & 1.54 & 0.16 \\
\hline E6 Model - $\psi$ & 13.35 & 8.82 & 3.79 & 2.90 & 1.37 & 1.04 \\
\hline LR Symmetric & 58.07 & 13.79 & 14.23 & 4.03 & 4.57 & 1.35 \\
\hline Alt. LR Symmetric & 4.44 & 11.11 & 1.36 & 3.49 & 0.52 & 1.25 \\
\hline HARV Model & 101.17 & 40.71 & 23.38 & 10.22 & 7.39 & 3.28 \\
\hline Sequential SM & 47.80 & 14.13 & 12.03 & 4.25 & 3.94 & 1.45 \\
\hline Topcolor $(1,2)+(3)$ & 4.72 & 4.71 & 1.41 & 1.43 & 0.52 & 0.52 \\
\hline Littlest Higgs Model & 26.10 & 10.25 & 7.06 & 3.07 & 2.41 & 1.07 \\
\hline Simplest Little Higgs & 34.49 & 15.62 & 9.16 & 4.34 & 3.10 & 1.47 \\
\hline Anomaly Free Simple LH & 32.99 & 13.80 & 8.76 & 3.87 & 2.96 & 1.32 \\
\hline 3-3-1 (2U 1D) & 29.68 & 8.57 & 7.85 & 2.72 & 2.64 & 0.95 \\
\hline 3-3-1 (1U 2D) & 34.49 & 15.62 & 9.16 & 4.34 & 3.10 & 1.47 \\
\hline$S U(2)_{h} \times S U(2)_{l}$ Models & 32.35 & 12.71 & 7.98 & 3.46 & 2.66 & 1.18 \\
\hline$U(1)_{h} \times U(1)_{l}$ Models & 11.35 & 14.37 & 3.20 & 4.00 & 1.17 & 1.39 \\
\hline
\end{tabular}


Table 5.3: Ratio of $\mathrm{S} / \sqrt{\mathrm{S}+\mathrm{B}}$ for $b \bar{b}$ and $t \bar{t}$ pairs for $10 \mathrm{fb}^{-1}$ integrated luminosity and pessimistic efficiencies ( 0.25 and 0.01 respectively). Higher luminosity improves the ratio by a factor of $\sqrt{L / 10}$.

\begin{tabular}{|l|c|c|c|c|c|c|}
\hline & \multicolumn{2}{|c|}{$1000 \mathrm{GeV}$} & \multicolumn{2}{|c|}{$2000 \mathrm{GeV}$} & \multicolumn{2}{|c|}{$3000 \mathrm{GeV}$} \\
\hline Model & $b \bar{b}$ & $t \bar{t}$ & $b \bar{b}$ & $t \bar{t}$ & $b \bar{b}$ & $t \bar{t}$ \\
\hline \hline E6 Model $-\chi$ & 18.31 & 1.96 & 6.73 & 0.21 & 1.54 & 0.07 \\
\hline E6 Model - $\eta$ & 12.29 & 1.36 & 4.65 & 0.15 & 1.09 & 0.05 \\
\hline E6 Model - $\psi$ & 9.44 & 8.82 & 3.79 & 0.92 & 0.97 & 0.33 \\
\hline LR Symmetric & 41.06 & 13.79 & 14.23 & 1.27 & 3.23 & 0.43 \\
\hline Alt. LR Symmetric & 3.14 & 11.11 & 1.36 & 1.10 & 0.37 & 0.40 \\
\hline HARV Model & 71.54 & 40.71 & 23.38 & 3.23 & 5.22 & 1.04 \\
\hline Sequential SM & 33.80 & 14.13 & 12.03 & 1.35 & 2.78 & 0.46 \\
\hline Topcolor $(1,2)+(3)$ & 3.34 & 4.71 & 1.41 & 0.45 & 0.37 & 0.17 \\
\hline Littlest Higgs Model & 18.46 & 10.25 & 7.06 & 0.97 & 1.71 & 0.34 \\
\hline Simplest Little Higgs & 24.39 & 15.62 & 9.16 & 1.37 & 2.19 & 0.46 \\
\hline Anomaly Free Simple LH & 23.33 & 13.80 & 8.76 & 1.23 & 2.09 & 0.42 \\
\hline 3-3-1 (2U 1D) & 20.98 & 8.57 & 7.85 & 0.86 & 1.87 & 0.30 \\
\hline 3-3-1 (1U 2D) & 24.39 & 15.62 & 9.16 & 1.37 & 2.19 & 0.46 \\
\hline$S U(2)_{h} \times S U(2)_{l}$ Models & 22.87 & 12.71 & 7.98 & 1.10 & 1.88 & 0.37 \\
\hline$U(1)_{h} \times U(1)_{l}$ Models & 8.03 & 14.37 & 3.20 & 1.26 & 0.83 & 0.44 \\
\hline
\end{tabular}




\subsubsection{Distinguishing between models using $R_{t / \mu}$ and $R_{b / \mu}$}

In comparing the quark to muon cross sections, the same cuts and windowing were applied to the muon channel as to the quark channel. The tighter invariant mass window results in a reduction in the expected event rate for leptons compared to that shown in Section 5.2. However, the lower efficiency for distinguishing top and bottom final states results in uncertainties being dominated by these measurements rather than the muon measurements.

In this analysis, I assume a perfect understanding of efficiencies, and apply them as ideal values without associated uncertainties. Though this is not accurate, it is used to simplify the analysis and show the viability of the measurement. Since the efficiencies used are estimates and real values are not currently known, this simplification should not affect the intent; with a low top efficiency, I expect that low statistics will contribute the most to the uncertainty for lower luminosities, rather than uncertainties in efficiencies.

Plotting $R_{b / \mu}$ vs $R_{t / \mu}$, the ratio of the bottom to muon cross sections and the ratio of the top to muon cross sections, respectively, acts as an excellent method for distinguishing between models. Figures 5.11 through 5.13 (pessimistic efficiencies) and 5.14 through 5.16 (optimistic efficiencies) show the effect of increasing luminosity on the region of uncertainty for both optimistic and pessimistic efficiencies with $M_{Z^{\prime}}=2000 \mathrm{GeV}$. The region is not meant to represent a contour in parameter space, but simply show the ability of this measurement to distinguish between models. 


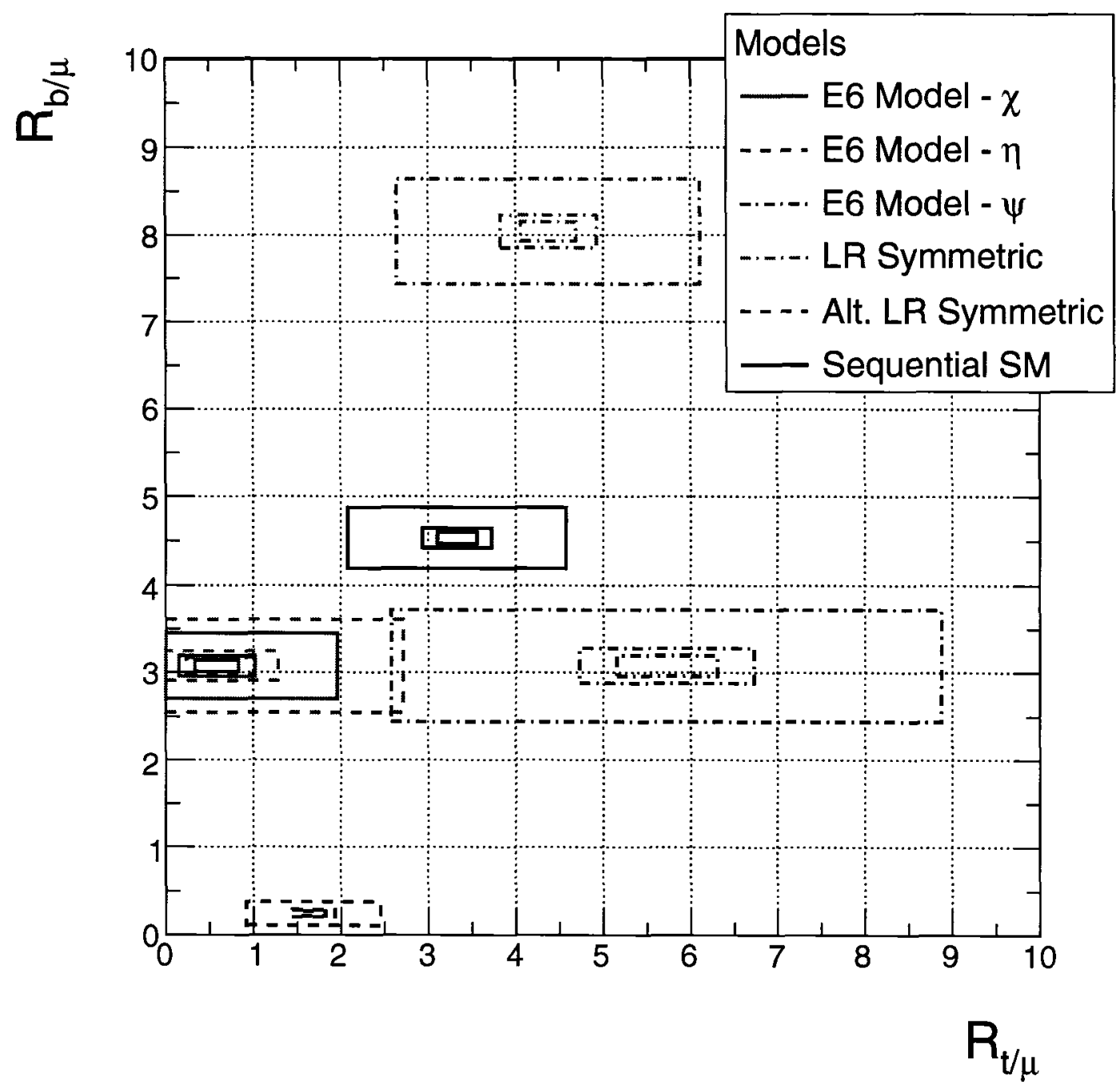

Figure 5.11: Plots of $1 \sigma$ uncertainty bounds for $R_{b / \mu}$ vs $R_{t / \mu}$ for $M_{Z^{\prime}}=2000 \mathrm{GeV}$ for the pessimistic tagging efficiency scenario: $\epsilon_{\mu}=0.96, \epsilon_{t}=0.01, \epsilon_{b}=0.25$. Concentric rectangles describe uncertainty region; tighter uncertainty limits correspond to increasing luminosity from $10 \mathrm{fb}^{-1}$ to $100 \mathrm{fb}^{-1}$ to $300 \mathrm{fb}^{-1}$. First set of models models split for clarity of plot. 


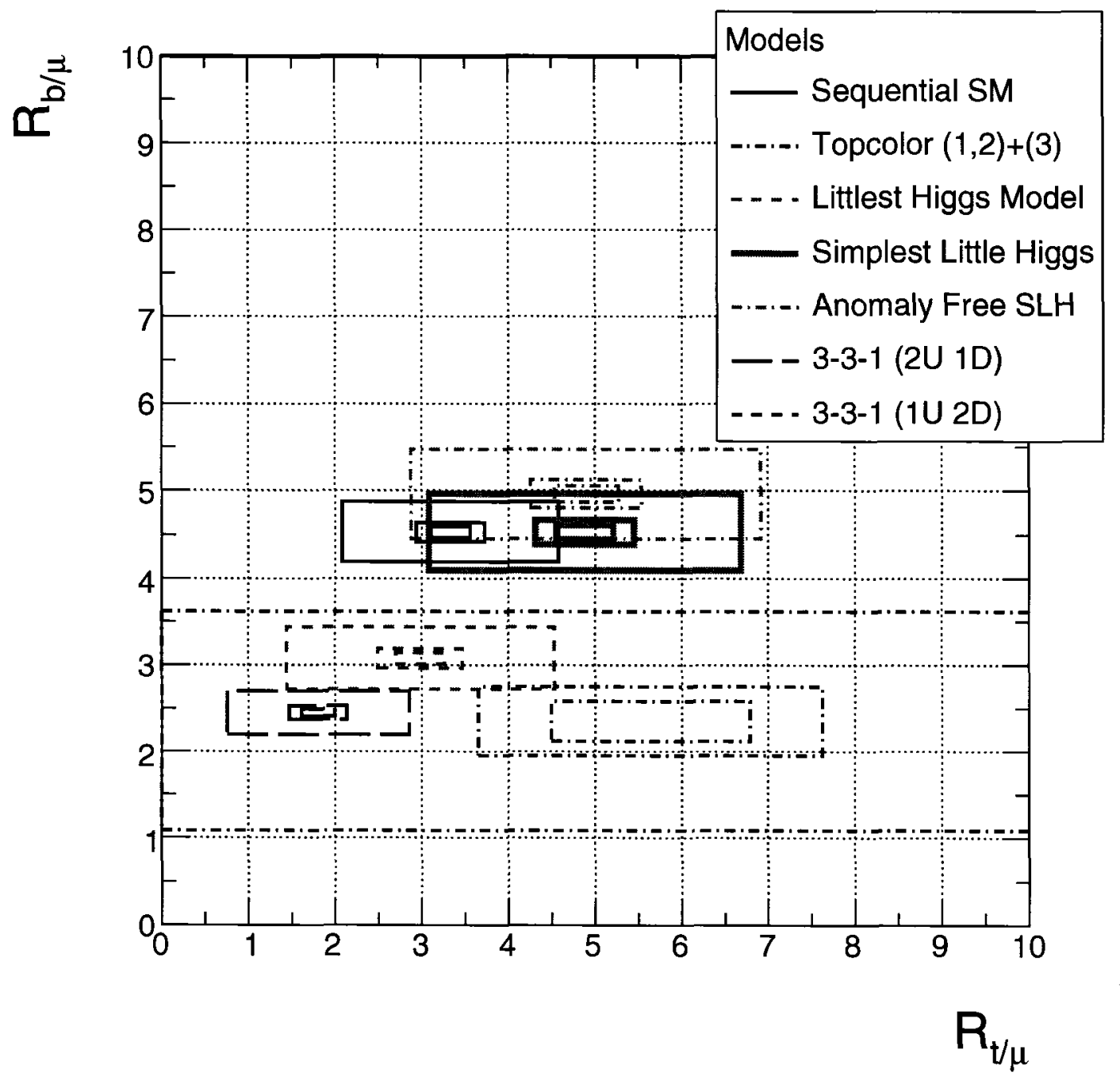

Figure 5.12: Plots of $1 \sigma$ uncertainty bounds for $R_{b / \mu}$ vs $R_{t / \mu}$ for $M_{Z^{\prime}}=2000 \mathrm{GeV}$ for the pessimistic tagging efficiency scenario: $\epsilon_{\mu}=0.96, \epsilon_{t}=0.01, \epsilon_{b}=0.25$. Concentric rectangles describe uncertainty region; tighter uncertainty limits correspond to increasing luminosity from $10 \mathrm{fb}^{-1}$ to $100 \mathrm{fb}^{-1}$ to $300 \mathrm{fb}^{-1}$. Second set of models - models split for clarity of plot. 


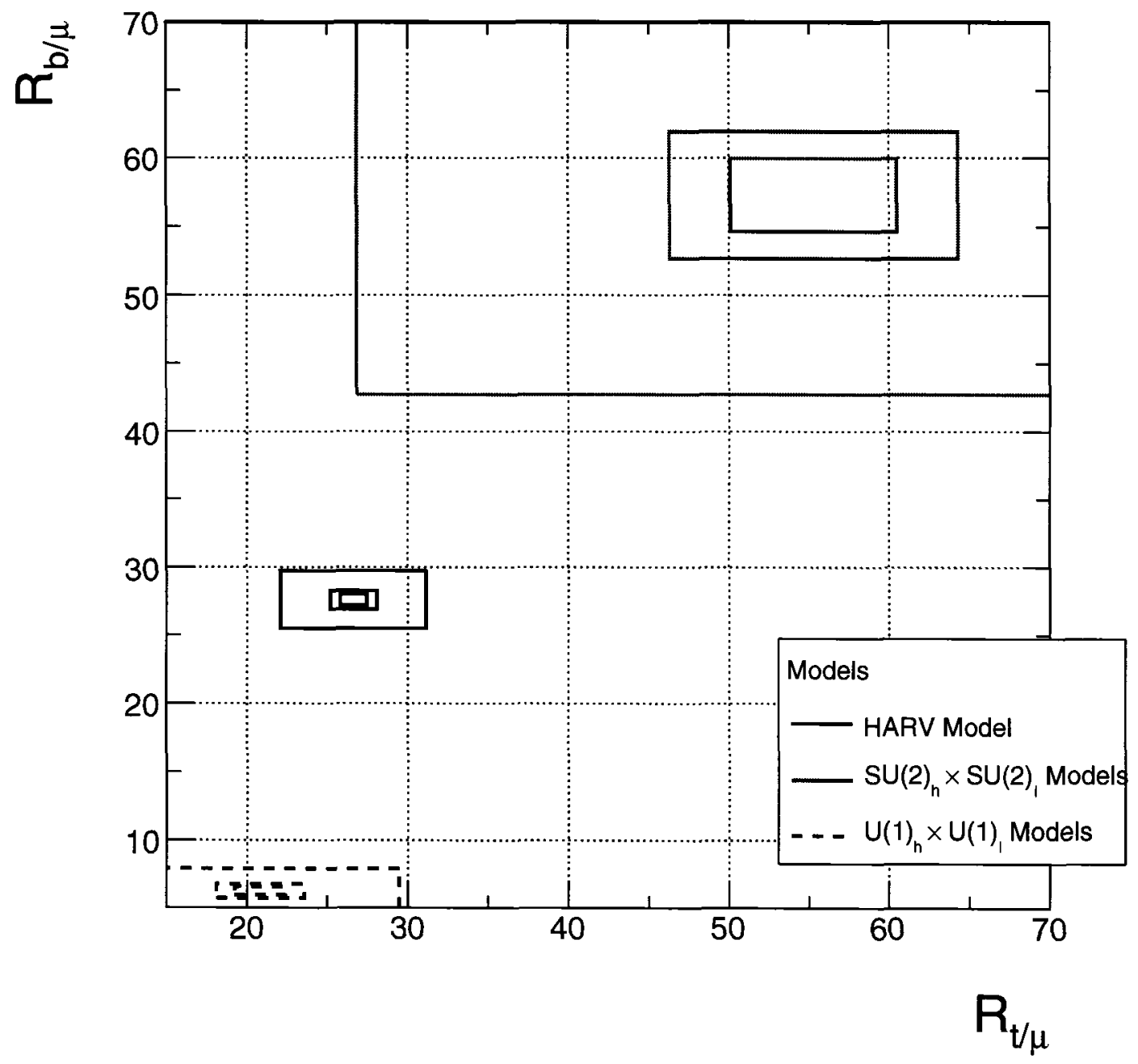

Figure 5.13: Plots of $1 \sigma$ uncertainty bounds for $R_{b / \mu}$ vs $R_{t / \mu}$ for $M_{Z^{\prime}}=2000 \mathrm{GeV}$ for the pessimistic tagging efficiency scenario: $\epsilon_{\mu}=0.96, \epsilon_{t}=0.01, \epsilon_{b}=0.25$. Concentric rectangles describe uncertainty region; tighter uncertainty limits correspond to increasing luminosity from $10 \mathrm{fb}^{-1}$ to $100 \mathrm{fb}^{-1}$ to $300 \mathrm{fb}^{-1}$. Models include those with distinctive signatures. 


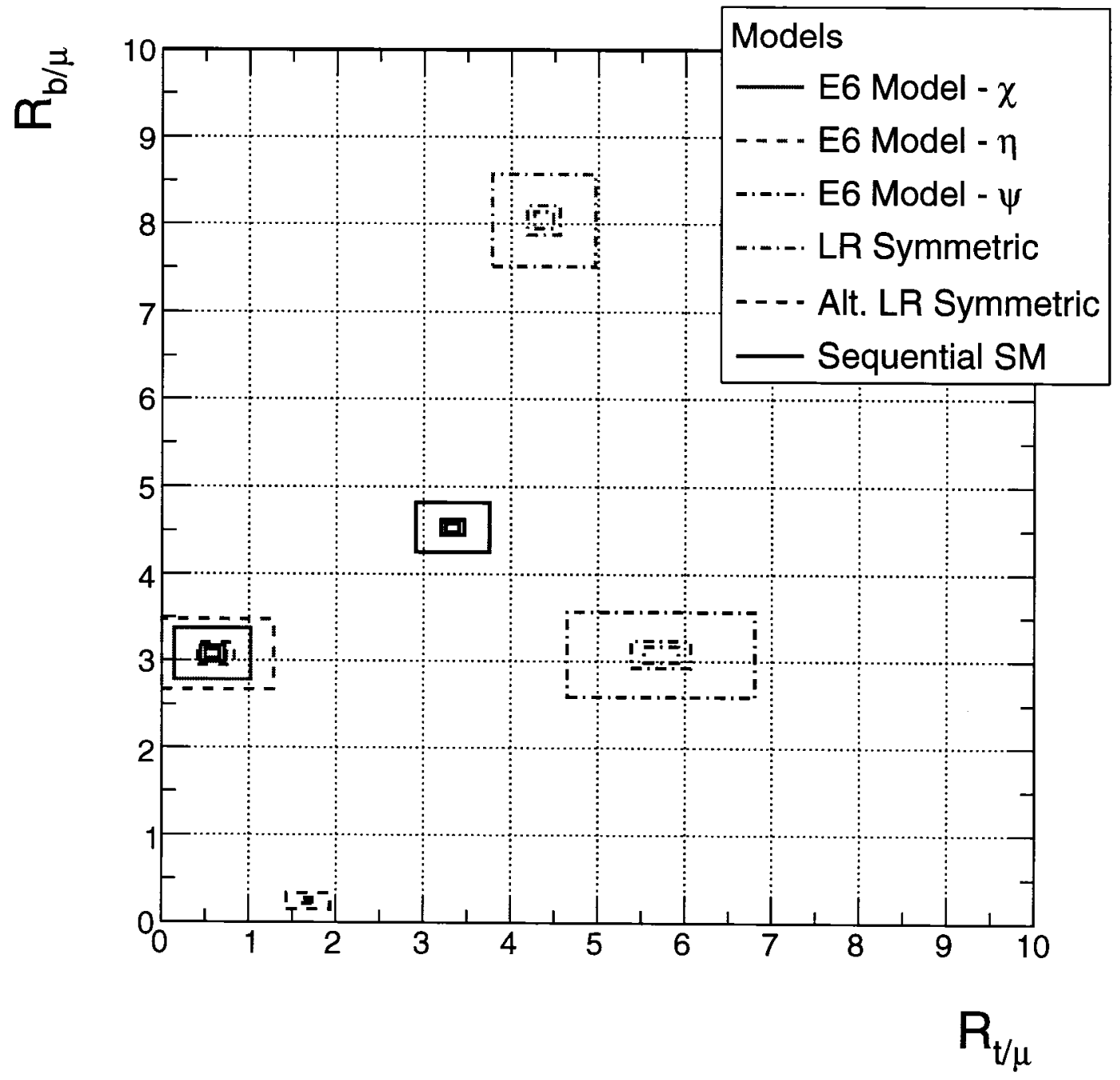

Figure 5.14: Plots of $1 \sigma$ uncertainty bounds for $R_{b / \mu}$ vs $R_{t / \mu}$ for $M_{Z^{\prime}}=2000 \mathrm{GeV}$ for the optimistic tagging efficiency scenario: $\epsilon_{\mu}=0.98, \epsilon_{t}=0.10, \epsilon_{b}=0.50$. Concentric rectangles describe uncertainty region; tighter uncertainty limits correspond to increasing luminosity from $10 \mathrm{fb}^{-1}$ to $100 \mathrm{fb}^{-1}$ to $300 \mathrm{fb}^{-1}$. First set of models models split for clarity of plot. 


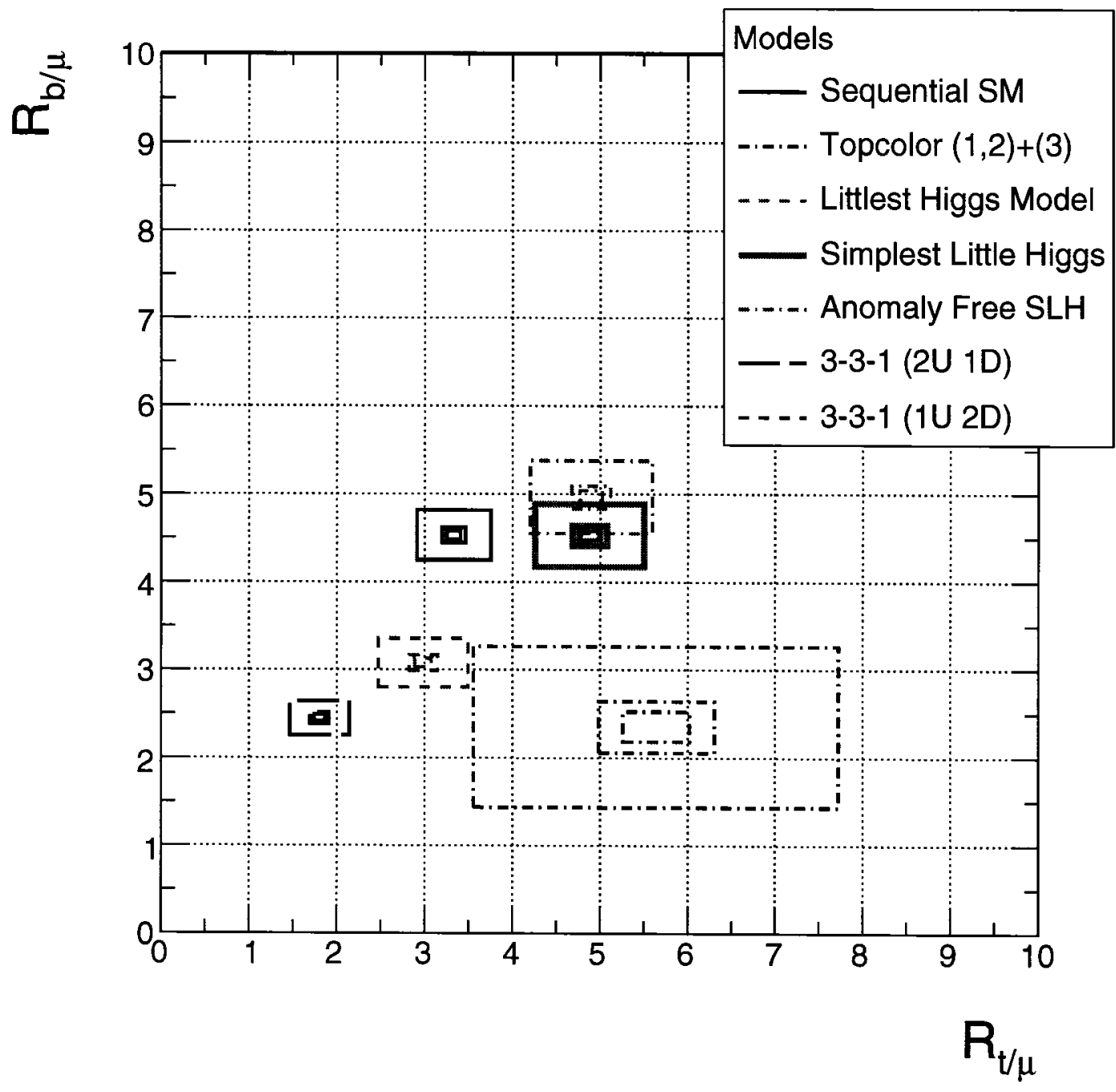

Figure 5.15: Plots of $1 \sigma$ uncertainty bounds for $R_{b / \mu}$ vs $R_{t / \mu}$ for $M_{Z^{\prime}}=2000 \mathrm{GeV}$ for the optimistic tagging efficiency scenario: $\epsilon_{\mu}=0.98, \epsilon_{t}=0.10, \epsilon_{b}=0.50$. Concentric rectangles describe uncertainty region; tighter uncertainty limits correspond to increasing luminosity from $10 \mathrm{fb}^{-1}$ to $100 \mathrm{fb}^{-1}$ to $300 \mathrm{fb}^{-1}$. Second set of models - models split for clarity of plot. 


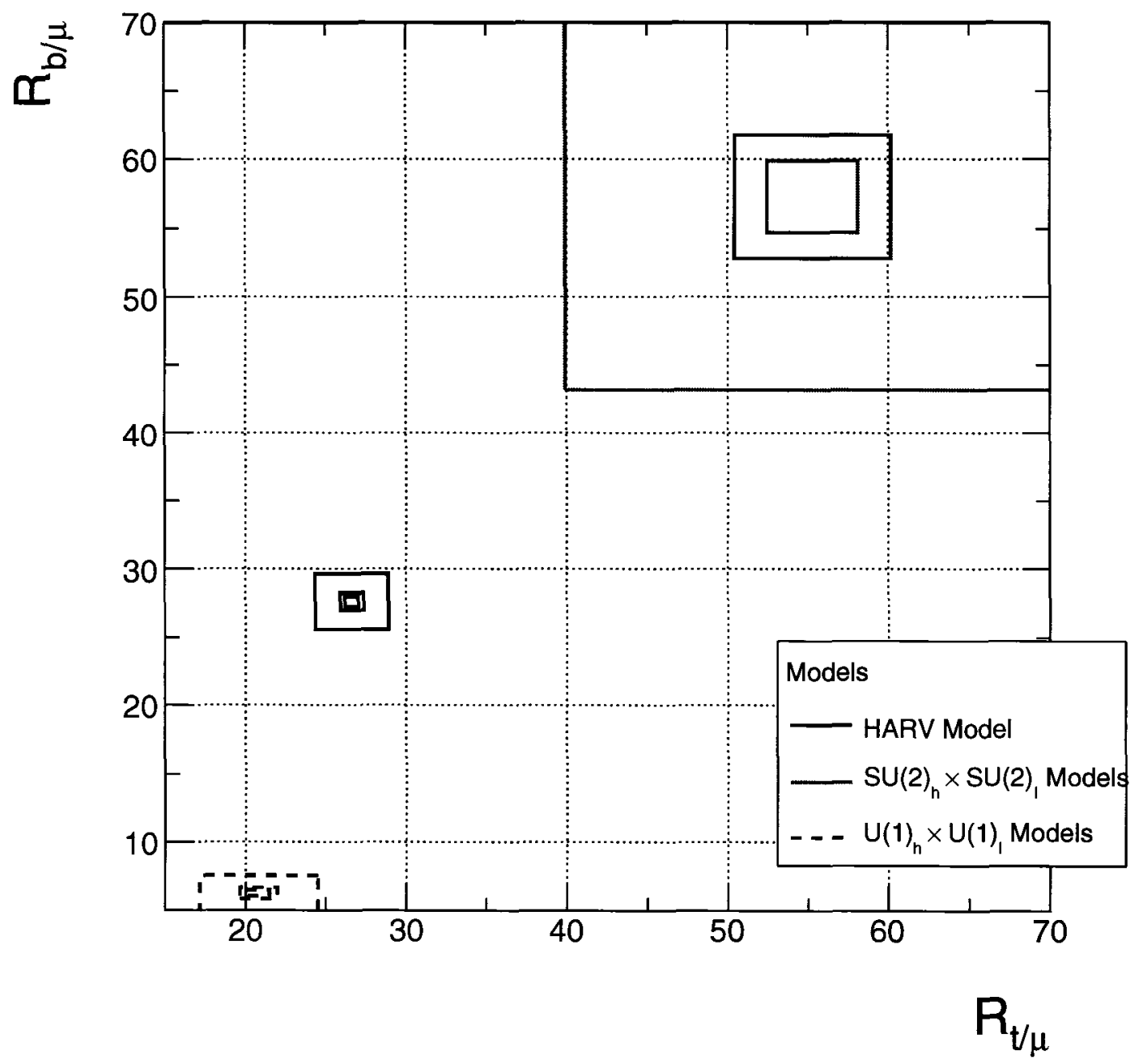

Figure 5.16: Plots of $1 \sigma$ uncertainty bounds for $R_{b / \mu}$ vs $R_{t / \mu}$ for $M_{Z^{\prime}}=2000 \mathrm{GeV}$ for the optimistic tagging efficiency scenario: $\epsilon_{\mu}=0.98, \epsilon_{t}=0.10, \epsilon_{b}=0.50$. Concentric rectangles describe uncertainty region; tighter uncertainty limits correspond to increasing luminosity from $10 \mathrm{fb}^{-1}$ to $100 \mathrm{fb}^{-1}$ to $300 \mathrm{fb}^{-1}$. Models include those with distinctive signatures.

Figures 5.17 through 5.19 (pessimistic efficiencies) and 5.20 through 5.22 (optimistic efficiencies) show a similar analysis, except that an integrated luminosity of $100 \mathrm{fb}^{-1}$ was chosen, and increasing regions of interest are due to an increase of $M_{Z^{\prime}}$. The smallest region results from considering a $1000 \mathrm{GeV} Z^{\prime}$, where effects from the mass terms affect the top ratio measurement significantly. At $1500 \mathrm{GeV}$, the centroid 
of the region of interest in most cases is significantly closer to that of the higher masses $(2000,2500$ and $3000 \mathrm{GeV})$. From this analysis, it becomes clear that a large $Z^{\prime}$ mass results in significant difficulties for distinguishing between models.

Of interest is the difference between the Anomaly Free Simple Little Higgs model and the 3-3-1 (1U 2D) (and Simplest Little Higgs) model. At optimistic efficiencies, the models are only distinguishable for masses below $2500 \mathrm{GeV}$. For pessimistic efficiencies, these models are only distinguishable from each other, and from the SSM, for masses below approximately $2000 \mathrm{GeV}$.

In general, most models can be distinguished from each other at low luminosities, with high quark tagging efficiencies. Should the efficiencies turn out to be closer to the pessimistic range, many models can still be distinguished, though greater statistics will be required to distinguish between some models. The most distinct, and easily distinguished, models in each case are the Left Right Symmetric and Alternate Left Right Symmetric models, the Topcolor model, the HARV model, models with a $U(1)_{h} \times U(1)_{l}$, and the $S U(2)_{h} \times S U(2)_{l}$ models.

It should be noted that several models will be indistinguishable regardless of statistics and efficiencies. Specifically, the Simplest Little Higgs model and the 3-3-1 Model (1U 2D) result in identical ratios and signal strengths. These models have identical couplings. Additionally, the E6 models $\chi$ and $\eta$, and the E6 $\psi$ and Topcolor models, result in almost identical ratios. For the $\chi$ and $\eta$ models the $b$ sector ratios are identical, with a $6 \%$ difference in the $t$ sector ratios. The Topcolor model and $\psi$ model are difficult to distinguish, with a $25 \%$ difference in the $b / \mu$ ratio measurement, and an $8 \%$ difference in the $t / \mu$ ratio measurement, as the large uncertainty in the Topcolor model overlaps significantly. 


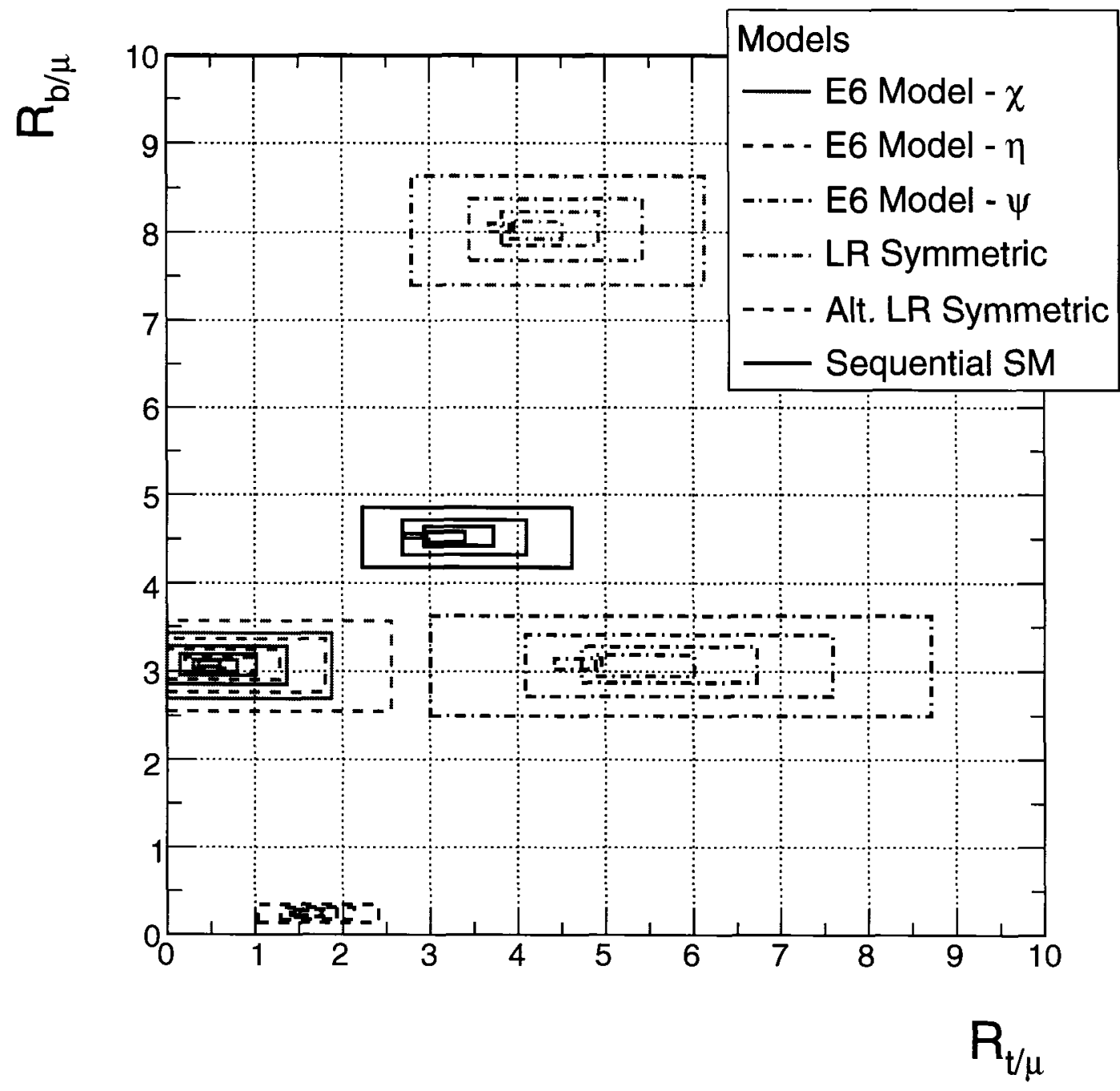

Figure 5.17: Plots of $1 \sigma$ uncertainty bounds for $R_{b / \mu}$ vs $R_{t / \mu}$ for integrated luminosity, $\mathrm{L}=100 \mathrm{fb}^{-1}$. Efficiencies for final states are from the pessimistic scenario: $\epsilon_{\mu}=0.96$, $\epsilon_{t}=0.01, \epsilon_{b}=0.25$. Regions of interest increase in size with increasing $M_{Z^{\prime}}(1000$, $1500,2000,2500,3000 \mathrm{GeV}$ ). The centroid of the regions shift significantly between $M_{Z^{\prime}}=1000 \mathrm{GeV}, 1500 \mathrm{GeV}$ and $>1500 \mathrm{GeV}$ due to the decreasing effect of the mass term in $t \bar{t}$ cross section. First set of models - models split for clarity of plot. 


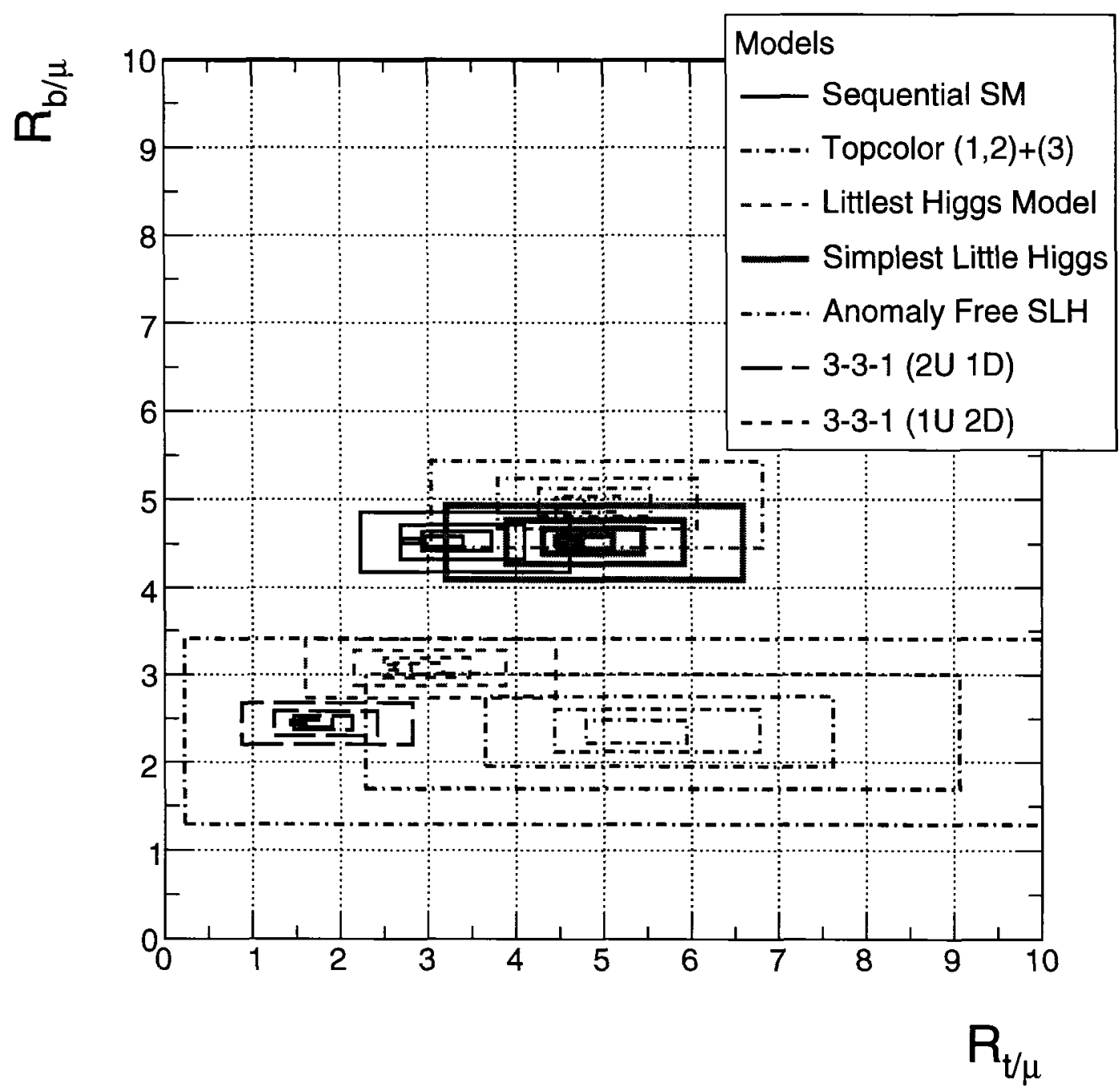

Figure 5.18: Plots of $1 \sigma$ uncertainty bounds for $R_{b / \mu}$ vs $R_{t / \mu}$ for integrated luminosity, $\mathrm{L}=100 \mathrm{fb}^{-1}$. Efficiencies for final states are from the pessimistic scenario: $\epsilon_{\mu}=0.96$, $\epsilon_{t}=0.01, \epsilon_{b}=0.25$. Regions of interest increase in size with increasing $M_{Z^{\prime}}$ (1000, $1500,2000,2500,3000 \mathrm{GeV})$. The centroid of the regions shift significantly between $M_{Z^{\prime}}=1000 \mathrm{GeV}, 1500 \mathrm{GeV}$ and $>1500 \mathrm{GeV}$ due to the decreasing effect of the mass term in $t \bar{t}$ cross section. Second set of models - models split for clarity of plot. 


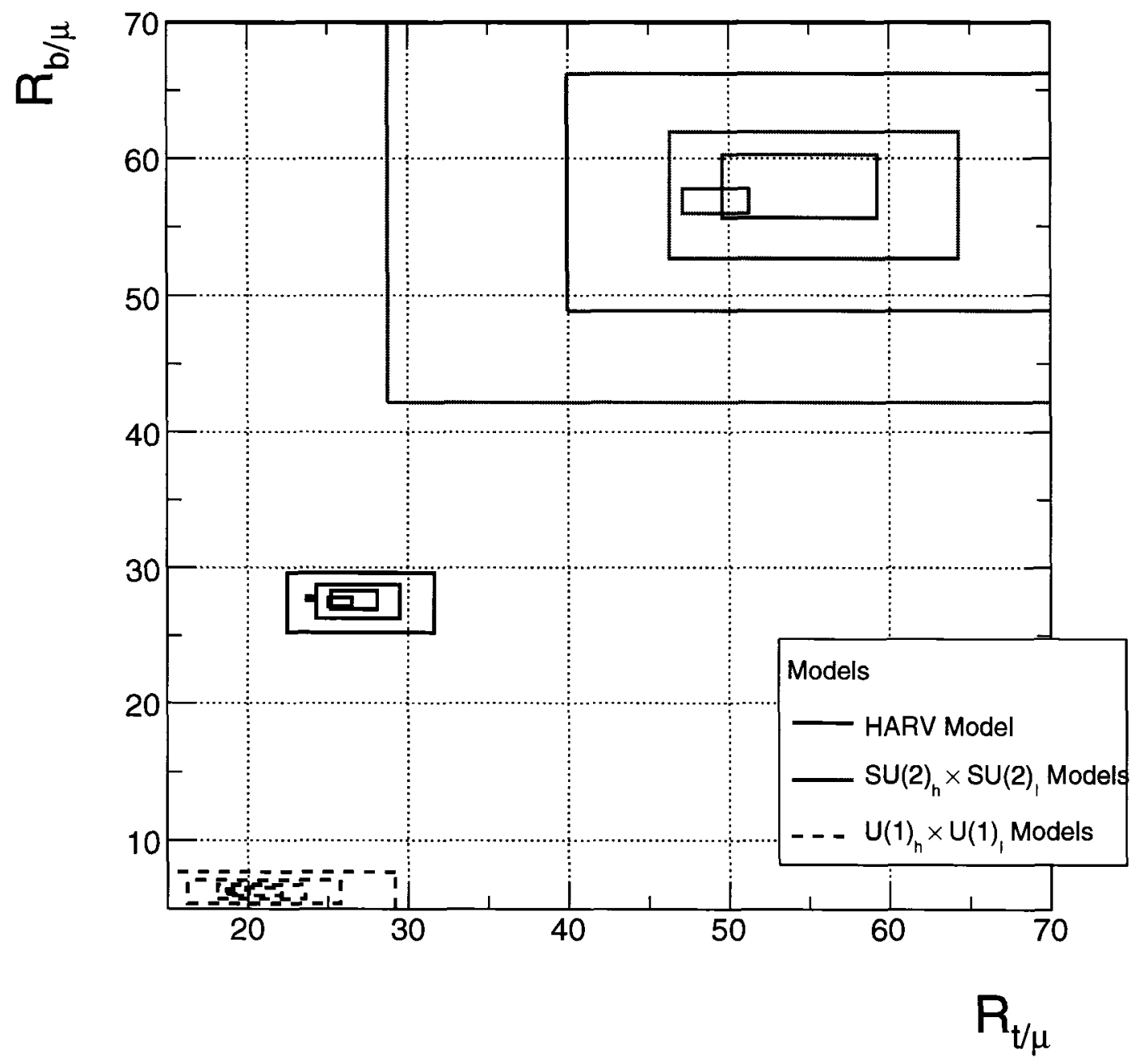

Figure 5.19: Plots of $1 \sigma$ uncertainty bounds for $R_{b / \mu}$ vs $R_{t / \mu}$ for integrated luminosity, $\mathrm{L}=100 \mathrm{fb}^{-1}$. Efficiencies for final states are from the pessimistic scenario: $\epsilon_{\mu}=0.96$, $\epsilon_{t}=0.01, \epsilon_{b}=0.25$. Regions of interest increase in size with increasing $M_{Z^{\prime}}$ (1000, $1500,2000,2500,3000 \mathrm{GeV})$. The centroid of the regions shift significantly between $M_{Z^{\prime}}=1000 \mathrm{GeV}, 1500 \mathrm{GeV}$ and $>1500 \mathrm{GeV}$ due to the decreasing effect of the mass term in $t \bar{t}$ cross section. Models include those with distinctive signatures. 


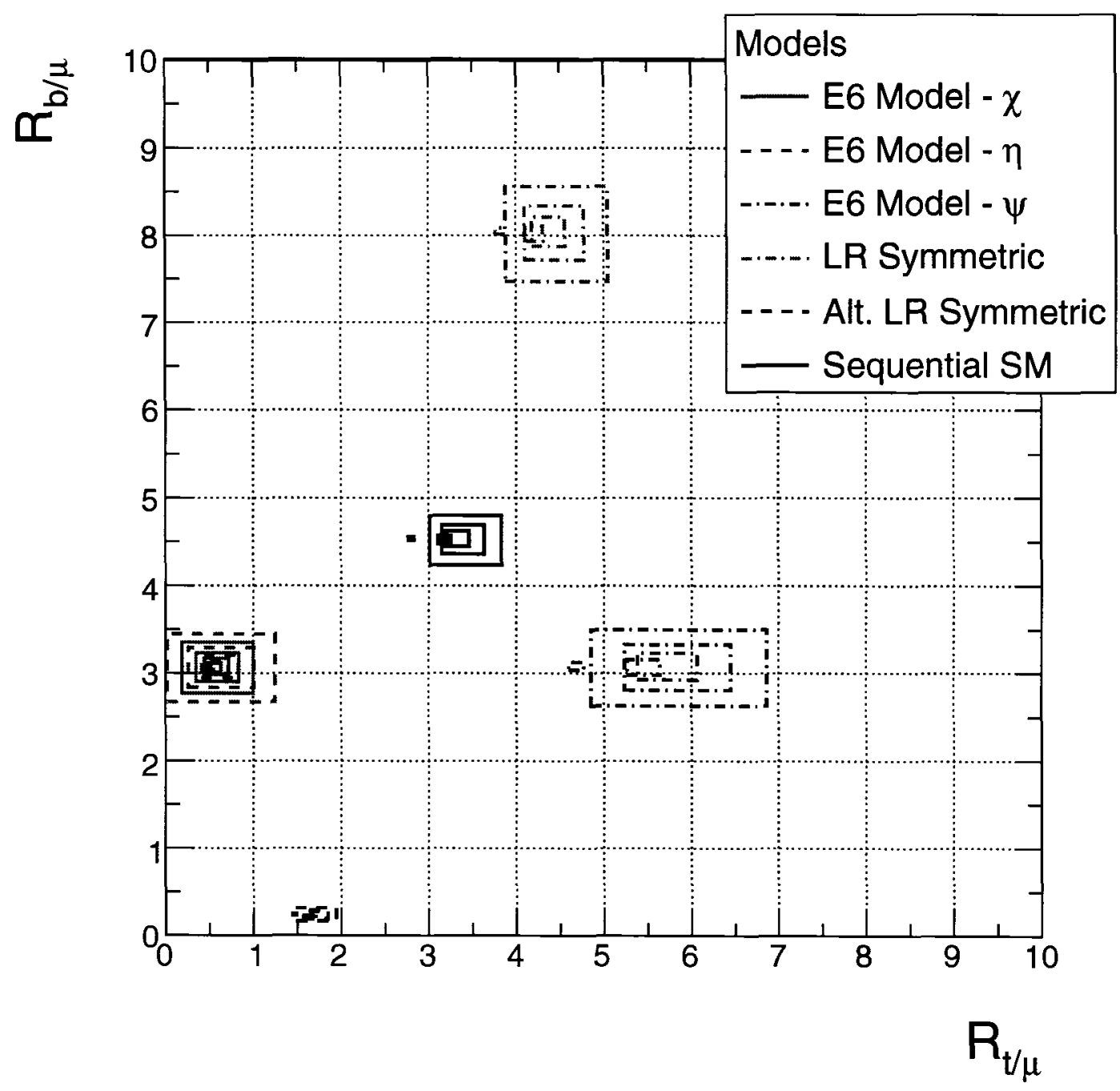

Figure 5.20: Plots of $1 \sigma$ uncertainty bounds for $R_{b / \mu}$ vs $R_{t / \mu}$ for integrated luminosity, $\mathrm{L}=100 \mathrm{fb}^{-1}$. Efficiencies for final states are from the optimistic scenario: $\epsilon_{\mu}=0.98$, $\epsilon_{t}=0.10, \epsilon_{b}=0.50$. Regions of interest increase in size with increasing $M_{Z^{\prime}}(1000$, $1500,2000,2500,3000 \mathrm{GeV}$ ). The centroid of the regions shift significantly between $M_{Z^{\prime}}=1000 \mathrm{GeV}, 1500 \mathrm{GeV}$ and $>1500 \mathrm{GeV}$ due to the decreasing effect of the mass term in $t \bar{t}$ cross section. First set of models - models split for clarity of plot. 


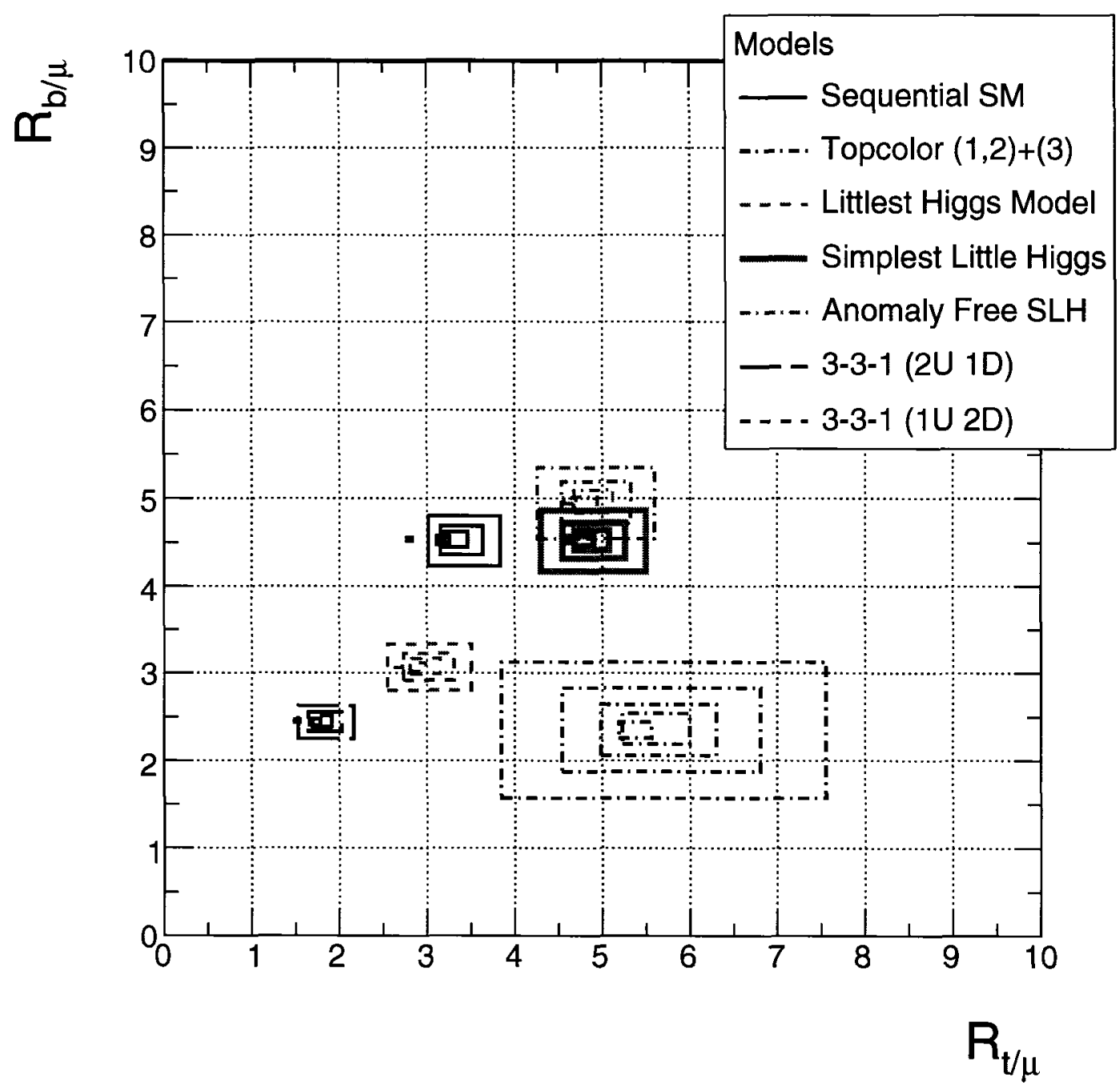

Figure 5.21: Plots of $1 \sigma$ uncertainty bounds for $R_{b / \mu}$ vs $R_{t / \mu}$ for integrated luminosity, $\mathrm{L}=100 \mathrm{fb}^{-1}$. Efficiencies for final states are from the pessimistic scenario: $\epsilon_{\mu}=0.98$, $\epsilon_{t}=0.10, \epsilon_{b}=0.50$. Regions of interest increase in size with increasing $M_{Z^{\prime}}(1000$, $1500,2000,2500,3000 \mathrm{GeV}$ ). The centroid of the regions shift significantly between $M_{Z^{\prime}}=1000 \mathrm{GeV}, 1500 \mathrm{GeV}$ and $>1500 \mathrm{GeV}$ due to the decreasing effect of the mass term in $t \bar{t}$ cross section. Second set of models - models split for clarity of plot. 


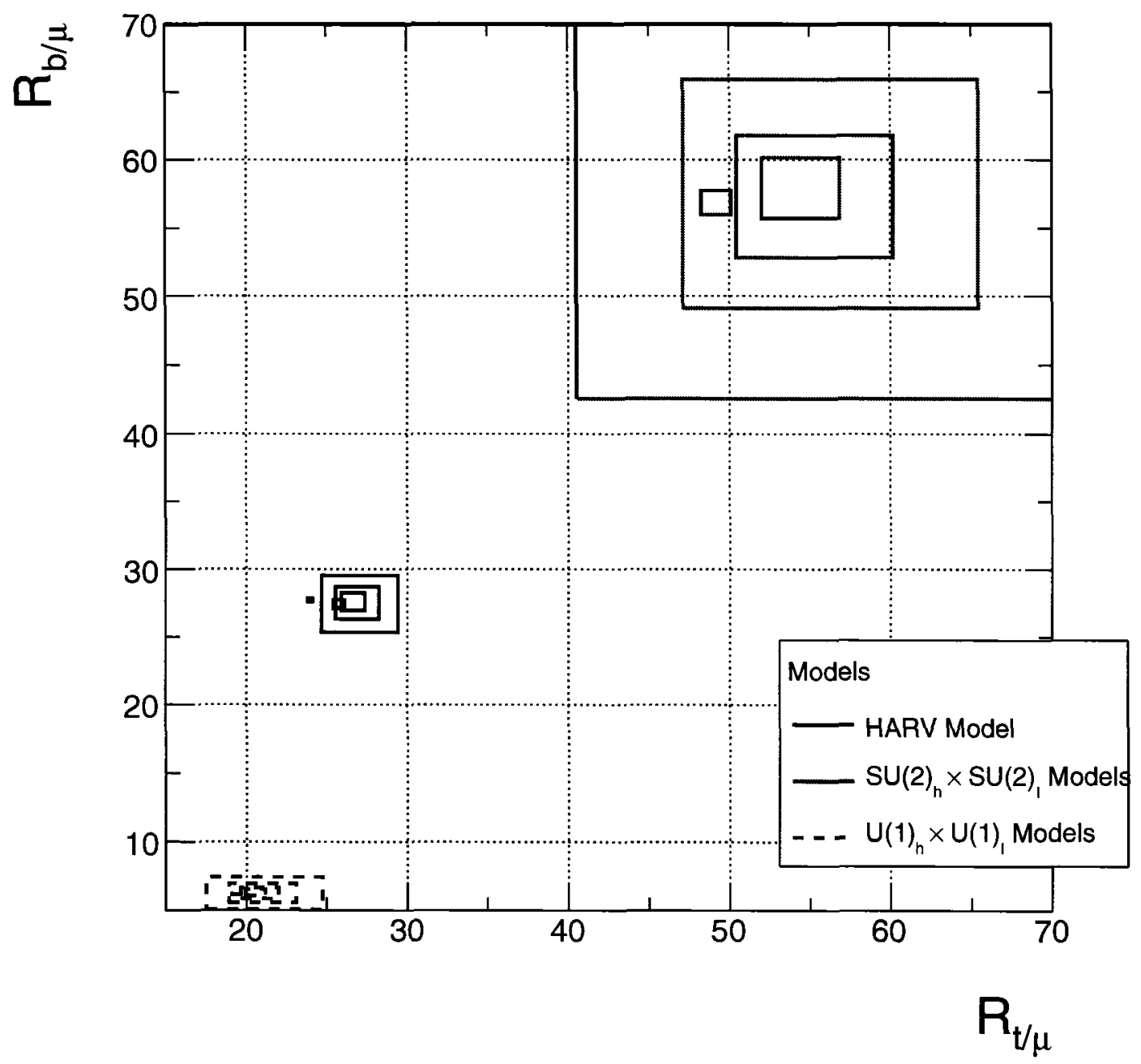

Figure 5.22: Plots of $1 \sigma$ uncertainty bounds for $R_{b / \mu}$ vs $R_{t / \mu}$ for integrated luminosity, $\mathrm{L}=100 \mathrm{fb}^{-1}$. Efficiencies for final states are from the optimistic scenario: $\epsilon_{\mu}=0.98$, $\epsilon_{t}=0.10, \epsilon_{b}=0.50$. Regions of interest increase in size with increasing $M_{Z^{\prime}}(1000$, $1500,2000,2500,3000 \mathrm{GeV}$ ). The centroid of the regions shift significantly between $M_{Z^{\prime}}=1000 \mathrm{GeV}, 1500 \mathrm{GeV}$ and $>1500 \mathrm{GeV}$ due to the decreasing effect of the mass term in $t \bar{t}$ cross section. Models include those with distinctive signatures. 
In some cases, the measurement of these ratios may be indicators of nongeneration universality, as is evident in the measurements of the $U(1)_{h} \times U(1)_{l}$ and $S U(2)_{h} \times S U(2)_{l}$ models. However, the HARV model provides a counter example of how a measurement of large values can occur from a model with generation universality. Depending on the mixing parameters involved in these three models, they may become completely indistinguishable. Due to this, a measurement to determine the nature of the couplings to the generations, as described in Section 5.4, would provide a complementary analysis.

The leptophobic nature of the HARV model illustrates a complication with this measurement technique in general - models that are completely leptophobic (such as certain versions of the Topcolor model) or simply highly leptophobic may result in a low event rate for muons. In this case, the mixing parameter between the $S U(2)_{l} \times S U(2)_{q}$ in the HARV model resulted in a large enough event rate in the muon channel.

\subsection{Determining Generation Universality in $Z^{\prime}$}

\subsubsection{Lepton channel}

In general, a model independent study of generation universality will include measurements of the ratio of all three possible combinations of $\tau, \mu$ and $e$ cross sections. As mentioned, Topcolor models specifically present a scenario where the coupling preference of the $Z^{\prime}$ can include combinations other than a simple preference for the third generation. However, all the models considered here have either generation universal couplings or a preferential coupling to only third generation fermions. 
In this case, the most precise measurement will be the ratio of $\tau$ to $\mu$ event rates, as the muon efficiency is higher than that of electrons. In the analysis that follows, I assume that the ability to distinguish between jets arising from the hadronic decay of a $\tau$ lepton and from the QCD background is included in the efficiency and fake rates.

Tables 5.4 and 5.5 show the expected ratio $R_{\tau / \mu}$ for each model with optimistic and pessimistic efficiencies, respectively, with $10 \mathrm{fb}^{-1}$ integrated luminosity. Uncertainties decrease by a factor of $\sqrt{10 / L}$ for higher luminosities.

The calculations of the cross sections involved here use the same statistics, which results in similar deviations from the ideal values. However, the expected uncertainty in the value of the ratio is larger than these statistical fluctuations in each case. A tighter invariant mass window and higher statistics in the Monte-Carlo would resolve these issues.

Figures 5.23 through 5.30 are graphical representations of the data presented in Tables 5.4 and 5.5, under different scenarios of detection efficiencies and luminosity. The first two figures present the case of pessimistic efficiency for $10 \mathrm{fb}^{-1}$ integrated luminosity, followed by two in the optimistic efficiency. In Figures 5.23, the uncertainty bounds for a $\geq 2000 \mathrm{GeV} Z^{\prime}$ are outside the scale of the axes for most models, and the same for Figure 5.25 for $M_{Z^{\prime}} \geq 3000 \mathrm{GeV}$. From this, it is apparent that the low statistics in these cases result in the indistinguishability of generation universality from a small preference for third generation fermions. Figures 5.24 and 5.26 expand the axis to show a larger region. From these, the results for models with preferential couplings are apparent. It is clear that the coupling strength difference is only distinct for $M_{Z^{\prime}} \approx 1000 \mathrm{GeV}$. Large uncertainties occur for a higher mass $Z^{\prime}$ due to the significantly suppressed $\mu$ final state, which results in very few $\mu$ pair events observed. 
Table 5.4: $\quad R_{\tau / \mu}$ for a 1,2 and $3 \mathrm{TeV} Z^{\prime}$. The uncertainty listed corresponds to an integrated luminosity of $\mathrm{L}=10 \mathrm{fb}^{-1}$ and optimistic $\tau$ efficiency of 0.2 .

\begin{tabular}{|l|c|c|c|c|}
\hline Model & Ideal & $1000 \mathrm{GeV}$ & $2000 \mathrm{GeV}$ & $3000 \mathrm{GeV}$ \\
\hline \hline E6 Model $-\chi$ & 1.000 & $0.997 \pm 0.062$ & $1.000 \pm 0.315$ & $1.005 \pm 1.087$ \\
\hline E6 Model $-\eta$ & 1.000 & $0.996 \pm 0.079$ & $1.000 \pm 0.399$ & $1.006 \pm 1.377$ \\
\hline E6 Model $-\psi$ & 1.000 & $0.998 \pm 0.092$ & $1.001 \pm 0.452$ & $1.006 \pm 1.486$ \\
\hline LR Symmetric & 1.000 & $0.998 \pm 0.061$ & $1.000 \pm 0.306$ & $1.003 \pm 1.031$ \\
\hline Alt. LR Symmetric & 1.000 & $0.999 \pm 0.043$ & $1.000 \pm 0.210$ & $1.004 \pm 0.679$ \\
\hline HARV Model & 1.000 & $0.999 \pm 0.069$ & $1.001 \pm 0.339$ & $1.001 \pm 1.117$ \\
\hline Sequential SM & 1.000 & $0.999 \pm 0.050$ & $1.001 \pm 0.251$ & $1.002 \pm 0.838$ \\
\hline Topcolor $(1,2)+(3)$ & 6.809 & $6.970 \pm 0.458$ & $7.064 \pm 2.278$ & $7.162 \pm 7.563$ \\
\hline Littlest Higgs Model & 1.000 & $0.998 \pm 0.062$ & $1.000 \pm 0.306$ & $1.004 \pm 1.020$ \\
\hline Simplest Little Higgs & 1.000 & $0.998 \pm 0.064$ & $1.000 \pm 0.313$ & $1.004 \pm 1.037$ \\
\hline Anomaly Free Simple LH & 1.000 & $0.998 \pm 0.069$ & $1.000 \pm 0.340$ & $1.004 \pm 1.132$ \\
\hline 3-3-1 $(2 \mathrm{U} 1 \mathrm{D})$ & 1.000 & $0.998 \pm 0.051$ & $1.000 \pm 0.253$ & $1.004 \pm 0.848$ \\
\hline 3-3-1 $(1 \mathrm{U}$ 2D) & 1.000 & $0.998 \pm 0.064$ & $1.000 \pm 0.313$ & $1.004 \pm 1.037$ \\
\hline$S U(2)_{h} \times S U(2)_{l}$ Models & 18.184 & $19.104 \pm 2.166$ & $19.689 \pm 11.805$ & $20.293 \pm 41.541$ \\
\hline$U(1)_{h} \times U(1)_{l}$ Models & 18.178 & $18.496 \pm 1.015$ & $18.695 \pm 5.105$ & $18.875 \pm 16.826$ \\
\hline
\end{tabular}


Table 5.5: $\quad R_{\tau / \mu}$ for a 1,2 and $3 \mathrm{TeV} Z^{\prime}$. The uncertainty listed corresponds to an integrated luminosity of $\mathrm{L}=10 \mathrm{fb}^{-1}$ and pessimistic $\tau$ efficiency of 0.01 .

\begin{tabular}{|l|c|c|c|c|}
\hline Model & Ideal & $1000 \mathrm{GeV}$ & $2000 \mathrm{GeV}$ & $3000 \mathrm{GeV}$ \\
\hline \hline E6 Model $-\chi$ & 1.000 & $0.997 \pm 0.253$ & $1.000 \pm 1.283$ & $1.005 \pm 4.428$ \\
\hline E6 Model $-\eta$ & 1.000 & $0.996 \pm 0.320$ & $1.000 \pm 1.626$ & $1.006 \pm 5.611$ \\
\hline E6 Model $-\psi$ & 1.000 & $0.998 \pm 0.374$ & $1.001 \pm 1.844$ & $1.006 \pm 6.054$ \\
\hline LR Symmetric & 1.000 & $0.998 \pm 0.250$ & $1.000 \pm 1.248$ & $1.003 \pm 4.202$ \\
\hline Alt. LR Symmetric & 1.000 & $0.999 \pm 0.177$ & $1.000 \pm 0.855$ & $1.004 \pm 2.767$ \\
\hline HARV Model & 1.000 & $0.999 \pm 0.280$ & $1.001 \pm 1.381$ & $1.001 \pm 4.552$ \\
\hline Sequential SM & 1.000 & $0.999 \pm 0.206$ & $1.001 \pm 1.023$ & $1.002 \pm 3.416$ \\
\hline Topcolor $(1,2)+(3)$ & 6.809 & $6.970 \pm 1.309$ & $7.064 \pm 6.485$ & $7.162 \pm 21.432$ \\
\hline Littlest Higgs Model & 1.000 & $0.998 \pm 0.252$ & $1.000 \pm 1.248$ & $1.004 \pm 4.155$ \\
\hline Simplest Little Higgs & 1.000 & $0.998 \pm 0.260$ & $1.000 \pm 1.276$ & $1.004 \pm 4.224$ \\
\hline Anomaly Free Simple LH & 1.000 & $0.998 \pm 0.281$ & $1.000 \pm 1.387$ & $1.004 \pm 4.612$ \\
\hline $3-3-1(2 \mathrm{U} 1 \mathrm{D})$ & 1.000 & $0.998 \pm 0.208$ & $1.000 \pm 1.033$ & $1.004 \pm 3.457$ \\
\hline $3-3-1(1 \mathrm{U}$ 2D) & 1.000 & $0.998 \pm 0.260$ & $1.000 \pm 1.276$ & $1.004 \pm 4.224$ \\
\hline$S U(2)_{h} \times S U(2)_{l}$ Models & 18.184 & $19.104 \pm 4.288$ & $19.689 \pm 22.874$ & $20.293 \pm 79.129$ \\
\hline$U(1)_{h} \times U(1)_{l}$ Models & 18.178 & $18.496 \pm 2.160$ & $18.695 \pm 10.812$ & $18.875 \pm 35.510$ \\
\hline
\end{tabular}


The same plot ordering is mirrored in Figures 5.27 through 5.30 as in 5.23 through 5.30, except for an increase in integrated luminosity to $300 \mathrm{fb}^{-1}$. In these cases, the precision of the measurements are clear from the plots, and it is clear that generation universality can be determined in the models considered at all masses in the optimistic efficiency scenario, and for $M_{Z^{\prime}}<3000 \mathrm{GeV}$ in the pessimistic efficiency scenario.

Depending on the real efficiencies at the LHC detectors (ATLAS and CMS) and the model involved, it will be possible to distinguish whether the $Z^{\prime}$ couples universally potentially up to approximately $5 \%$ uncertainty for a $1000 \mathrm{GeV} Z^{\prime}$ and $10 \mathrm{fb}^{-1}$ integrated luminosity. As expected, the ability to determine the property of generation universality will be more difficult at higher $M_{\mathcal{Z}^{\prime}}$, and will require a greater number of events. However, it is possible to say that the level of non-generation universality in the Topcolor model presented will be distinguishable from that of the $S U(2)_{h} \times S U(2)_{l}$ and the $U(1)_{h} \times U(1)_{l}$ models, as presented (depending on the mixing parameter for each model) to $>95 \%$ confidence level at $1000 \mathrm{GeV}$ and to the $68 \%$ confidence level for a $2000 \mathrm{GeV} Z^{\prime}$, at optimistic efficiencies. In the pessimistic scenario, the statistics drop too rapidly for a $2000 \mathrm{GeV} Z^{\prime}$. 
$E 6 x$

E6 $\eta$

E6 $\psi$

LR Symmetric

Alt. LRSM

HARV

Sequential SM

Topcolor

Littlest Higgs

Simplest LH

Anomaly Free SLH

3-3-1 (2U 1D)

3-3-1 (1U 2D)

$\mathrm{SU}(2)_{\mathrm{h}} \times \mathrm{SU}(2)_{\text {, }}$

$\mathrm{U}(1)_{\mathrm{h}} \times \mathrm{U}(1)_{\mathrm{l}}$

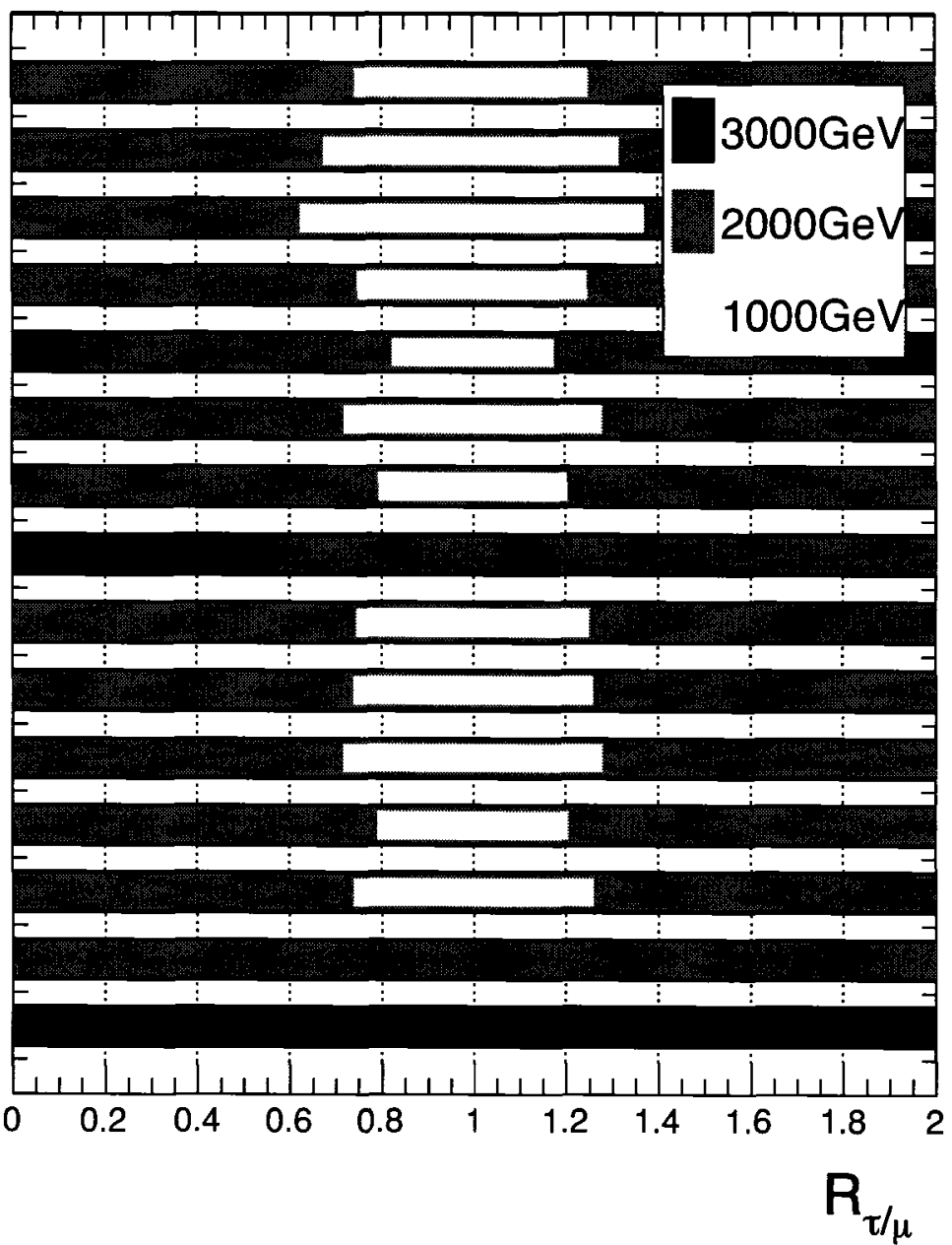

Figure 5.23: Plot of $1 \sigma$ uncertainty bounds for $R_{\tau / \mu}$ for each model at $10 \mathrm{fb}^{-1}$ integrated luminosity and pessimistic efficiencies $\left(\epsilon_{\tau}=0.01, \epsilon_{\mu}=0.96\right)$. The effect of mass of the $Z^{\prime}$ on uncertainty is shown. Range shown is selected to highlight models with generation universality. 
$E 6 \chi$

E6 $\eta$

E6 $\psi$

LR Symmetric

Alt. LRSM

HARV

Sequential SM

Topcolor

Littlest Higgs

Simplest LH

Anomaly Free SLH

3-3-1 (2U 1D)

3-3-1 (1U 2D)

$\mathrm{SU}(2)_{\mathrm{h}} \times \mathrm{SU}(2)_{\text {, }}$

$U(1)_{h} \times U(1)_{1}$

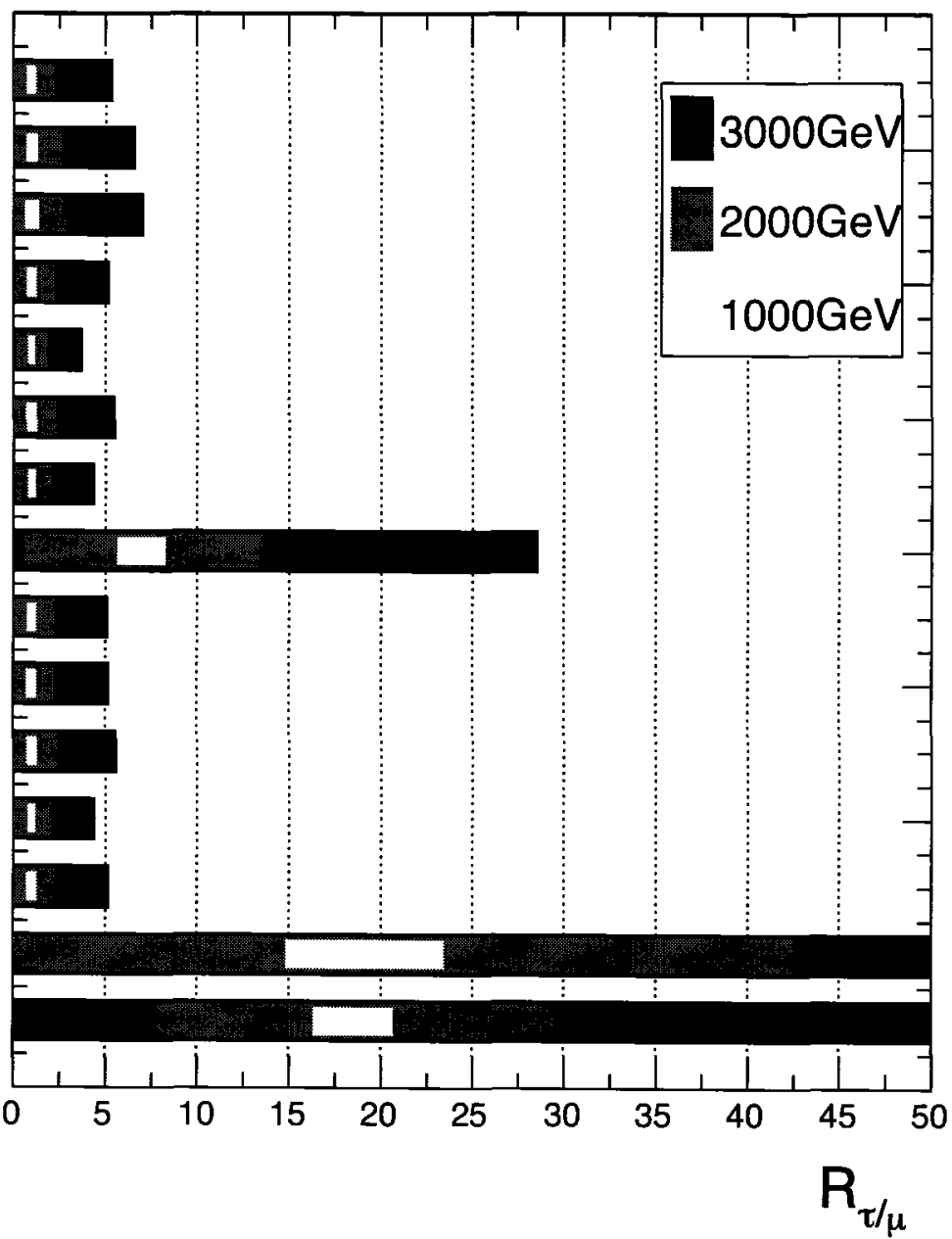

Figure 5.24: Plot of $1 \sigma$ uncertainty bounds for $R_{\tau / \mu}$ for each model at $10 \mathrm{fb}^{-1}$ integrated luminosity and pessimistic efficiencies $\left(\epsilon_{\tau}=0.01, \epsilon_{\mu}=0.96\right)$. The effect of mass of the $Z^{\prime}$ on uncertainty is shown. Range shown is selected to highlight models without generation universality. 
E6 $\chi$

E6 $\eta$

E6 $\psi$

LR Symmetric

Alt. LRSM

HARV

Sequential SM

Topcolor

Littlest Higgs

Simplest LH

Anomaly Free SLH

3-3-1 (2U 1D)

3-3-1 (1U 2D)

$\mathrm{SU}(2)_{\mathrm{h}} \times \mathrm{SU}(2)_{1}$

$\mathrm{U}(1)_{\mathrm{h}} \times \mathrm{U}(1)_{\mathrm{I}}$

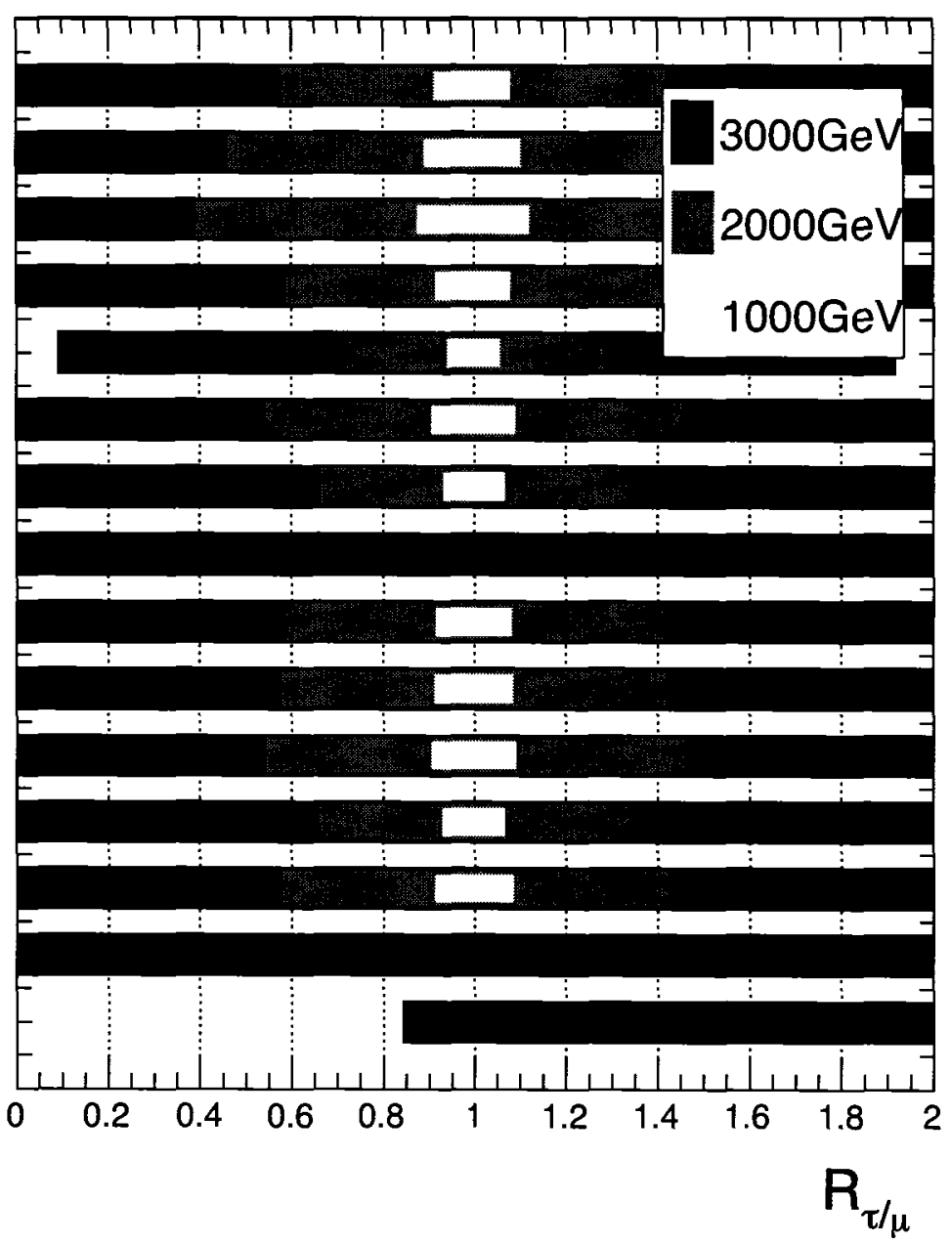

Figure 5.25: Plot of $1 \sigma$ uncertainty bounds for $R_{\tau / \mu}$ for each model at $10 \mathrm{fb}^{-1}$ integrated luminosity and optimistic efficiencies $\left(\epsilon_{\tau}=0.10, \epsilon_{\mu}=0.98\right)$. The effect of mass of the $Z^{\prime}$ on uncertainty is shown. Range shown is selected to highlight models with generation universality. 
E6 $\chi$

E6 $\eta$

E6 $\psi$

LR Symmetric

Alt. LRSM

HARV

Sequential SM

Topcolor

Littlest Higgs

Simplest LH

Anomaly Free SLH

3-3-1 (2U 1D)

3-3-1 (1U 2D)

$\mathrm{SU}(2)_{\mathrm{h}} \times \mathrm{SU}(2)_{\text {, }}$

$\mathrm{U}(1)_{\mathrm{h}} \times \mathrm{U}(1)_{\mathrm{l}}$

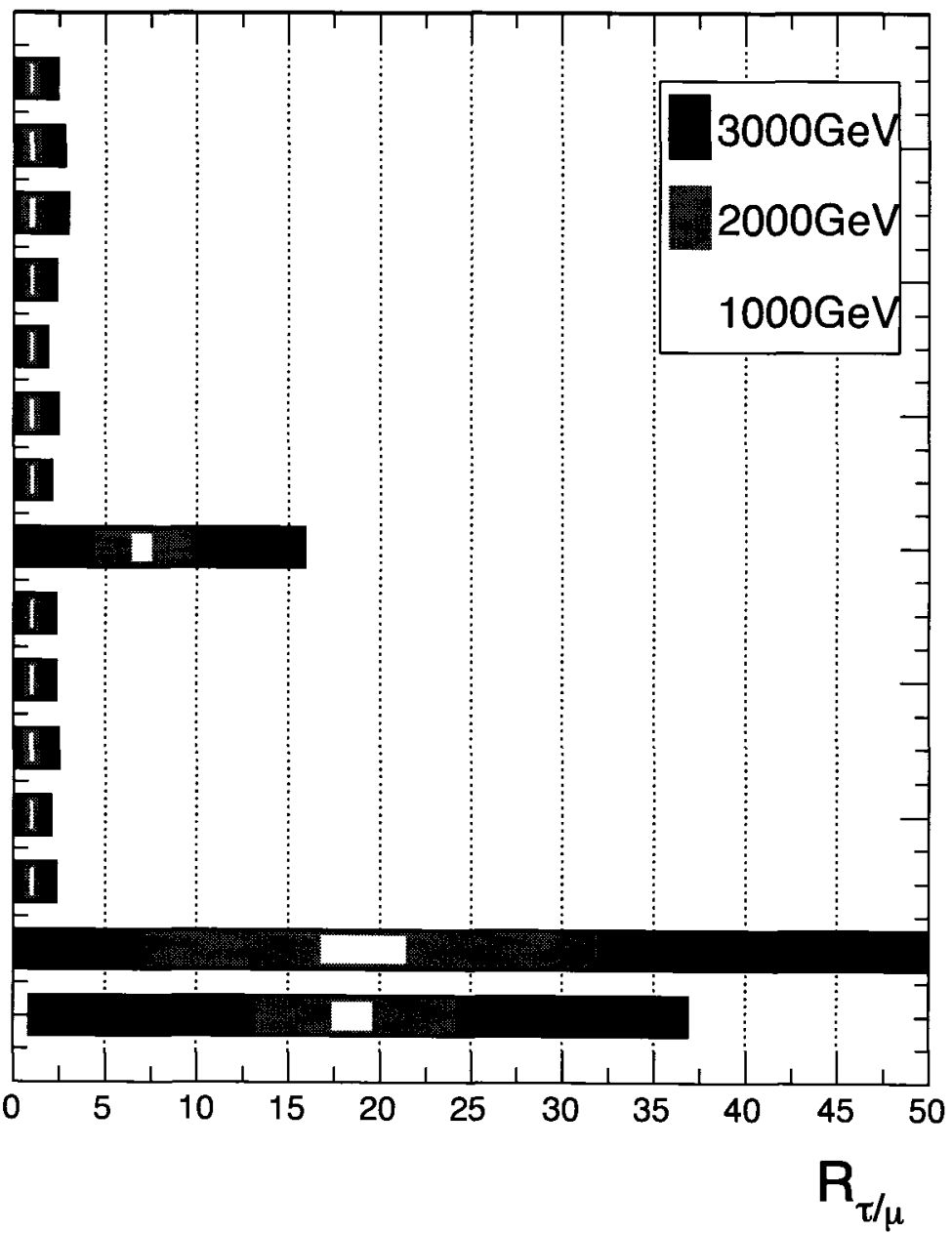

Figure 5.26: Plot of $1 \sigma$ uncertainty bounds for $R_{\tau / \mu}$ for each model at $10 \mathrm{fb}^{-1}$ integrated luminosity and optimistic efficiencies $\left(\epsilon_{\tau}=0.10, \epsilon_{\mu}=0.98\right)$. The effect of mass of the $Z^{\prime}$ on uncertainty is shown. Range shown is selected to highlight models without generation universality. 
$\mathrm{E} 6 \chi$

E6 $\eta$

E6 $\psi$

LR Symmetric

Alt. LRSM

HARV

Sequential SM

Topcolor

Littlest Higgs

Simplest LH

Anomaly Free SLH

3-3-1 (2U 1D)

3-3-1 (1U 2D)

$\mathrm{SU}(2)_{\mathrm{h}} \times \mathrm{SU}(2)_{1}$

$\mathrm{U}(1)_{\mathrm{h}} \times \mathrm{U}(1)_{\mathrm{l}}$

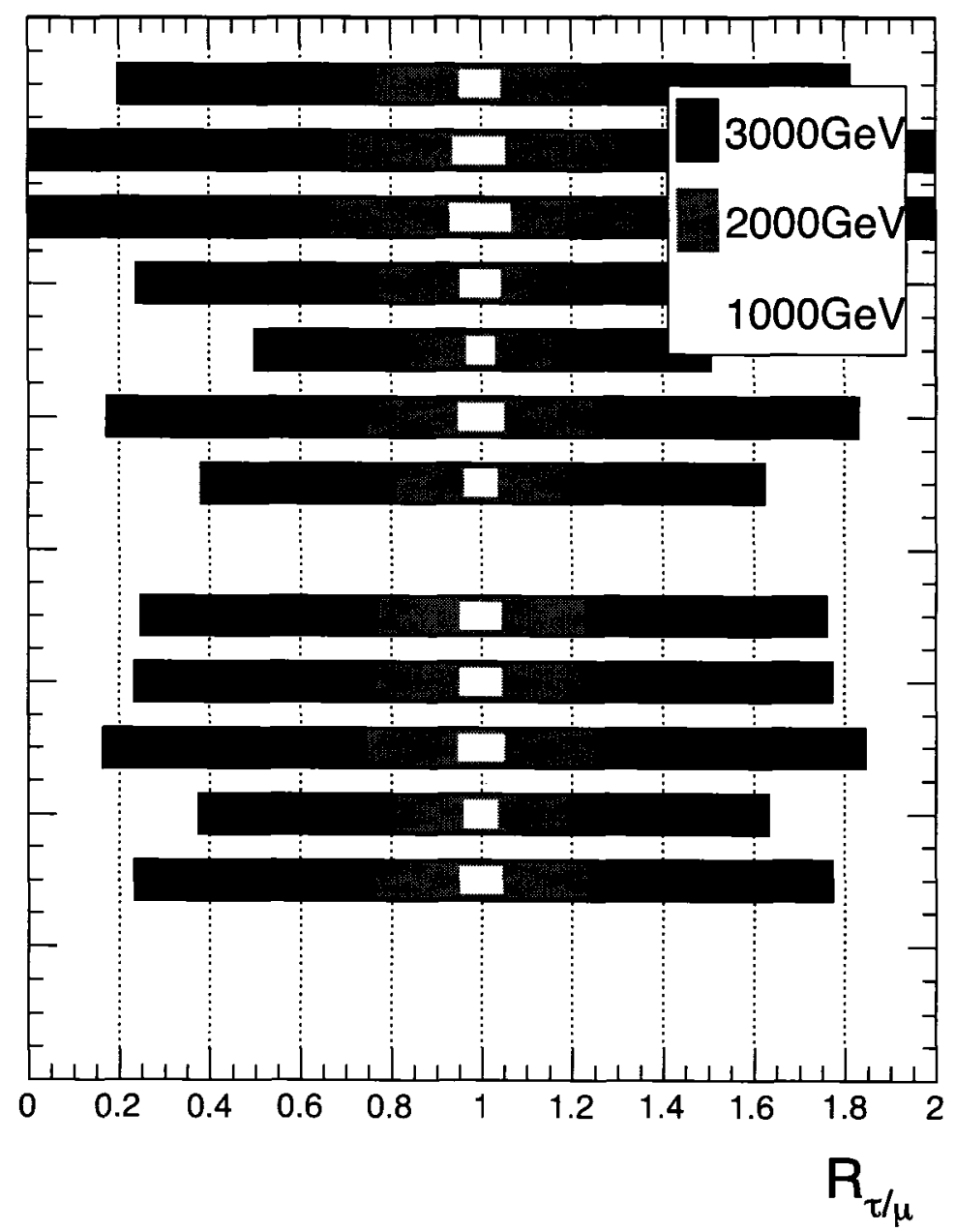

Figure 5.27: Plot of $1 \sigma$ uncertainty bounds for $R_{\tau / \mu}$ for each model at $300 \mathrm{fb}^{-1}$ integrated luminosity and pessimistic efficiencies $\left(\epsilon_{\tau}=0.01, \epsilon_{\mu}=0.96\right)$. The effect of mass of the $Z^{\prime}$ on uncertainty is shown. Range shown is selected to highlight models with generation universality. 
$E 6 \chi$

E6 $\eta$

E6 $\psi$

LR Symmetric

Alt. LRSM

HARV

Sequential SM

Topcolor

Littlest Higgs

Simplest LH

Anomaly Free SLH

3-3-1 (2U 1D)

3-3-1 (1U 2D)

$\mathrm{SU}(2)_{\mathrm{h}} \times \mathrm{SU}(2)_{1}$

$U(1)_{h} \times U(1)_{1}$

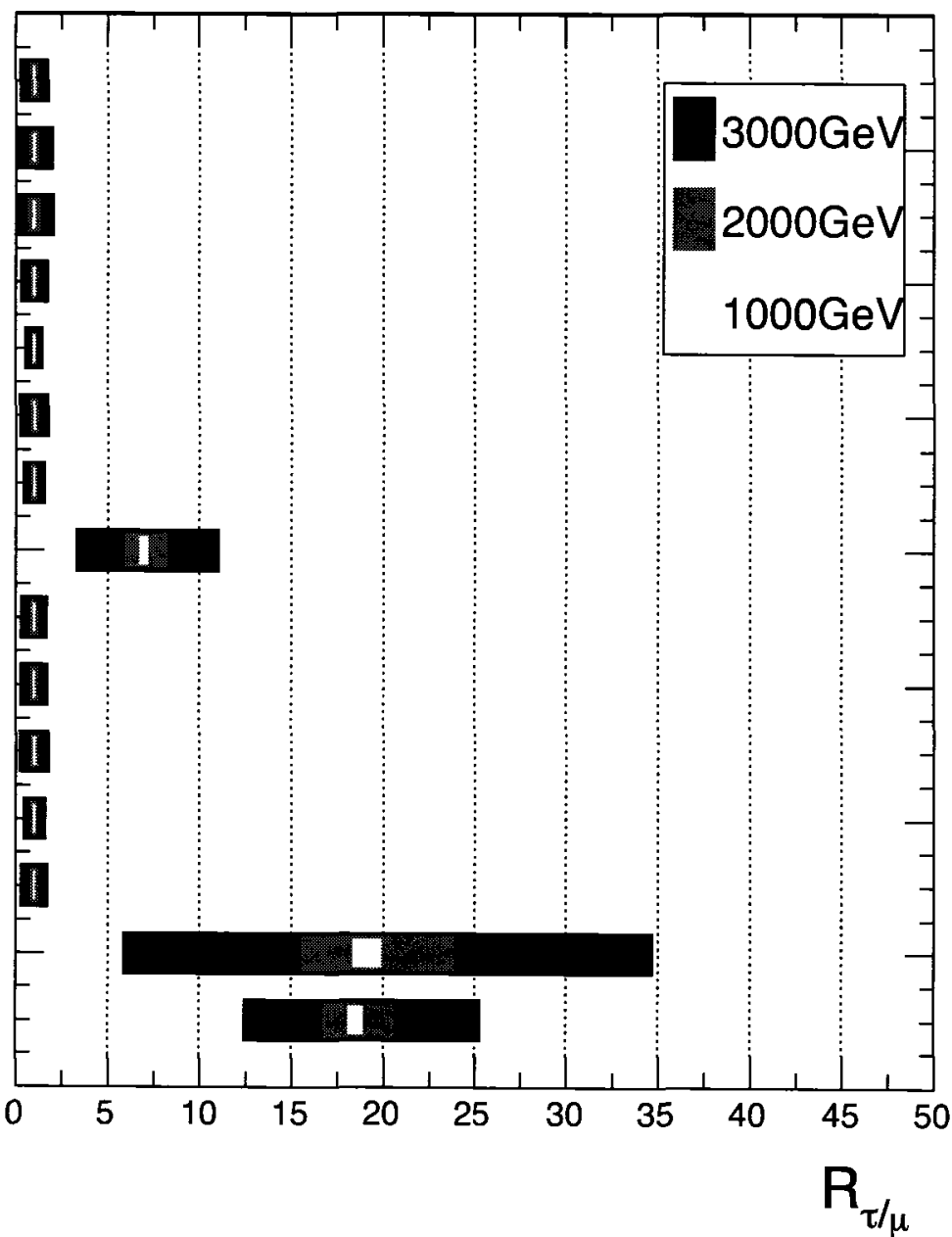

Figure 5.28: Plot of $1 \sigma$ uncertainty bounds for $R_{\tau / \mu}$ for each model at $300 \mathrm{fb}^{-1}$ integrated luminosity and pessimistic efficiencies $\left(\epsilon_{\tau}=0.01, \epsilon_{\mu}=0.96\right)$. The effect of mass of the $Z^{\prime}$ on uncertainty is shown. Range shown is selected to highlight models without generation universality. 
$\mathrm{E} 6 \chi$

E6 $\eta$

E6 $\psi$

LR Symmetric

Alt. LRSM

HARV

Sequential SM

Topcolor

Littlest Higgs

Simplest LH

Anomaly Free SLH

3-3-1 (2U 1D)

3-3-1 (1U 2D)

$\mathrm{SU}(2)_{\mathrm{h}} \times \mathrm{SU}(2)_{\text {, }}$

$\mathrm{U}(1)_{\mathrm{h}} \times \mathrm{U}(1)_{\mathrm{l}}$

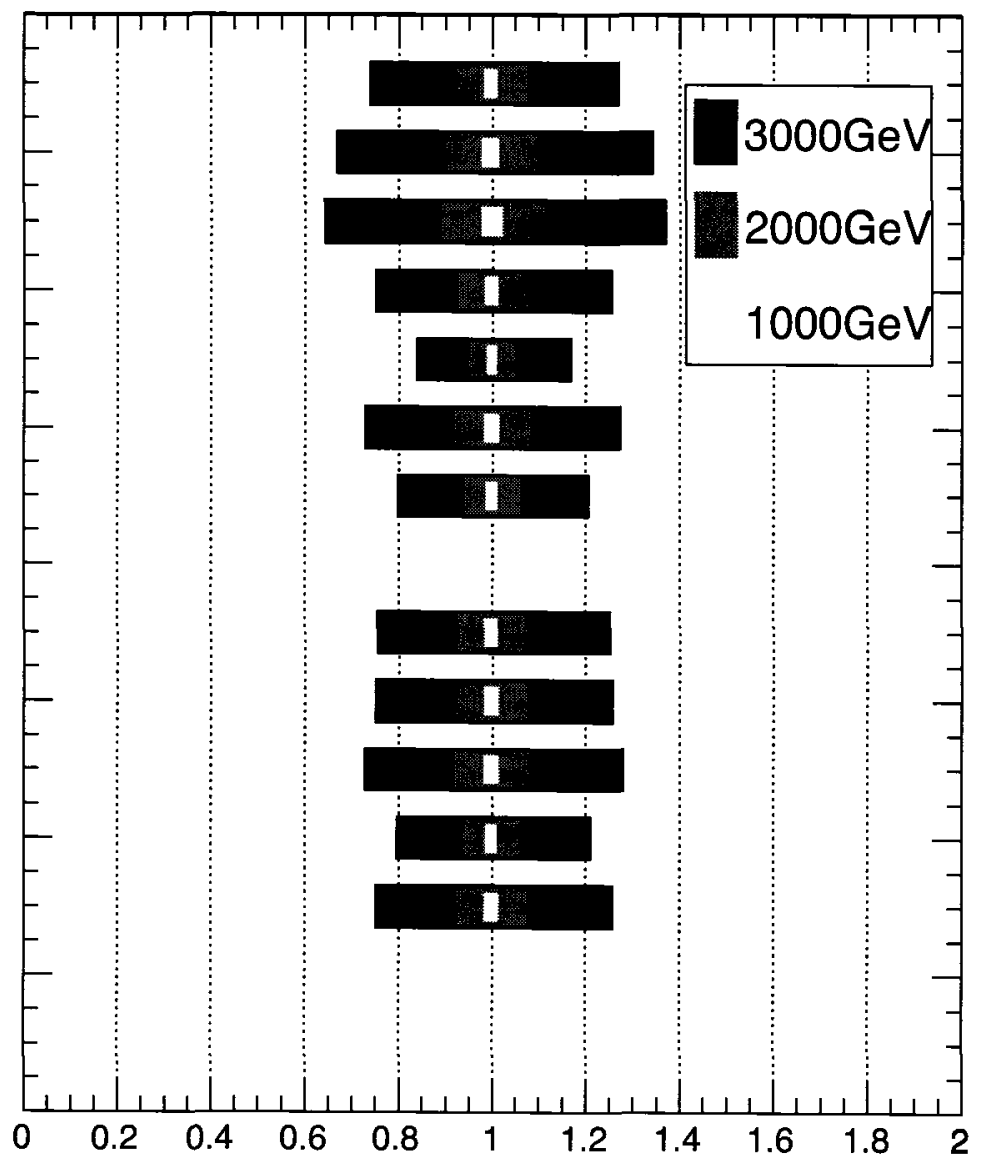

$\mathrm{R}$

Figure 5.29: Plot of $1 \sigma$ uncertainty bounds for $R_{\tau / \mu}$ for each model at $300 \mathrm{fb}^{-1}{ }_{\text {in- }}$ tegrated luminosity and optimistic efficiencies $\left(\epsilon_{\tau}=0.10, \epsilon_{\mu}=0.98\right)$. The effect of mass of the $Z^{\prime}$ on uncertainty is shown. Range shown is selected to highlight models with generation universality. 
$\mathrm{E} 6 \chi$

E6 $\eta$

E6 $\psi$

LR Symmetric

Alt. LRSM

HARV

Sequential SM

Topcolor

Littlest Higgs

Simplest LH

Anomaly Free SLH

3-3-1 (2U 1D)

3-3-1 (1U 2D)

$\mathrm{SU}(2)_{\mathrm{h}} \times \mathrm{SU}(2)_{\text {, }}$

$\mathrm{U}(1)_{\mathrm{h}} \times \mathrm{U}(1)_{\mathrm{l}}$

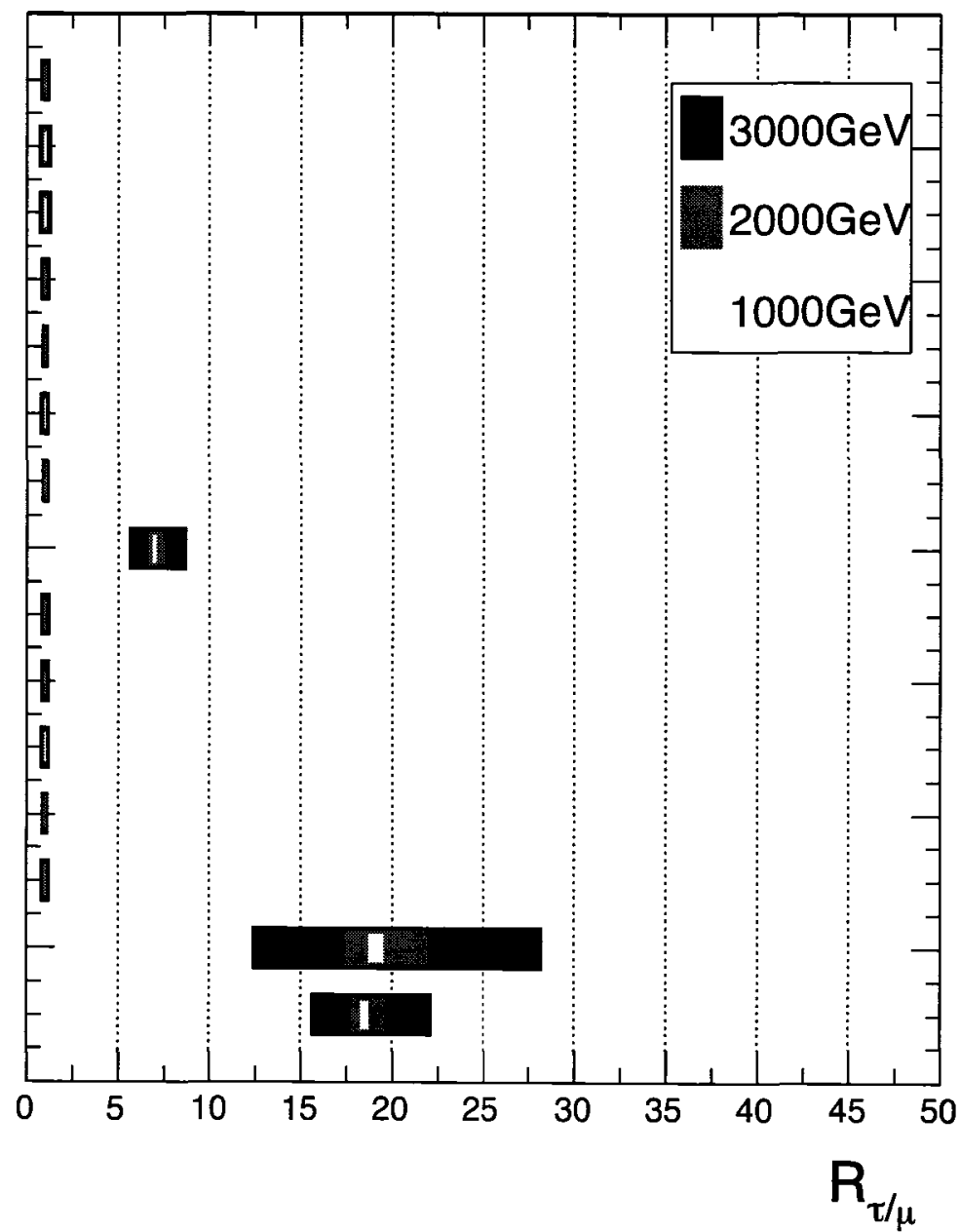

Figure 5.30: Plot of $1 \sigma$ uncertainty bounds for $R_{\tau / \mu}$ for each model at $300 \mathrm{fb}^{-1}$ integrated luminosity and optimistic efficiencies $\left(\epsilon_{\tau}=0.10, \epsilon_{\mu}=0.98\right)$. The effect of mass of the $Z^{\prime}$ on uncertainty is shown. Range shown is selected to highlight models without generation universality. 


\section{Chapter 6}

\section{Summary}

The discovery of a $Z^{\prime}$ may be one of the earliest successes of the LHC. In particular, a low mass $Z^{\prime}$ should be discoverable within the first few months of data collection unless the $Z^{\prime}$ is leptophobic or couples very weakly to the first two generations. The backgrounds at these energies and luminosities are negligible, and so even a small amount of signal will be a very distinct indicator that there is some BSM physics occurring.

Additionally, the ability to observe heavy quark final states presents an interesting and useful opportunity to analyze the nature of a new neutral gauge boson. By considering the ratio of the number of $t \bar{t}$ and $b \bar{b}$ events to $\mu^{+} \mu^{-}$events, the nature of the $Z^{\prime}$ becomes easily distinguishable. These measurements are highly dependent on the couplings to the individual particles and are independent of most free parameters for the models discussed. The exception occurs for models that prefer the lepton or quark sectors, and those with generation non-universality.

However, the complementary measurement of the ratio of third generation final 
states to first/second generation final states, within the same family, can further help distinguish between these cases, and elucidate the nature of generation universality for a general $Z^{\prime}$. In general, for low masses, the non-generation universal models can be distinguished from models with generation universality to the $95 \%$ confidence level at $10 \mathrm{fb}^{-1}$ luminosity. However, for higher masses, a significantly higher luminosity is required.

In each case, the tagging efficiencies for third generation final states was of primary importance, and presented the largest obstacle for reducing uncertainty. In general, efficiencies depend on kinematics and luminosities. Those presented were used as an average value and applied to show the viability of the measurement. A more detailed analysis, specifically a closer examination of top quark fragmentation using Pythia or some similar program, may be warranted to further study the viability of this specific measurement.

A study was presented of several techniques for distinguishing between models and determining whether the $Z^{\prime}$ arises from a model with generation dependent couplings. The techniques range in effectiveness, as some models provide similar results. The models chosen for this study comprised a list of interesting, and recent, possibilities. The study of models with preferential couplings to specific generations of fermions, as in the case of the tau to muon event ratio, presented an interesting opportunity for further analysis. Exploring the regions of mixing parameters is an important next step for these models.

I plan to continue the analysis of $Z^{\prime}$ final states, including a model independent study, and potentially a global analysis of all measurements and methods for distinguishing models, including as many models as possible - such as other variations of Topcolor and models of Extra Dimensions. Some models, such as the $\eta$ and $\chi$ are 
nearly indistinguishable in the analysis presented, as are others, and present specific challenges to examine. 


\section{References}

[1] F. Abe et al., Phys. Rev. Lett. 74, 2626 (1995).

[2] G. Weiglein et al., Phys. Rept. 426, 47 (2006).

[3] F. del Aguila, M. Cvetic, and P. Langacker, Phys. Rev. D48, 969 (1993).

[4] T. G. Rizzo, Phys. Rev. D47, 956 (1993).

[5] M. Cvetic and P. Langacker, Phys. Rev. D46, 14 (1992).

[6] D. Griffiths, Introduction to Elementary Particles (Wiley; New Ed edition, New York, 1987).

[7] W.-M. Yao et al., Journal of Physics G 33, 1+ (2006).

[8] S. Glashow, J. Iliopouos, and L. Maiani, Phys. Rev. D2, 1285 (1970).

[9] S. Weinberg, Phys. Rev. Lett 19, 1264 (1967).

[10] A. Salam, Elementary Particle Theory (Almquist and Wiksells, Stockholm, 1969).

[11] C. Quigg, Acta Phys. Polon. B30, 2145 (1999).

[12] J. Boger et al., Nucl. Instrum. Meth. A449, 172 (2000). 
[13] J. Hosaka et al., Phys. Rev. D74, 032002 (2006).

[14] R. Mohapatra, Unification and Supersymmetry (Springer, New York, 1986).

[15] M. Cvetic and S. Godfrey, arXiv:hep-ph/9504216 (1995).

[16] K. S. Babu, X.-G. He, and E. Ma, Phys. Rev. D36, 878 (1987).

[17] E. Ma, Phys. Rev. D36, 274 (1987).

[18] J. F. Gunion, A. Mendez, and F. I. Olness, Int. J. Mod. Phys. A2, 1085 (1987).

[19] T. G. Rizzo, Phys. Lett. B206, 133 (1988).

[20] J. L. Hewett and T. G. Rizzo, Phys. Rept. 183, 193 (1989).

[21] E. Witten, Nucl. Phys. B258, 75 (1985).

[22] E. Ramirez Barreto, Y. D. A. Coutinho, and J. Sa Borges, Eur. Phys. J. C50, 909 (2007).

[23] A. G. Dias, J. C. Montero, and V. Pleitez, Phys. Lett. B637, 85 (2006).

[24] R. M. Harris, C. T. Hill, and S. J. Parke, arXiv:hep-ph/9911288 (1999).

[25] C. T. Hill, Phys. Lett. B266, 419 (1991).

[26] C. T. Hill, Phys. Lett. B345, 483 (1995).

[27] T. Han, H. E. Logan, and L.-T. Wang, JHEP 01, 099 (2006).

[28] N. Arkani-Hamed, A. G. Cohen, and H. Georgi, Phys. Lett. B513, 232 (2001).

[29] N. Arkani-Hamed, A. G. Cohen, T. Gregoire, and J. G. Wacker, JHEP 08, 020 (2002). 
[30] N. Arkani-Hamed, A. G. Cohen, E. Katz, and A. E. Nelson, JHEP 07, 034 (2002).

[31] I. Low, W. Skiba, and D. Smith, Phys. Rev. D66, 072001 (2002).

[32] S. Chang and J. G. Wacker, Phys. Rev. D69, 035002 (2004).

[33] S. Chang, JHEP 12, 057 (2003).

[34] H. Georgi, E. E. Jenkins, and E. H. Simmons, Phys. Rev. Lett. 62, 2789 (1989).

[35] V. D. Barger and T. Rizzo, Phys. Rev. D41, 946 (1990).

[36] K. D. Lane and E. Eichten, Phys. Lett. B352, 382 (1995).

[37] K. D. Lane, Phys. Rev. D54, 2204 (1996).

[38] K. D. Lane, Phys. Lett. B433, 96 (1998).

[39] M. B. Popovic and E. H. Simmons, Phys. Rev. D58, 095007 (1998).

[40] R. S. Chivukula, E. H. Simmons, and J. Terning, Phys. Lett. B331, 383 (1994).

[41] R. S. Chivukula, E. H. Simmons, and J. Terning, Phys. Rev. D53, 5258 (1996).

[42] E. H. Simmons, Phys. Rev. D55, 5494 (1997).

[43] E. Malkawi, T. Tait, and C. P. Yuan, Phys. Lett. B385, 304 (1996).

[44] D. J. Muller and S. Nandi, arXiv:hep-ph/9610404 (1996).

[45] H.-J. He, T. Tait, and C. P. Yuan, Phys. Rev. D62, 011702 (2000).

[46] T. G. Rizzo, (2001).

[47] A. Abulencia et al., Phys. Rev. Lett. 96, 211801 (2006). 
[48] J. Erler and P. Langacker, Phys. Rev. Lett. 84, 212 (2000).

[49] M. Carena, A. Daleo, B. A. Dobrescu, and T. M. P. Tait, Phys. Rev. D70, 093009 (2004).

[50] B. A. Dobrescu and C. T. Hill, Phys. Rev. Lett. 81, 2634 (1998).

[51] R. S. Chivukula, B. A. Dobrescu, H. Georgi, and C. T. Hill, Phys. Rev. D59, 075003 (1999).

[52] D. E. Kaplan and M. Schmaltz, JHEP 10, 039 (2003).

[53] W. Skiba and J. Terning, Phys. Rev. D68, 075001 (2003).

[54] M. Schmaltz, JHEP 08, 056 (2004).

[55] O. C. W. Kong, J. Korean Phys. Soc. 45, S404 (2004).

[56] P. Abreu et al., Z. Phys. C65, 603 (1995).

[57] S. D. Drell and T.-M. Yan, Phys. Rev. Lett. 25, 316 (1970).

[58] S. Godfrey, J. L. Hewett, and T. G. Rizzo, Phys. Rev. D37, 643 (1988).

[59] A. Collaboration, Technical report, CERN/LHCC/99-15 (unpublished).

[60] C. Collaboration, Technical report, CERN/LHCC 2006-001 (unpublished).

[61] J. Pumplin et al., JHEP 07, 012 (2002).

[62] A. D. Martin, R. G. Roberts, W. J. Stirling, and R. S. Thorne, Eur. Phys. J. C28, 455 (2003).

[63] K. Agashe et al., arXiv:hep-ph/0612015 (2006). 
[64] B. Lillie, L. Randall, and L.-T. Wang, arXiv:hep-ph/0701166 (2007).

[65] V. Barger, T. Han, and D. G. E. Walker, arXiv:hep-ph/0612016 (2006).

[66] J. D. Anderson, M. H. Austern, and R. N. Cahn, Phys. Rev. Lett. 69, 25 (1992).

[67] O. Kortner, arXiv:0707.0905 [hep-ex] (2007).

[68] C. P. Hays, Nucl. Phys. Proc. Suppl. 169, 333 (2007).

[69] V. M. Abazov et al., Phys. Rev. D71, 072004 (2005).

[70] W. Kilian, LC-TOOL-2001-039 (2001). 